\title{
Economic Burden of Low-value Healthcare on Patients with Localized Prostate Cancer: Statistical \& Machine Learning Approaches
}

\author{
Ryan Fiano \\ rfiano@mix.wvu.edu
}

Follow this and additional works at: https://researchrepository.wvu.edu/etd

Part of the Health Services Research Commons

\section{Recommended Citation}

Fiano, Ryan, "Economic Burden of Low-value Healthcare on Patients with Localized Prostate Cancer: Statistical \& Machine Learning Approaches" (2021). Graduate Theses, Dissertations, and Problem Reports. 8118.

https://researchrepository.wvu.edu/etd/8118

This Dissertation is protected by copyright and/or related rights. It has been brought to you by the The Research Repository @ WVU with permission from the rights-holder(s). You are free to use this Dissertation in any way that is permitted by the copyright and related rights legislation that applies to your use. For other uses you must obtain permission from the rights-holder(s) directly, unless additional rights are indicated by a Creative Commons license in the record and/ or on the work itself. This Dissertation has been accepted for inclusion in WVU Graduate Theses, Dissertations, and Problem Reports collection by an authorized administrator of The Research Repository @ WVU.

For more information, please contact researchrepository@mail.wvu.edu. 
Economic Burden of Low-value Healthcare on Patients with Localized Prostate Cancer: Statistical \& Machine Learning Approaches

Ryan Fiano

Follow this and additional works at: https://researchrepository.wvu.edu/etd

Part of the Health Services Research Commons 


\title{
Economic Burden of Low-value Healthcare on Patients with Localized Prostate Cancer: Statistical \& Machine Learning Approaches
}

\author{
Ryan Fiano, MPH \\ Dissertation submitted \\ to the School of Medicine \\ at West Virginia University \\ in partial fulfillment of the requirements for the degree of \\ Doctor of Philosophy in \\ Clinical \& Translational Science \\ Usha Sambamoorthi, PhD, Co-chair \\ Traci LeMasters, PhD, Co-chair \\ Kim Innes, $\mathrm{PhD}$ \\ Malcolm D. Mattes, MD \\ Chan Shen, PhD \\ West Virginia Clinical \& Translational Science Institute \\ Morgantown, West Virginia \\ 2021
}

Keyword: Prostate Cancer, Patient Centered Care, Conservative Management, Healthcare Costs, Machine Learning, Patient Reported Experience Measures, Care Fragmentation

Copyright 2021 Ryan Fiano 


\title{
ABSTRACT \\ Economic Burden of Low-value Healthcare on Patients with Localized Prostate Cancer: Statistical \& Machine Learning Approaches
}

\author{
Ryan M. Fiano, MPH
}

Adults with incident localized prostate cancer represent a large, medically complex population at risk for lowvalue care. Evidence-based guidelines recommend conservative management (CM) for localized prostate cancer patients with multimorbidity and limited life expectancy, however, 2 in 3 still choose treatment. This dissertation pursued three Aims to address research gaps related to healthcare practices associated with significant morbidity and economic burden on older men with incident localized prostate cancer: 1) examine the leading predictors of low-value healthcare practice of prostate cancer treatment for low-risk prostate cancer; 2) assess the role of patient-reported experience with care on high-value prostate cancer management; and 3) estimate the association of high-value care on non-cancer related healthcare expenditures using machine learning and statistical approaches. In this study, 2 in 3 adults received low-value prostate cancer treatment. Multimorbidity and care fragmentation were among the leading predictors of low-value prostate cancer treatment and contrary to expectations, life expectancy was a weak predictor of treatment receipt. Social determinants of health were highly ranked predictors of treatment. Higher "timeliness of care" patient reported experience scores were associated with high-value CM use. Other forms of low-value care before incident prostate cancer diagnosis were associated with higher non-cancer related healthcare expenditures while highvalue CM was associated with lower costs. In summary, this dissertation highlights the negative effect of multimorbidity and care fragmentation on overtreatment, high-value care, and cost outcomes. Perceptions of timely care with healthcare providers and systems have significant impact on high-value CM use among older men with localized prostate cancer. This dissertation reports strong independent predictive associations of incremental low-value healthcare use before incident prostate cancer diagnosis to have significant increases on long-term non-cancer related costs. 


\section{DEDICATION}

For Haddie and Anderson. May you always have the courage, grit, and patience to pursue your dreams. 


\section{ACKNOWLEDGEMENTS}

I would like to first thank my mentor, Dr. Usha Sambamoorthi, for her commitment and dedication to me and this work. Dr. Usha is the most humble and intelligent I have encountered in my life. All her students recognize her incredible commitment as a mentor, and I am forever grateful for her patience and kindness.

Dr. Gregory Merrick has also made this dissertation possible. Dr. Merrick's unqualified support, encouragement, and constructive criticisms serve as a keystone in this work. Thank you, Doc!

To my colleagues Dr. JonDavid Pollock, Dr. Robert Galbreath, and Dr. Wayne Butler for both personal and professional guidance in this work and my life. You all have influenced my life in innumerable ways, and I thank you for your example.

I would like to thank my committee members Dr. Traci Lemasters, Dr. Kim Innes, Dr. Malcolm Mattes, and Dr. Chan Shen for their helpful input and patience throughout the completion of this dissertation.

To my CTSI and PSP classmates for being an overwhelming positive influence on my experience as a graduate student. I am grateful for the collegial atmosphere only possible among such selfless individuals. I wish you all the best in your promising futures.

I would like to thank Dr. Mark Olfert, Dr. Paul Chandler, Dr. Julie Lockman, and Dr. Joan Lakoski for facilitating a great academic experience.

I would like to thank my friends and family for their support. I would like to thank my wife, Amanda, and two children, Haddie and Anderson, who also endured the stress of completing a graduate degree. To my parents, Jacqueline and Victor Jr., my brothers Jason, Victor III, and Nicholas for their love, support, and patience. To my grandparents, Victor Fiano Sr. and Kathryn Fiano, for their unconditional love and jovial character that have formed the person I am today; you are missed but never forgotten. Together, we all share this accomplishment. 
List of Tables

\section{Table of Contents}

List of Figures

List of Appendices viii

List of Abbreviations

1 Introduction

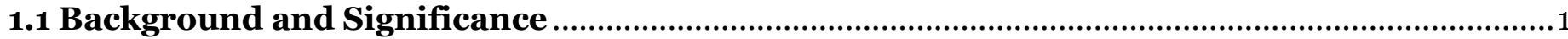

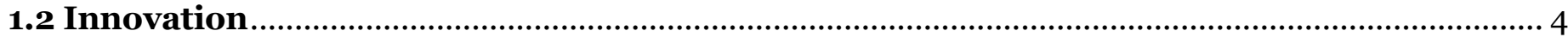

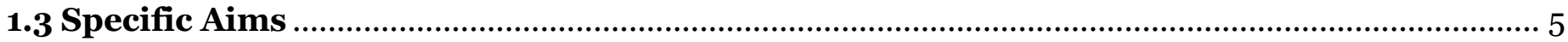

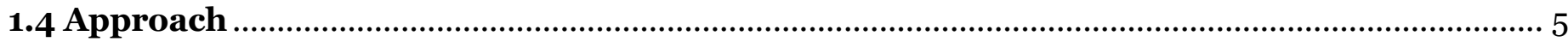

2 Prediction of Low-value Cancer Care among Older Men with Low-Risk Prostate Cancer: A Machine Learning Approach

2.1 Abstract

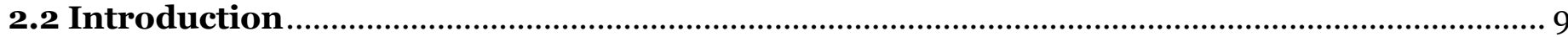

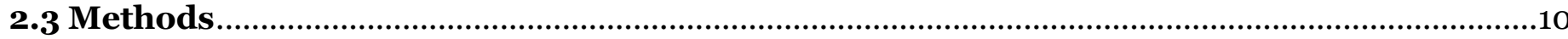

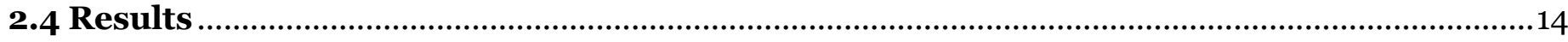

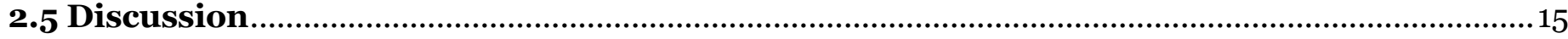

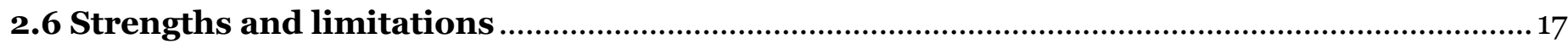

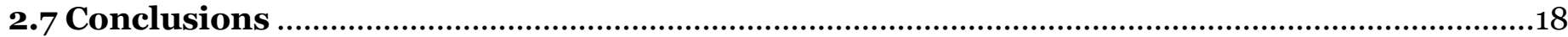

3 Associations of Multimorbidity and Patient-reported Experiences of Care with Conservative Management among Elderly Patients with Localized Prostate Cancer

3.1 Abstract

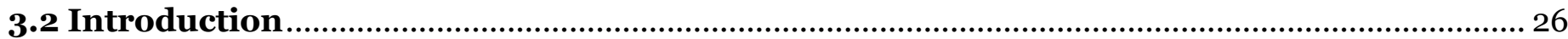

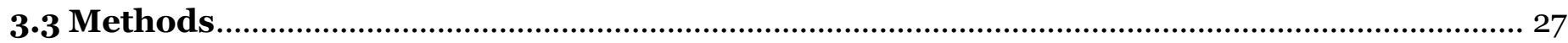

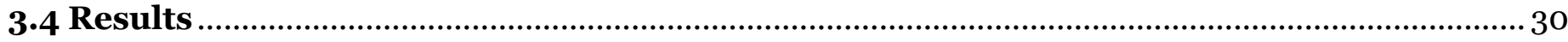

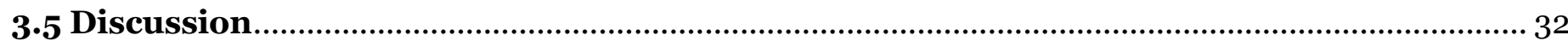

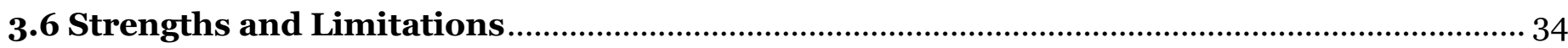

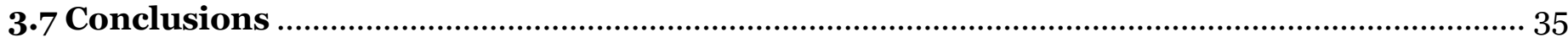

4 Healthcare Expenditures Associated with Low-value Care among Older Men with Incident Localized Prostate Cancer: Statistical and Machine Learning Approaches.

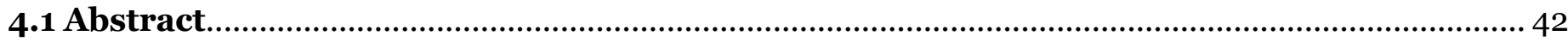

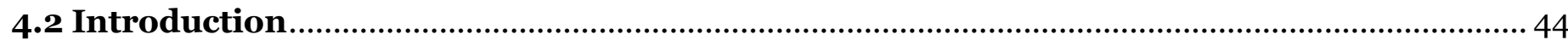

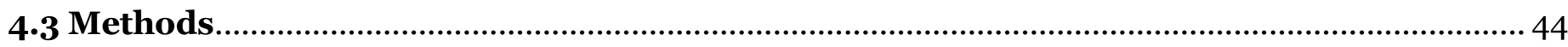

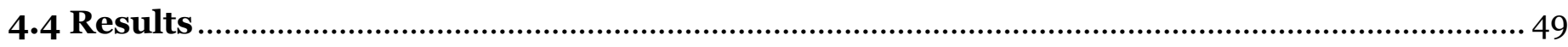

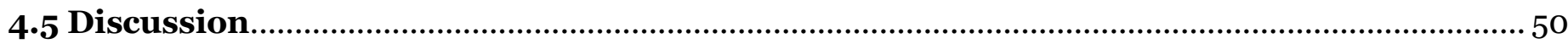

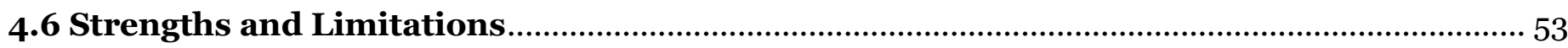

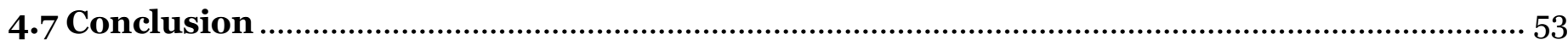

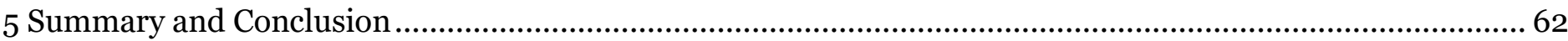




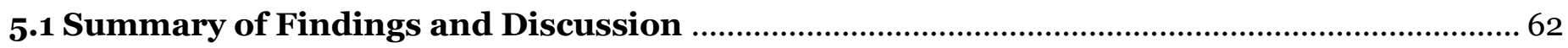

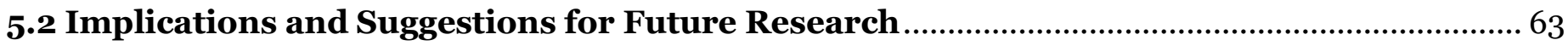

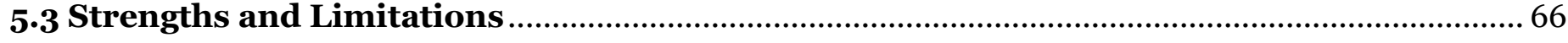

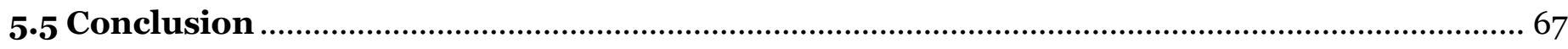

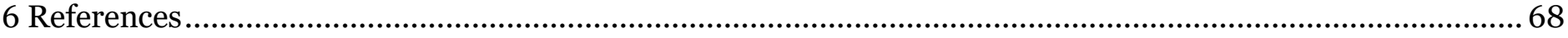

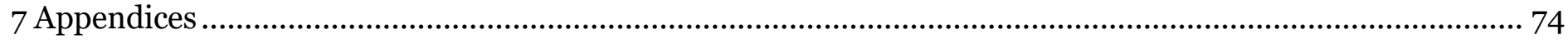




\section{List of Tables}

Table 2.1 Patient Characteristics by Treatment Use among Fee-for-Service Medicare Beneficiaries with Incident Low-risk Prostate Cancer using Linked SEER Cancer Registry, 2009-2014 ( $\mathrm{n}=13,870)$

Continued Table 2.1 Patient Characteristics by Treatment Use among Fee-for-Service Medicare Beneficiaries with Incident Low-risk Prostate Cancer using Linked SEER Cancer Registry, 2009-2014 ( $\mathrm{n}=13,870)$

Table 3.1 Patient Characteristics by Conservative Management among Fee-for-Service Medicare Beneficiaries with Incident Localized Prostate Cancer using Linked SEER Cancer Registry with MCAHPS, 2002-2013

$(n=496)$

Table 3.1 "continued"

Table 3.2 Multimorbidity and Patient Experiences by Conservative Management among Fee-for-Service Medicare Beneficiaries with Incident Localized Prostate Cancer using Linked SEER Cancer Registry with MCAHPS, 2002-2013

Table 3.3 Unadjusted (UOR), Adjusted Odds Ratios (AOR) and 95\% Confidence Intervals (CI) of Multimorbidity, Mental Health, Timeliness of Care, Low-risk Prostate Cancer, and Education on Likelihood of Conservative Management Use among Fee-for-Service Medicare Beneficiaries with Incident Localized Prostate Cancer using Linked SEER Cancer Registry with MCAHPS, 2002-2013 (N = 496).

Table 4.1 Patient Characteristics by Low-value Care Use among Fee-for-Service Medicare Beneficiaries with Incident Localized Prostate Cancer using Linked SEER Cancer Registry, 2005-2014 (n=75671)

continued Table 4.1

Table 4.2 Healthcare Expenditures by Low-value Care Use among Elderly Fee-for-Service Medicare Beneficiaries with Incident Localized Prostate Cancer SEER-Medicare, 2005-2014 (n=75671)

Table 4.3. Healthcare Expenditures by Conservative Management Use among Elderly Fee-for-Service Medicare Beneficiaries with Incident Localized Prostate Cancer SEER-Medicare, 2005-2014 (n=75671)

Table 4.4 Parameter estimates of PCCI categories from unadjusted and adjusted generalized linear models on 12-24 months healthcare expenditures among elderly Medicare fee-for-service beneficiaries with incident localized prostate cancer diagnosis using SEER-Medicare data, 2005-2015 ( $\mathrm{n}=75671)$. 


\section{List of Figures}

Figure 2.1 Feature Importance of Treatment Prediction among Fee-for-Service Medicare Beneficiaries with Incident Low-risk Prostate Cancer using SEER Cancer Registry, 2009-2014 (n=13870).

Figure 2.2 Positive and Negative Feature Relationships of Treatment Prediction among Fee-for-Service Medicare Beneficiaries with Incident Low-risk Prostate Cancer using SEER Cancer Registry, 2009-2014

$(\mathrm{n}=13870)$

Figure 2.3 SHAP Partial Dependence Plot of Top 4 Predictive Features of Treatment among Fee-for-Service Medicare Beneficiaries with Incident Low-risk Prostate Cancer using SEER Cancer Registry, 2009-2014

$(\mathrm{n}=13870)$

Figure 2.4 SHAP Partial Density Plot of Life Expectancy and Multimorbidity Predictive Features on Treatment among Fee-for-Service Medicare Beneficiaries with Incident Low-risk Prostate Cancer using SEER Cancer Registry, 2009-2014 ( $\mathrm{n}=13870)$

Figure 3.1 Cohort Selection and Exclusion

Figure 3.2 Adapted Competing Demands Framework

Figure 4.1 SHAP Feature Importance and Summary Plot of Low-value Care and Conservative Management Use on Non-Treatment Related Total Healthcare Expenditures among Fee-for-Service Medicare Beneficiaries with Incident Localized Prostate Cancer using Linked SEER Cancer Registry, 2005-2014 (n=75671)

Figure 4.2 SHAP Partial Density Plots of Selected Features on Non-Treatment Related Total Healthcare Expenditures among Fee-for-Service Medicare Beneficiaries with Incident Localized Prostate Cancer using Linked SEER Cancer Registry, 2005-2014 (n=75671) 


\section{List of Appendices}

Supplemental Figure 7.o Aim 1 Inclusion \& Exclusion Criteria

Supplemental Figure 7.1 Feature Importance of Treatment Prediction among Fee-for-Service Medicare Beneficiaries with Incident Low-risk Prostate Cancer using SEER Cancer Registry, 2009-2014 (n=13870) ......75

Appendix 7.2 Unadjusted and Adjusted Odds Ratios and 95\% Confidence Intervals (CI) of PCCI, Mental Health, Timeliness of Care, Low-risk Prostate Cancer, and Education on Conservative Management Use in among Fee-for-Service Medicare Beneficiaries with Incident Localized Prostate Cancer using Linked SEER Cancer Registry with MCAHPS, 2002-2013 ( $=496)$.

Appendix 7.3 Unadjusted and Adjusted Odds Ratios and 95\% Confidence Intervals of PCCI, Multimorbidity, Mental Health, Timeliness of Care, Low-risk Prostate Cancer, and Education on Conservative Management Use up to 24 Months after Incident Prostate Cancer among Fee-for-Service Medicare Beneficiaries using Linked SEER Cancer Registry with MCAHPS, 2002-2013 ( $=496)$

Appendix 7.4 Prostate cancer treatment codes used within 12 months of prostate cancer diagnosis to Identify

Conservative Management Use 78

Appendix 7.5 Patient Characteristics by Conservative Management among Fee-for-Service Medicare Beneficiaries with Incident Localized Prostate Cancer using Linked SEER Cancer Registry, 2005-2014 $(\mathrm{n}=75671)$

Appendix 7.6 Low-value Care by Conservative Management Use among Fee-for-Service Medicare Beneficiaries with Incident Localized Prostate Cancer using Linked SEER Cancer Registry, 2005-2014 (n=75671) . .81

Supplemental Figure 7.7 83

Appendix 7.8 Aim 1 python codes 84

Appendix 7.8 Aim 3 python codes 105 


\section{List of Abbreviations}

CM

PSA

PREM

LVC

ML

GLM

SDM

SHAP

PDP

PCCI

BBI

BETOS

HCPCS

SEER

MCAHPS

CCI

AOR

UOR

FFS
Conservative Management

Prostate Specific Antigen

Patient Reported Experience Measure(s)

Low Value Care

Machine Learning

Generalized Linear Model

Shared Decision Making

SHapley Additive exPlanations

Partial Density Plot

Prostate Cancer Comorbidity Index

Bice-Boxerman Continuity of care Index

Berenson-Eggers Type of Service codes

Healthcare Common Procedure Coding System

Surveillance Epidemiology, and End Results

Medicare Consumer Assessment of Healthcare Providers and Systems surveys

Charlson Comorbidity Index

Adjusted Odds Ratio

Unadjusted Odds Ratio

Fee-for-Service 


\section{CHAPTER 1}

\section{Introduction}

\subsection{Background and Significance}

\section{Epidemiology of localized prostate cancer}

Prostate cancer is the most frequently diagnosed malignancy and the 2nd leading cause of cancer death among men in the United States.(1) In the last several decades, prostate cancer-specific mortality has decreased significantly and future estimates project robust survivorship growth from 3.3 to 4.5 million by 2026.(2) Wide-spread use of prostate-specific antigen testing has increased detection of malignant and indolent cancer thus presenting evidence-based management challenges to address prostate cancer specific mortality of higher-, and avoid overtreatment of lower-, risk disease.(3)

\section{Localized, low-risk prostate cancer}

American Joint Commission on Cancer (AJCC) establishes a standard staging system using Tumor, Node, Metastasis (TNM) classifications for incident prostate cancer diagnoses; primary tumor (T), regional lymph node involvement (N), and distant metastasis (M) are assessed.(4) Localized prostate cancer, or cancer that is confined to the prostate, is classified as clinical stage T1 to T2a.(4) Risk stratification, broadly, includes clinical staging, Prostate Specific Antigen testing, and Gleason Score with 3 main risk groups: low-, intermediate-, and high- risk prostate cancer.(5) Low-risk prostate cancer is defined as T1 to T2a, Gleason 6, PSA $<10$ ng/mL, intermediate risk defined as T2b-T2c or Gleason 7 or higher or PSA 10-20 ng/mL, and high risk defined as T3a or Gleason 8 or higher or PSA > $20 \mathrm{ng} / \mathrm{mL} .(6)$

\section{Treatment selection for localized prostate cancer}

Older men with indolent localized prostate cancer and/or limited life expectancy are vulnerable to adverse effects of overtreatment. $(7,8)$ Overtreatment of localized prostate cancer is associated with negative health outcomes such as sexual, urinary, and rectal dysfunction including impotence, incontinence, and bowel irritation.(9-11) To avoid overtreatment morbidities, non-curative conservative management (CM) strategies have been established as an evidence-based option for adults with localized, low-risk cancer and/or limited life expectancy.(6,12) Clinical practice guidelines have recommended CM approaches for a decade.(13) More recently, providing curative treatment of low-risk prostate cancer or higher-risk disease among patients with 
less than 5 years of life expectancy without discussing CM is considered an indicator of sub-optimal healthcare quality.(12,14) Thus, the premise of CM is to avoid overtreatment-related adverse effects as localized malignancies pose little risk of prostate cancer-specific mortality.

\section{The Burden of Low-value Healthcare}

For decades, wasteful healthcare spending in the United States has not improved the cost, quality, and outcomes in comparison to other developed countries. Efforts to identify and address waste, as outlined in the Institute of Medicines (IOM) seminal work Crossing the Quality Chasm, have promoted a national effort to develop healthcare quality measurements to promote patient centered care; timely, well-coordinated care to inform healthcare decisions that reflect the patient's values.(15) To build on the IOM seminal work, The National Quality Task Force has redefined effective and patient-centered care as "appropriate, person-centered care”, thereby recognizing the adverse effects associated with inappropriate healthcare and affirming the need to understand chronic conditions management within the context of overall well-being.(16)

Low-value care, defined as inappropriate healthcare lacking a net clinical benefit to the patient, contributes to avoidable morbidities and excessive healthcare costs.(17) Low-value care is prevalent among elderly patients affecting 1 in 4 Medicare beneficiaries(18) and accounts for $\$ 75.7-\$ 101.2$ billion in annual healthcare costs. $(19,20)$ Use of low-value healthcare services among medically complex patients can lead to cascades of unnecessary down-stream care, cumulating costs as high as 10 times the original low-value healthcare service.(21)

To combat and eliminate low-value care, initiatives supported by the American Board of Internal Medicine Foundation have established the Choosing Wisely campaign to work with other professional societies to develop lists of low-value screenings, tests, and procedures.(22) Since the establishment of Choosing Wisely in 2012 study of low-value care has increased substantially. The National Quality Forum outlined opportunities for increasing requirements to "educate and engage" specific patient populations to reduce low-value care, while increasing patient-centered, high-value care.(16) However, few studies are oncology focused, with many examining the overuse of screening (i.e., breast and prostate cancer screening) versus more harmful impacts associated with overtreatment(23). Significant research gaps exist in the understanding how low-value care contributes to "down-stream" overtreatment and cost within specific clinical scenarios. 


\section{Patient-centered care}

Patients with incident localized prostate cancer represent a large, medically complex population at risk of low-value care in the form of overtreatment. Older patients with localized prostate cancer have high rates of multimorbidity, defined as more than 2 chronic conditions, and often experience an increase in care fragmentation at diagnosis.(24) Care fragmentation is known to present barriers to shared decision making and evidence-based recommendations among adults with multimorbidity, $(25,26)$ therefore adults with multimorbidity may be at increased risk for receiving overtreatment, low-value care, and/or increased downstream survivorship costs.

Shared decision making facilitates patient choice amid the risks and benefits of treatment and nontreatment options. Incident prostate cancer patients are challenged by multimorbidity and care fragmentation that influence perception of the healthcare system, potentially pushing them to choose an immediate cure(27) versus managing an additional chronic condition in a fragmented system. Fear of treatment regret, anxiety, and misunderstanding the risks and benefits of curative treatment within the context of cancer risk grouping and life expectancy are likely influenced by a patient's perception of care.(27)

The use of patient-reported experience measures can amplify the patient's perspective of the continuity of care among primary care and specialty clinicians. Patient reported experiences of care include domains such as physician communication, timely care, and perceptions of getting needed care and are increasingly used as quality measures by health plans, medical groups, and physician practices.(28) Positive patient-reported experience scores are associated with adherence to medical advice, improved clinical outcomes, and lower utilization of unnecessary health-care services.(15,29) Identifying specific measures of patient-reported experiences that facilitate CM use among patients with incident localized prostate cancer and multimorbidity is needed to promote evidence-based cancer care.(30) For example, in colorectal cancer populations, patientreported experiences with perceived timely care are associated with evidenced-based follow-up.(31) Understanding the relationship between patient-reported experiences of care on CM use can inform patientcentered care approaches by identifying gaps in timely care from the patient's perspective to improve adoption of CM use thereby reducing the adverse effects of overtreatment among older patients with multimorbidity and localized prostate cancer. 


\section{Economic burden of localized prostate cancer}

Many studies have examined comparative costs between different prostate cancer treatment modalities including CM.(32) CM use is known to reduce short-term treatment-related costs, however, long term costs associated with overtreatment or low-value care is not known. Use of low-value services prior to incident prostate cancer may be associated with increased costs throughout survivorship regardless of curative treatment or CM modalities.

\subsection{Innovation}

Using recent literature reviews(27,33-37) and healthcare initiative recommendations $(16,22)$, the research presented in this dissertation addresses significant research gaps as related to healthcare practices that provide limited clinical benefit, potentially harmful effects, and a significant economic burden on older men with incident localized prostate cancer. Specifically, we used novel machine learning techniques with nationally representative samples of older men with localized prostate cancer to answer research questions with both clinical and population relevance. We used interpretative model-agnostic approaches to understand the complex machine learning outputs with a focus on understanding predictors of localized prostate cancer treatment. We also used traditional statistical approaches to understand the impact of patient-reported outcomes on CM use and non-treatment related costs associated with the use or low-value care prior to incident localized prostate cancer diagnosis. We identified misalignment between prostate cancer specific, validated measures of life expectancy and use of guideline recommended care among localized prostate cancer patients. We also identified the predictive qualities of care fragmentation and use of low-value care on noncancer related healthcare expenditures during survivorship. The innovation of this work addresses the strategic objectives, research recommendations, and general research gaps as we identify novel factors, such as care fragmentation, low-value care use, and patient-reported outcomes, on high- and low-value treatment and costs outcomes among older adults with localized prostate cancer.

To highlight healthcare practices that provide limited clinical benefit, potentially harmful effects, and a significant economic burden among older men with incident localized prostate cancer, we pursue the following specific aims: 


\subsection{Specific Aims}

Aim 1: Use machine learning (ML) to identify leading predictors of cancer treatment within 12 months of diagnosis among older men with localized, low-risk prostate cancer.

- Quasi-hypothesis H1: Leading predictors of low-risk prostate cancer treatment will be age, multimorbidity, care fragmentation, and life expectancy.

Aim 2: Examine the associations of patient-reported experiences with providers and healthcare systems and multimorbidity on high-value care (CM use) among older men with localized prostate cancer.

- H2: Older men with multimorbidity and higher patient experience scores will be more likely to use CM compared to those without multimorbidity and lower patient experience scores, after adjusting for patient-, clinician-, and practice ecosystem factors.

Aim 3: Identify leading predictors and estimate the association of high- and low-value care use on non-cancer related healthcare expenditures among older men with localized prostate cancer.

- Quasi-hypothesis H3.1: Leading predictors of non-cancer related healthcare expenditures will be age, life expectancy, low- and high- value care.

- Quasi-hypothesis H3.2: Independent of treatment or conservative management use, adults using lowvalue care, versus no low-value care, will have higher non-treatment related healthcare costs 12-24 months after a localized prostate cancer diagnosis.

\subsection{Approach}

\section{Conceptual Framework}

Management of preexisting multimorbidity, care fragmentation, and the shared decision making process associated with choosing treatment or conservative management for incident prostate cancer requires the use of limited resources (i.e., time to manage chronic conditions and availability of health-care professionals and resources). Therefore, in this dissertation we used a competing demands model to conceptualize factors known to affect localized prostate cancer treatment selection within clinician, patient, and practice ecosystem domains.(33,38,39)

\section{Data Sources}

For aims 1 and 3 we used the Surveillance, Epidemiology, and End Results (SEER)-Medicare linked 
data files. SEER-Medicare data includes data on all incident prostate cancer cases occurring in 18 regions throughout the United States. Incident prostate cancer, prostate cancer specific clinical information (i.e., Gleason Score and Prostate-specific antigen values), and cancer staging information were derived from the SEER database. Medicare claims were derived from inpatient, outpatient, and other files linked to SEER.

For aim 2 we used a separate SEER with Medicare Consumer Assessment of Healthcare Providers \& Systems (MCAHPS®) survey linkages. MCAHPS, administered by the CMS, use standardized and validated questionnaires to collect information on patient-reported experiences with health-care providers.(40) MCAHPS collection methodologies use a weighted probability sampling procedure covering all the 50 US states, DC, and Puerto Rico, which are then linked to SEER patients. $(40,41)$

Area Health Resource File (AHRF) files were linked via MEDPAR FIPS state and county codes and used to calculate urologist densities.(42) Census files were linked to calculate county-level median income and college education.

We used both statistical and machine learning approaches in this dissertation. All model adjustments were made with factors known to influence treatment or conservative management selection. We used frequency and percentages (i.e., Chi-square tests and t-tests) to identify significant group differences by categorical variables and standard deviations for continuous variables. Generalized linear models (GLM) with log-link and gamma distribution were used to estimate key independent variable associations with healthcare cost. In aim 2, multivariable logistic regression was used to estimate the effect of patient-reported experience measures of care and healthcare providers on conservative management use.

ML methods can determine the most effective and parsimonious model/algorithm through classification of a binary target (i.e., yes or no dependent variable) via iterative learning. We used the XGBoost classifier, a decision-tree ensemble machine learning algorithm, to predict prostate cancer treatment or CM use among patients with low-risk prostate cancer diagnosis.(43)

Traditional statistical approaches use the entire data set and goodness of fit statistics for model specification.(44) Machine learning techniques utilize a training and test data set to estimate the predictive accuracy of a model. In ML models, original datasets were split (70\%/30\%) into training and test data. Next, predicted labels and probabilities, or a regression form such as log expenditures, were optimized on the 
training data via hyperparameter tuning using stratified 10-fold cross validation. Hyperparameters can adjust regularization through iterative tuning runs. Stratified cross validation describes the process of splitting the training data into identical "folds" that can be used as versions of "unseen" data during testing. Once the XGBoost algorithm is optimized on training data for accuracy, it is "tested" on the original 30\% split. We assessed predictive performance on the test set using precision, recall, accuracy, and Precision-Recall Receiver Operating Curve scores for machine learning models in aim 1. In aim 3, XGBoost regressors we used to assess prediction of log-healthcare costs and performance was assessed using $\mathrm{R}^{2}$ and negative mean squared error.

\section{Shapley Additive exPlanations}

To improve machine learning model interpretability, Shapley Additive exPlanations (SHAP) were used to visually summarize the contributions and associations of the features to the target variable. SHAP values represent the magnitude of effect from each feature on the model output by conditional expectation when the

feature is "hidden". Attributed changes induced on the model output are then averaged overall possible feature orderings in the data set (i.e. coalition) to provide the SHAP value.(45) Feature importance can be calculated by sorting absolute average SHAP values whereas summary plots sort average SHAP outputs with positive and negative variations on the $\mathrm{x}$-axis. Partial dependence plots (PDP) average SHAP values for a feature by a feature's unit value (for example, average age SHAP values by the feature age in years). Unlike traditional PDP, SHAP PDP shows dispersion around feature units reflecting the impact of all the other features in the model. Lastly, PDP interactions display the attribution effect of two features simultaneously. For aim 1 and 3, model SHAP outputs are log-odds and log-cost, respectively. 


\section{CHAPTER 2}

\section{Prediction of Low-value Cancer Care among Older Men with Low-Risk Prostate Cancer: A Machine Learning Approach}

\subsection{Abstract}

Background: Older men with incident prostate cancer are vulnerable to low-value prostate cancer treatment. Despite evidence-based support for conservative management (i.e., non-treatment), approximately 2 in 3 Medicare beneficiaries receive treatment for low-risk prostate cancer. Adults with multimorbidity who experience care fragmentation are vulnerable to departures from evidence-based medicine. A comprehensive analysis of clinical and non-clinical factors, such as life expectancy and care fragmentation, that may drive lowvalue prostate cancer treatment is lacking.

Objective: Use machine learning (ML) to identify leading predictors of cancer treatment within 12 months of diagnosis among older men with low-risk prostate cancer. Novel predictors included validated prostate-cancer specific life expectancy and care fragmentation.

Methods: In this retrospective cohort study we linked Surveillance, Epidemiology, and End Results cancer Registry (SEER), Medicare Claims, Census, and Area Health Resource files and included older men with incident low-risk prostate cancer from 2009 to $2014(n=13,870)$. We used claims data to identify treatment (Yes/No) in the first 12 months after diagnosis. We used the XGboost algorithm and SHapley Additive exPlanations (SHAP) to rank feature importance in treatment prediction.

Results: In our study cohort ( $\mathrm{n}=13,870), 66.9 \%$ of older adults received cancer treatment. Age, multimorbidity, care fragmentation, social support, and social determinants were leading predictors of cancer treatment (Accuracy=0.70, Precision=0.71, Recall=.92, Precision-Recall Area Under the Curve =0.78).

Relationships of college education, income, and care fragmentation on low-value cancer treatment were nonlinear and complex. Life expectancy was a weak predictor of prostate cancer treatment.

Conclusion: Our results suggest that non-clinical factors such as social determinants, care fragmentation, and social support are the most important predictors of treatment among older men diagnosed with low-risk prostate cancer. Despite a critical role in evidence-based treatment recommendations, life expectancy had limited impact on treatment selection. 


\subsection{Introduction}

Localized prostate cancer diagnoses are often indolent, representing a large potential source of lowvalue cancer care. Older men with low-risk prostate cancer and limited life expectancy risk significant urinary, erectile, and rectal morbidities without the benefit of significant improvement in prostate cancer specific mortality outcomes.(7,8,10) High-level evidence discourages treatment (3) of individuals with low-risk prostate cancer with less than 10 years of life expectancy or higher-risk disease with a life expectancy of 5 years or less.(6) Despite evidence-based support for CM in recent years, treatment for low-risk prostate cancer is highly prevalent, for example, among Medicare beneficiaries as a majority (58\%) still receive treatment as compared to integrated healthcare network cohorts (20\%).(46)

Decision to treat low-risk prostate cancer with radiation, surgery, cryotherapy, chemotherapy, or hormone therapy requires life expectancy estimation within the context of prostate cancer specific mortality and death from competing risks. Evidence-based guidelines recommend conservative management for low-risk prostate cancer and life expectancy of 10 or fewer years.(6) Examining the impact of life expectancy as a predictor is critical in assessing low-value treatment of low-risk prostate cancer, however, many methods used in population studies vary considerably and many lack validity in prostate cancer populations.(34)

Multimorbidity is another factor that may influence treatment of low-risk cancer. Over 60\% of older adults with incident prostate have multimorbidity defined 2 or more chronic conditions.(47),(48) Prior studies with SEER-Medicare data have shown that older men with 3 or more chronic conditions were significantly more likely to get treated for localized prostate cancer in adjusted multivariable models.(49)

Adults with multimorbidity who experience care fragmentation are vulnerable to departures from evidence-based medicine and poorer health outcomes.(26) Approximately 50\% of adults with multimorbidity already see 3 or more specialists (50) and increased care fragmentation is associated with diagnosis of incident prostate cancer.(24) Medically complex adults experiencing care fragmentation prior to diagnosis are likely to encounter additional barriers to shared-decision making process critical for understanding the risks and benefits of treatment. Shared decision-making engagement fosters trusting relationships that facilitate communication of evidence-based recommendations and is associated with reductions in both cancer and noncancer low-value care use.(27,51) 
Supervised ML models are increasingly used to predict healthcare outcomes, quality, and cost outcomes.(52) However, many studies investigating low-value prostate cancer care use only conventional statistical approaches. A comprehensive analysis of clinical and non-clinical factors, namely, valid life expectancy measures, multimorbidity, and care fragmentation, that drive low-value cancer treatment among adults with incident low-risk prostate cancer is lacking. Supervised ML can be used to identify the leading predictors and non-linear relationships among medically complex adults with higher probability of overtreatment.

Therefore, the objective of this study was to identify leading predictors of low-value cancer care among older men with low-risk prostate cancer using machine learning methods (ML). We used supervised ML models to identify leading predictors of low-value prostate cancer care by using nationally representative linked cancer registry-Medicare claims data incorporating a comprehensive list of patient, clinical, and practice ecosystem features.

\subsection{Methods}

\section{Study Design}

In this retrospective cohort study, the date of incident low-risk prostate cancer diagnosis was used as an index date and 12 months before diagnosis was used as the baseline period. We defined the treatment for lowrisk prostate cancer (radiation, surgery, chemo- and hormone- therapies) during a period of 12 months after diagnosis based on validated methods for claims data.(53)

\section{Study population}

Older adults (age $\geq 66$ years) diagnosed with low-risk prostate cancer during the study period (20092014) were included in the study population. Cancer diagnosis was identified using International Classification of Diseases for Oncology (ICD-9). Low-risk prostate cancer was defined as American Joint Committee on Cancer stage T2a or less, Gleason Score $\leq 6$, and a PSA test $\leq 10 \mathrm{ng} / \mathrm{mL} .(6)$

\section{Data sources}

This study used the Surveillance, Epidemiology, and End Results (SEER)-Medicare linked data files. SEER-Medicare data includes data on all incident prostate cancer cases occurring in 18 regions throughout the United States. Incident prostate cancer, prostate cancer specific clinical information (i.e., Gleason Score and 
Prostate-specific antigen values), and cancer staging information were derived from the SEER database.

Patient Medicare claims were derived from inpatient, outpatient, and other files linked to SEER.

Area Health Resource File (AHRF) files were linked via MEDPAR FIPS state and county codes and were used to calculate urologist densities.(42) Census files were linked to calculate county-level median income and college education (percentage with college degree).

\section{Study inclusion/exclusion criteria}

Adults with low-risk prostate cancer were included in the study if alive and continuously enrolled in Medicare Parts A and B throughout the baseline and follow-up periods (Appendix Supplemental Figure 7.7). Adults with missing cancer stage, PSA values, Gleason Scores, and/or those diagnosed with prostate cancer at autopsy were excluded.

\section{Target variable: Prostate cancer treatment (yes/no)}

Prostate cancer treatment was estimated within the first 12 months after diagnosis using a previously validated, claims-based algorithm and included radiation, surgery, and chemo-, cyro-, and/or hormonetherapy.(53)

\section{Key features}

Life expectancy

Prostate cancer comorbidity index (PCCI) is a weighted comorbidity index validated in prostate cancer patient populations to predict life expectancy.(54) Medical colleges, patient advocacy groups and evidencebased recommend informed decision-making in using conservative management or treatment for men with low-risk prostate cancer and a life expectancy of greater than 10 years.(6) We calculated PCCI during the baseline period to create predictive models based on validated life expectancy (10 year or greater life expectancy; $\mathrm{PCCI}<8)$ to reflect evidence-based recommendations. $(6,54)$ Categorical variables at clinically meaningful cut-offs are presented in Table 2.1 and were also included in sensitivity analyses for interpretative purposes.

\section{Multimorbidity}

The multimorbidity framework developed by the United States Department of Health and Human Services for guiding programs, practice, and policy guided the selection of chronic conditions as follows: 
arthritis, asthma, coronary artery disease, congestive heart failure, chronic kidney disease, chronic obstructive pulmonary disease, cardiac arrhythmias, acute myocardial infarction, dementia, diabetes, depression, hepatitis, hyperlipidemia, hypertension, human immunodeficiency virus, osteoporosis, substance abuse, schizophrenia, stroke, and anemia.(55) We defined multimorbidity presence of 3 or more chronic conditions.

\section{Care fragmentation}

We used a modified version of the Bice-Boxerman continuity of care index to calculate care fragmentation during the 12 month baseline period. $(56,57)$ The continuity of care index represents the concentration of visits per patient among health care providers based on visit number, proportion of encounters to each provider, and total number of visits. The BBI has been used to compare care fragmentation among cancer survivors and is highly correlated with other measures of care fragmentation.(58) We used physician specialty codes representing primary care, oncology, and various specialist visit encounters; where $n$ is the total number of visits, $n k$ is the total number of visits associated with a physician specialty, and $k$ the total number of physician specialty codes.(59) For example, multiple encounters with a single provider would result in a score of zero, however, multiple encounters among several health care providers would result in a score approaching 100. We included primary care, oncology, and many other specialty codes.

\section{Other features}

Patient factors included age, race, marital status, county-level median income and education (college graduation percent). Practice ecosystem included urologist/radiation oncologist density, diagnosis year, SEER region and Rural groups (Metro, Urban, Rural). All features were calculated in the 12 months before cancer diagnosis.

Propensity to seek care was also measured by two domains: the number of low-value care services as well as high-value care. Low-value healthcare services were operationalized using a claims-based algorithm representing Choosing Wisely campaign recommendations via procedure, diagnosis, hospitalization, and Berenson-Eggers Type of Service (BETOS) codes using previously published methods.(6o-62) We estimated the feature importance of individual low-value care procedures and summed those with an importance score greater than zero. We operationalized preventative services based on the National Commission on Prevention Priorities by the American Academy of Family Physicians as "high-value" care which included lipid and A1c 
screenings, influenza vaccinations, and primary care well visits. We used Healthcare Common Procedure Coding System (HCPCS) codes to identify preventative care.

All predictors were measured during the 12 month baseline period before incident low-risk prostate cancer diagnosis.

\section{Statistical Analysis}

Chi-square tests and t-tests were used to identify significant group differences in prostate cancer treat or CM use by categorical variables. All statistical tests were 2-sided with a $5 \%$ Type I error rate and completed in SAS (SAS Institute Inc., Cary, NC, USA. Version 9.4, using Windows 10).

\section{Machine learning}

ML methods can determine the most effective and parsimonious model/algorithm through classification of a binary target (i.e., yes or no dependent variable) via iterative learning. We used the XGBoost classifier, a decision-tree ensemble machine learning algorithm, to predict prostate cancer treatment or CM use among patients with low-risk prostate cancer diagnosis.(43)

First, our original dataset was split (70\%/30\%) into training and test data. Next, predicted labels and probabilities were optimized on the training data via hyperparameter tuning using repeated stratified 10-fold cross validation. We used hyperparameter tuning on the training data to optimize performance and avoid overfitting. Hyperparameter tuning parameters codes are available in Appendix Table 7.1.

Final model predictions were evaluated using the original "hold-out" test data. We assessed predictive performance on the test set using precision, recall, accuracy, and Precision-Recall Receiver Operating Curve scores. Machine learning analyses were conducted using Python 3.7 with open-source scikit-learn 0.21.3 wrapper interface for XGBoost classifier.

\section{Machine learning interpretation}

In the ML model, Shapley Additive exPlanations (SHAP) were used to visually summarize the contributions and associations of the features to the target variable. SHAP values represent the magnitude of effect from each feature on the model output by conditional expectation when the feature is "hidden". Attributed changes induced on the model output are then averaged overall possible feature orderings in the data set (i.e. coalition) to provide the SHAP value.(45) Feature importance can be calculated by sorting 
absolute average SHAP values whereas summary plots sort average SHAP outputs with positive and negative variations on the x-axis. Partial dependence plots (PDP) average SHAP values for a feature by a feature's unit value (for example, average age SHAP values by the feature age in years). Unlike traditional PDP, SHAP PDP shows dispersion around feature units reflecting the impact of all the other features in the model. Lastly, PDP interactions display the attribution effect of two features simultaneously. All model SHAP outputs are log-odds and are ranked in decending order.

TreeSHAP was used to generate SHAP values, feature importance, partial dependence plots, and PDP interactions with Python 3.7 and the SHAP package (0.29.2).

\subsection{Results}

\section{Descriptive cohort statistics}

The study cohort was predominantly non-Hispanic, white (83.3\%), marital status of married (69.1\%) with a median age of $72.0(\mathrm{Mdn}=71.1, \mathrm{SD}=4.46)$.

Overall, marital status of married, younger men in the age group 66-74 years, higher income, higher education level, lower physician quartiles, and adults residing in Northeastern SEER regions more frequently received treatment for low-risk prostate cancer (Table 2.1). In recent years, (2013 and 2014) mean age was lower among adults using treatment $(\mathrm{M}=71.3, \mathrm{SD}=4.05)$ versus no treatment $(\mathrm{M}=71.7, \mathrm{SD}=4.29 ; \mathrm{p}<0.001)$. Life expectancy did not significantly differ between adults using treatment versus no treatment, however, multimorbidity was significantly more frequent among treatment groups (Table 2.1). Preventative care, flu vaccinations, and primary care visits were significantly more frequent among treatment groups.

\section{Highest ranking predictors}

Marital status of married, men in the age group 66-74 years, care fragmentation, median income, and college education were the top 5 predictive features of low-risk prostate cancer treatment (Figure 2.1). Men who were married, aged 66-74 years, and experienced care fragmentation were more likely, and those with higher median income and college education were less likely to receive treatment (Figure 2.1). Northeast SEER region, diagnostic year 2010, multimorbidity, and west SEER region, more recent diagnostic year (2014 and 2013), were associated with higher and lower SHAP values (log-odds) of treatment prediction, respectively

(Figure 2.1). 
Figure 2.3 shows the complex individual variation among the top 4 predictive features. Overall, SHAP log-odds decreased with higher median income, however, substatntial individual-level variation in log-odds by distinct median income values was observed (Figure $2.3(\mathrm{~d})$ ).

\section{Lowest ranking predictors}

Life expectancy of 10 or more years (PCCI) and non-cancer low-value care use ranked 22nd and $27^{\text {th }}$, respectively in feature importance out of 29 total features (Figure 2.1). SHAP partial density plots illustrate variation among adults with life expectancies less than 10 years with SHAP log-odds of treatment (Figure 2.4). Hispanic, black, and white race were 23rd, 24th, and 25th ranked predictors, respectively (Appendix 7.1)

\subsection{Discussion}

In this first study using ML to predict treatment among older men with low-risk prostate cancer, we observed that 2 in 3 adults with low-risk prostate cancer received treatment. Other recent SEER-Medicare data studies also report high (57.9\%)(63), while integrated care network studies report low (21\%), rates of low-risk prostate cancer treatment among older men $(65+$ years $)$. High rates of low-value treatment are concerning as many men are likely to experience avoidable, negative health related quality of life and higher healthcare costs. For example, in the Prostate Cancer Intervention vs Observation Trial (PIVOT), a survival benefit to treatment versus conservative management was not significant after 10 years of follow-up. Treatment of low-risk prostate cancer confers significant risk of avoidable morbidities(10) and imposes a heavy economic burden of 1.2 billion dollars in annual cost(64). We speculate that treatment for low-risk prostate cancer may be high as older men experience adverse mental health effects associated with notification of higher PSA levels, pending biopsy results, and a prostate cancer diagnosis.(27) However, we observed that diagnosis year was a leading predictor of treatment, with adults diagnosed in later years (i.e. 2013 and 2014) less likely to receive treatment for lowrisk prostate cancer. Our findings revealing diagnostic year as a highly ranked predictive feature are consistent with published studies that show treatment of Medicare FFS adults with low-risk prostate cancer have decreased substantially in the last decade from $86 \%$ to $58 \%(63)$.

Care fragmentation was among the leading predictors of treatment for low-risk prostate cancer in this study. Adults experiencing care fragmentation and incident prostate cancer may be inclined to "take care of it" (27), as managing non-cancer conditions becomes more complex after a prostate cancer diagnosis.(65) In our 
adjusted machine learning models, care fragmentation prior to incident prostate cancer diagnosis was the 3 rd highest in SHAP feature importance, suggesting a strong associative prediction of low-value prostate cancer treatment. We also observed that older men with multimorbidity were more likely to receive treatment, consistent with findings of our recently published study indicating that multimorbidity was significantly and positively associated with likelihood of treatment among older men with localized prostate cancer.(49) Multimorbidity adds to the complexity to prostate cancer management decisions given that limited physician and patient resources often compete for management. Specialty healthcare (i.e., urologists and oncologists) need after an incident prostate cancer diagnosis among older men with multimorbidity is known to increase care fragmentation.(24) However, although multimorbidity was associated with higher odds of treatment use in the current study, interactions with care fragmentation were not informative.

Surprisingly, life expectancy was not associated with treatment receipt in this study. Evidence-based guidelines are based on risk group stratification (i.e., PSA, Gleason Score) and life expectancy. Although clinical assessment of life expectancy is a critical element to informed decision making for older adults with low-risk prostate cancer, our study findings suggest that treatment selection might not sufficiently take into consideration life expectancy.(6) We speculate that greater variation in low-value care use between organizations, versus between physicians, may reflect practice patterns associated with physician affiliation and organization management (i.e., compensation, practice guidelines).(66) Findings of other published studies using alternative methods of life expectancy estimation have been inconsistent, with investigations showing both positive (67) and negative relationships (68) between comorbidity burden and CM use in Medicare FFS populations.(49) Taken together, clinical and population differences in comorbidity life expectancy definitions are likely to account for these mixed findings. We speculate that discrepancies may be explained by the use of common use of comorbidity indexes that imprecisely measure life expectancy in prostate cancer populations and likely account for mixed findings. $(34,69)$

A noteworthy finding of the current study is the apparent role of social determinants (i.e., education and income) in predicting treatment of low-risk prostate cancer. The relationships were complex among individuals living in counties with varying levels of income and education. Socio-demographic factors, such as SEER region, income, college education, and marital status were highly ranked predictors of treatment in this 
and many other (27) investigations. Social determinants of health have also been used to accurately predict inpatient and emergency room utilization, demonstrating how social determinants can improve predictive ability of ML models.(70) We found race to be a low-ranking predictor of treatment among low-risk prostate cancer. Unequal access to care in racial minorities may result in poor management and/or overtreatment of low-risk prostate cancer as rates of low-risk prostate cancer treatment among racial groups vary between integrated and fee-for-service cohorts.(71,72) However, we found race to be a low-ranking predictor of treatment among low-risk prostate cancer in this study.

To our knowledge, this study is the first application of machine learning to estimate predictors of prostate cancer treatment among adults with low-risk prostate cancer. Unlike traditional statistical approaches, robust resampling and cross-validation techniques can be used to "train" machine learning algorithms to accurately predict healthcare utilization. More recently, model agnostic interpretations, such as SHAP used in this study, enable feature importance ranking and visualizations of individual-level contributions of patient, clinician, and practice ecosystem factors on target variables. Trade-offs between high performance and interpretability of complex "black-box" algorithms can be mitigated with SHAP feature importance ranking and data visualization. Our approach identified the top predictors of low-value treatment of low-risk prostate cancer and complex non-linear associations among features of high importance. Our results inform clinicians, payers, and policies makers regarding the predictive associations of features articulated in guideline recommendations, namely the lack of predictive importance of life expectancy, and the importance of social determinants, multimorbidity, and care fragmentation.

\subsection{Strengths and limitations}

Our study has several notable strengths. We used novel machine learning and model agnostic interpretations (SHAP) to predict and understand treatment use among older men with low-risk prostate cancer. We included validated measures of prostate cancer-specific life expectancy to identify older men with low-risk prostate cancer that could benefit from conservative management. Commonly used proxy measures of comorbidity burden are limited in estimating death risk from prostate cancer. We distinguished between comorbidity and life expectancy while previous studies have used comorbidity burden as a surrogate of life expectancy.(34) Using SHAP, we noted both average and individual level variations in CM use which suggests 
many older men could benefit from policies and/or interventions designed to reduce low-value prostate cancer treatment.

Our study must be interpreted within the context of several limitations. Foremost, the study is a claimsbased, retrospective analysis; therefore, we cannot establish causality and our results are subject to unobservable variable bias and selection bias of paid Medicare claims. To minimize the proportion of missed claims we limited analyses to continously enrolled in Medicare (A\&B, without HMO) throughout the study period. Second, our analysis may not be generaliable to commerical insurance beneficiaries as we only observed Medicare FFS beneficiairies. Third, social determinants of health (i.e., income, education) were not available at the individual level therefore county level measures were used. We were unable to estimate patient preference with SEER-Medicare data, and were thus unable to account for adults with longer life expectancies who prefer curative treatment as conservative management or active surveillance are evidence-based treatment options.

\subsection{Conclusions}

Using interpretable machine learning approaches provided evidence for several strong, modifiable predictors of low-value treatment of low-risk prostate cancer, including care fragmentation and social determinants of health. Healthcare policies could reduce low-value treatment by addressing fragmentation of care and management of multimorbidity. "Health in all policies" (73) that address upstream features such as income and education should be considered to reduce low-value prostate cancer care associated with significant adverse health related quality of life and cost burdens. Despite a decade's worth of evidence-based recommendations of observation for adults with low-risk prostate cancer and a life expectancy of more than 10 years, we observed life expectancy to be a low-ranked predictor of treatment. Physician- and patient- focused education may be needed to utilize more precise life expectancy estimates in shared decision making of treatment modalities of low-risk prostate cancer. 


\begin{tabular}{|c|c|c|c|c|c|c|c|}
\hline & & $\mathrm{CM}$ & & Treatr & & $\mathrm{X}^{2}$ & $p$-value \\
\hline & & $\mathrm{n}=4596$ & 33.1 & 9274 & 66.9 & & \\
\hline \multirow[t]{3}{*}{ Multimorbidity } & & & & & & 66.697 & $<0.001$ \\
\hline & o-2 MM & 2157 & 37.0 & 3678 & 63.0 & & \\
\hline & $>2 \mathrm{MM}$ & 2439 & 30.4 & 5596 & 69.6 & & \\
\hline \multirow[t]{4}{*}{ Life expectancy } & & & & & & 5.091 & 0.078 \\
\hline & $10+$ years & 3555 & 33.5 & 7057 & 66.5 & & \\
\hline & 5-10 years & 714 & 31.1 & 1580 & 68.9 & & \\
\hline & $<5 \mathrm{LE}$ & 327 & 33.9 & 637 & 66.1 & & \\
\hline \multirow[t]{3}{*}{ Age group } & & & & & & 113.674 & $<0.001$ \\
\hline & 66-74 & 3287 & 30.8 & 7384 & 69.2 & & \\
\hline & 75 and above & 1309 & 40.9 & 1890 & 59.1 & & \\
\hline \multirow[t]{6}{*}{ Race } & & & & & & 18.128 & $<0.001$ \\
\hline & White & 3796 & 32.8 & 7762 & 67.2 & & \\
\hline & Black & 497 & 35.4 & 908 & 64.6 & & \\
\hline & Hispanic & 48 & 22.6 & 164 & 77.4 & & \\
\hline & Others & 231 & 36.7 & 398 & 63.3 & & \\
\hline & Unknown & 24 & 36.4 & 42 & 63.6 & & \\
\hline \multirow[t]{5}{*}{ Marital status } & & & & & & \begin{tabular}{|l|}
209.023 \\
\end{tabular} & $<0.001$ \\
\hline & Unmarried & 318 & 35.5 & 577 & 64.5 & & \\
\hline & Married & 2843 & 29.7 & 6742 & 70.3 & & \\
\hline & Sep/div/wid & 545 & 37.6 & 903 & 62.4 & & \\
\hline & Unknown & 890 & 45.8 & 1052 & 54.2 & & \\
\hline \multirow[t]{5}{*}{ Income quartile } & & & & & & 17.902 & $<0.001$ \\
\hline & $\$ 2,512-43,709$ & 997 & 30.4 & 2286 & 69.6 & & \\
\hline & $\$ 43,711-57,34$ & 1058 & 32.6 & 2185 & 67.4 & & \\
\hline & $\$ 57,350-76,87$ & 1142 & 34.6 & 2156 & 65.4 & & \\
\hline & $\begin{array}{l}76,888- \\
250,0\end{array}$ & 1283 & 34.4 & 2442 & 65.6 & & \\
\hline \multirow[t]{5}{*}{$\begin{array}{l}\text { Education } \\
\text { quartile }\end{array}$} & & & & & & 8.371 & 0.039 \\
\hline & $0.00-24.0$ & 1192 & 33.5 & 2370 & 66.5 & & \\
\hline & $24-29.0$ & 1066 & 31.6 & 2306 & 68.4 & & \\
\hline & 29.0-34.1 & 1088 & 32.4 & 2269 & 67.6 & & \\
\hline & 34.1-100 & 1135 & 34.8 & 2130 & 65.2 & & \\
\hline \multirow[t]{5}{*}{ Rural group } & & & & & & 3.470 & 0.325 \\
\hline & Metro & 3925 & 33.4 & 7824 & 66.6 & & \\
\hline & Urban & 587 & 32.0 & 1248 & 68.0 & & \\
\hline & Rural & $* *$ & $* *$ & $* *$ & $* *$ & & \\
\hline & \begin{tabular}{|l} 
Unknown \\
\end{tabular} & $* *$ & $* *$ & *** & $* *$ & & \\
\hline
\end{tabular}

Based on 13870 older (age $\geq 66$ years) Fee-for-Service Medicare beneficiaries, with continuous enrollment in Medicare Part A \& Part B, diagnosed with incident low-risk prostate cancer (Gleason 6, PSA <10, Stage $\leq$ T2a) between 2009 and 2014.

Age group included 66-74 and 75 and over categories. SEER= Surveillance, Epidemiology, and End Results cancer Registry.

$* *$ Cells suppressed in accordance with Data Usage Agreement due to $<11$ cell count.Bold values denote statistical significance at the P-value $<.05$ level. 


\begin{tabular}{|c|c|c|c|c|c|c|c|}
\hline \multicolumn{8}{|c|}{$\begin{array}{l}\text { Continued Table 2.1 Patient Characteristics by Treatment Use among Fee-for-Service } \\
\text { Medicare Beneficiaries with Incident Low-risk Prostate Cancer using Linked SEER Cancer } \\
\text { Registry, 2009-2014 }(\mathrm{n}=13,870)\end{array}$} \\
\hline \multirow[t]{3}{*}{ Metro group } & & & & & & 5.253 & 0.072 \\
\hline & Metro county & 3940 & 33.5 & 7827 & 66.5 & & \\
\hline & Non-metro county & 648 & 31.1 & 1436 & 68.9 & & \\
\hline \multirow[t]{5}{*}{ SEER Region } & & & & & & 106.457 & $<0.001$ \\
\hline & Northeast & 838 & 27.8 & 2171 & 72.2 & & \\
\hline & South & 1171 & 30.5 & 2666 & 69.5 & & \\
\hline & North Central & 433 & 32.6 & 896 & 67.4 & & \\
\hline & West & 2154 & 37.8 & 3541 & 62.2 & & \\
\hline \multirow[t]{5}{*}{$\begin{array}{l}\text { Radiation } \\
\text { oncology quartile }\end{array}$} & & & & & & 14.578 & 0.002 \\
\hline & $0-0.44$ & 1128 & 31.2 & 2485 & 68.8 & & \\
\hline & $0.44-0.99$ & 1207 & 32.3 & 2527 & 67.7 & & \\
\hline & $0.99-1.47$ & 1085 & 35.2 & 1997 & 64.8 & & \\
\hline & $1.48-22.0$ & 1168 & 34.1 & 2254 & 65.9 & & \\
\hline \multirow[t]{5}{*}{ Urologist quartile } & & & & & & 17.438 & $<0.001$ \\
\hline & $0-1.25$ & 1117 & 30.4 & 2562 & 69.6 & & \\
\hline & $1.25-2.38$ & 1255 & 34.2 & 2411 & 65.8 & & \\
\hline & $2.38-3.22$ & 1039 & 34.3 & 1990 & 65.7 & & \\
\hline & $3.23-28.4$ & 1177 & 33.9 & 2300 & 66.1 & & \\
\hline \multirow[t]{3}{*}{$\begin{array}{l}\text { Preventative A1c } \\
\text { screening }\end{array}$} & & & & & & 2.238 & 0.135 \\
\hline & Yes & 650 & 34.6 & 1226 & 65.4 & & \\
\hline & No & 3946 & 32.9 & 8048 & 67.1 & & \\
\hline \multirow[t]{3}{*}{$\begin{array}{l}\text { Preventative Flu } \\
\text { vaccination }\end{array}$} & & & & & & 57.509 & $<0.001$ \\
\hline & Yes & 2113 & 30.1 & 4898 & 69.9 & & \\
\hline & No & 2483 & 36.2 & 4376 & 63.8 & & \\
\hline \multirow[t]{3}{*}{$\begin{array}{l}\text { Preventative lipid } \\
\text { screening }\end{array}$} & & & & & & 0.002 & 0.967 \\
\hline & Yes & 1283 & 33.1 & 2592 & 66.9 & & \\
\hline & No & 3313 & 33.1 & 6682 & 66.9 & & \\
\hline \multirow[t]{3}{*}{$\begin{array}{l}\text { Primary care } \\
\text { physician visit }\end{array}$} & & & & & & 44.881 & $<0.001$ \\
\hline & Yes & 3618 & 30.6 & 8205 & 69.4 & & \\
\hline & No & 628 & 38.9 & 988 & 61.1 & & \\
\hline
\end{tabular}

Based on 13870 older (age $\geq 66$ years) Fee-for-Service Medicare beneficiaries, with continuous enrollment in Medicare Part A \& Part B, diagnosed with incident low-risk prostate cancer (Gleason 6, PSA <10, Stage <T2a) between 2009 and 2014.

Age group included 66-74 and 75 and over categories.

SEER= Surveillance, Epidemiology, and End Results cancer Registry.

Bold values denote statistical significance at the P-value $<.05$ level. 
Figure 2.1 Feature Importance of Treatment Prediction using SHAP values among Fee-for-Service Medicare Beneficiaries with Incident Low-risk Prostate Cancer using SEER Cancer Registry, 2009-2014 (n=13870)

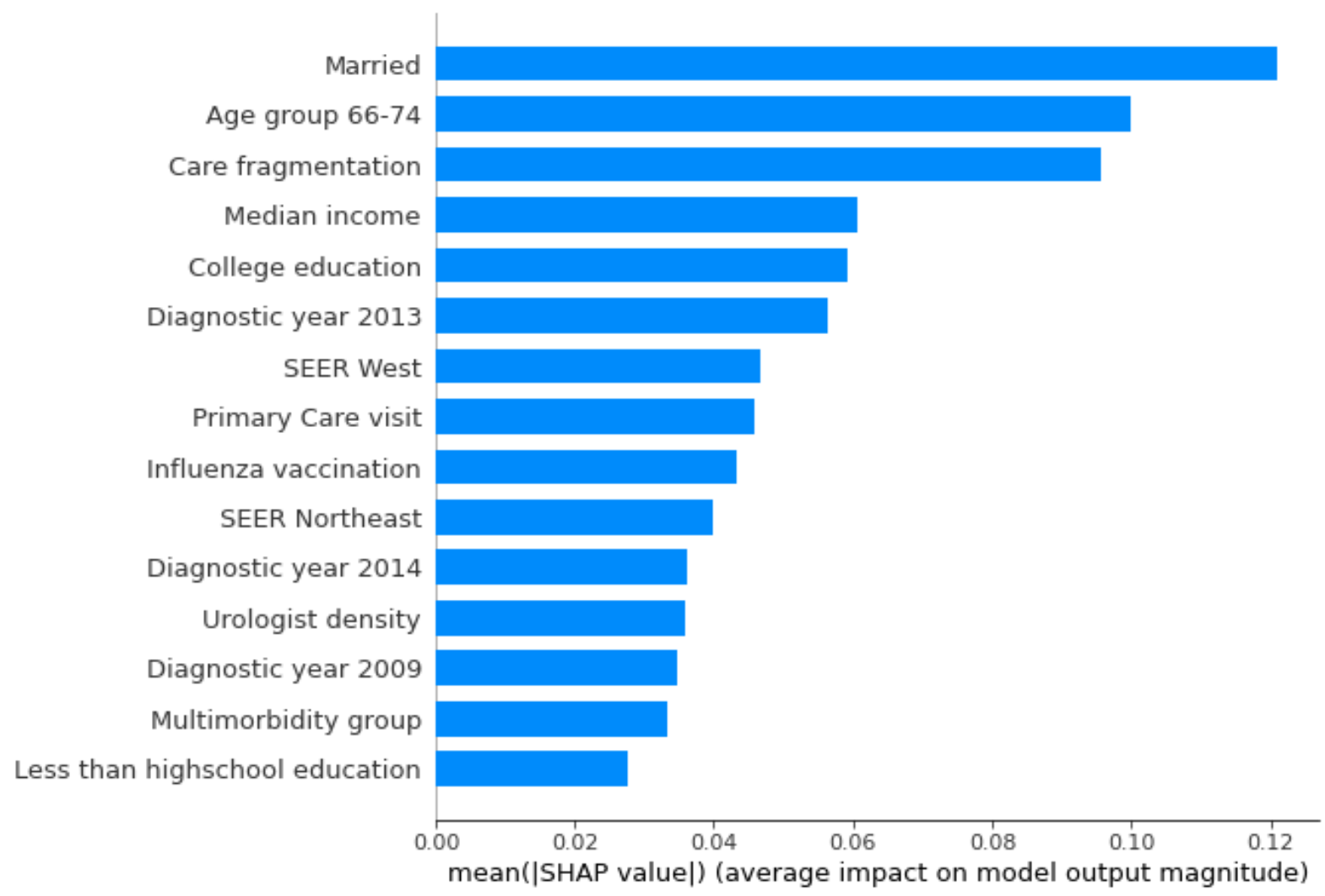

Top 15 mean SHAP values in descending order of log-odds. Based on 13870 older (age $\geq 66$ years) Fee-forService Medicare beneficiaries, with continuous enrollment in Medicare Part A \& Part B, diagnosed with incident low-risk prostate cancer (Gleason 6, PSA <10, Stage $\leq$ T2a) between 2009 and 2014. Age group included 66-74 and 75 and over categories.

Care fragmentation $=$ Bice-Boxerman continuity of care index to calculate care fragmentation during the 12 month baseline period (See Methods).

SEER = Surveillance, Epidemiology, and End Results cancer Registry. 
Figure 2.2 Positive and Negative Feature Relationships of Treatment Prediction among Fee-for-Service Medicare Beneficiaries with Incident Low-risk Prostate Cancer using SEER Cancer Registry, 2009-2014 $(n=13870)$

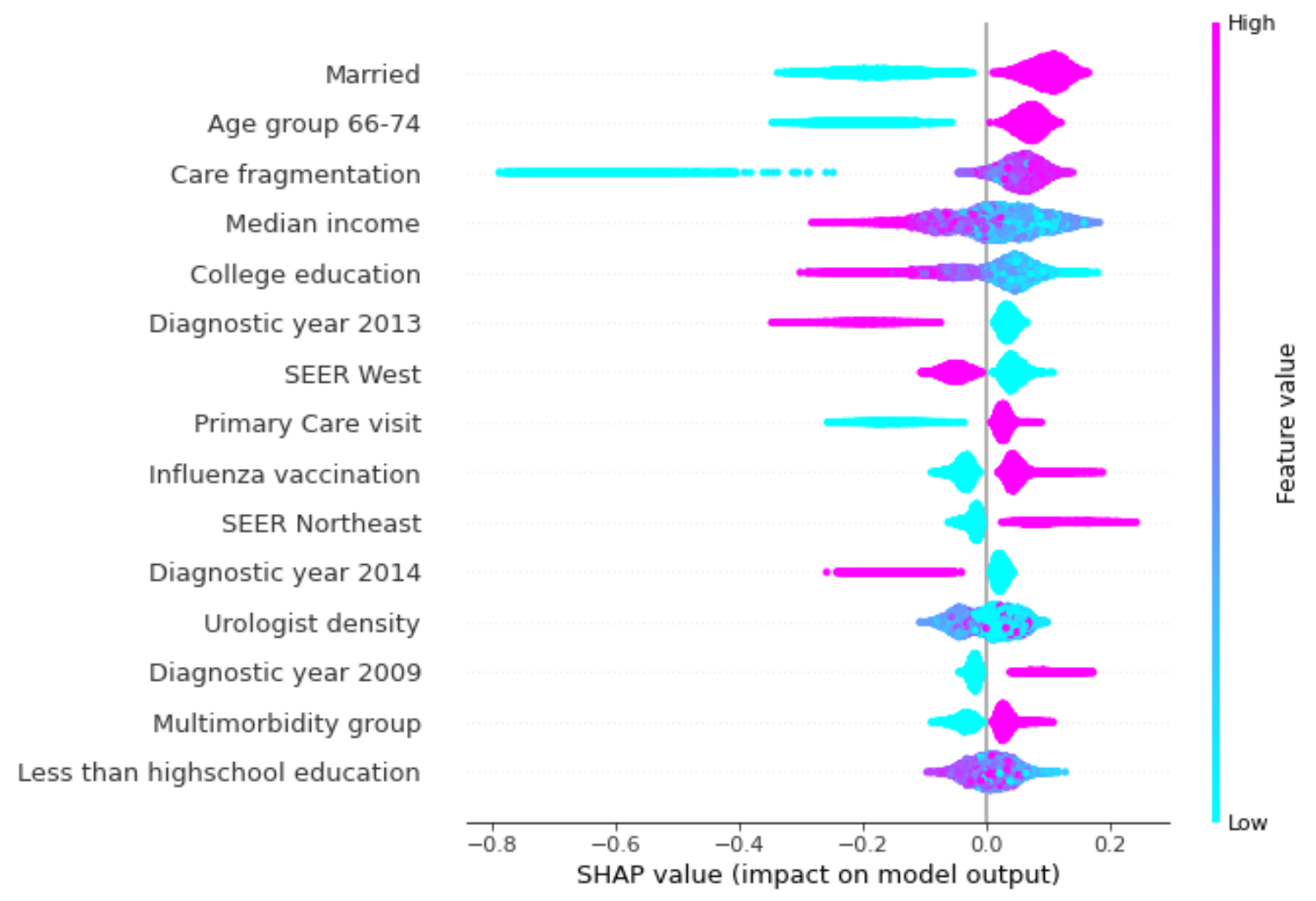

Mean SHAP values in descending order of log-odds. Based on 13870 older (age $\geq 66$ years) Fee-for-Service Medicare beneficiaries, with continuous enrollment in Medicare Part A \& Part B, diagnosed with incident lowrisk prostate cancer (Gleason 6, PSA <10, Stage $\leq$ T2a) between 2009 and 2014.

Age group 66-74 vs. adults over age 74

Multimorbidity group $=3$ or more chronic conditions.

Care fragmentation $=$ Bice-Boxerman continuity of care index to calculate care fragmentation during the 12 month baseline period (See Methods).

SEER = Surveillance, Epidemiology, and End Results cancer Registry. 
Figure 2.3 SHAP Partial Dependence Plot of Top 4 Predictive Features of Treatment among Fee-for-Service Medicare Beneficiaries with Incident Low-risk Prostate Cancer using SEER Cancer Registry, 2009-2014 $(n=13870)$

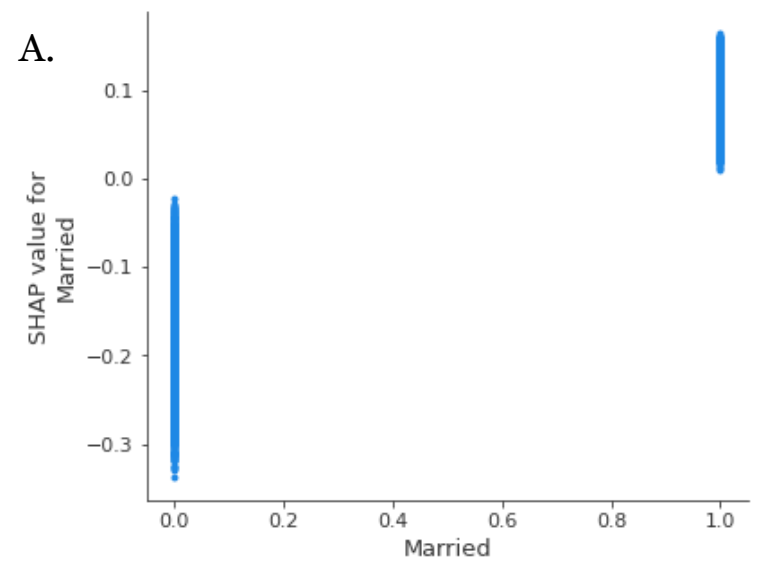

C.

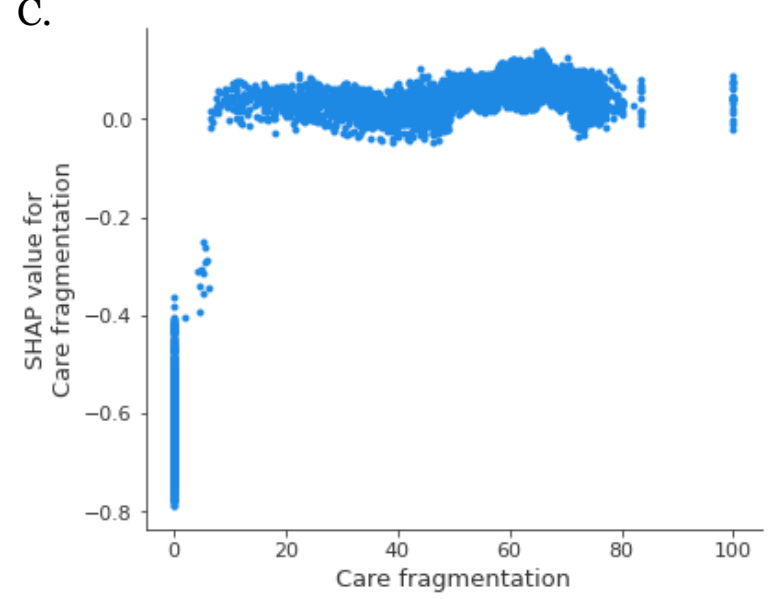

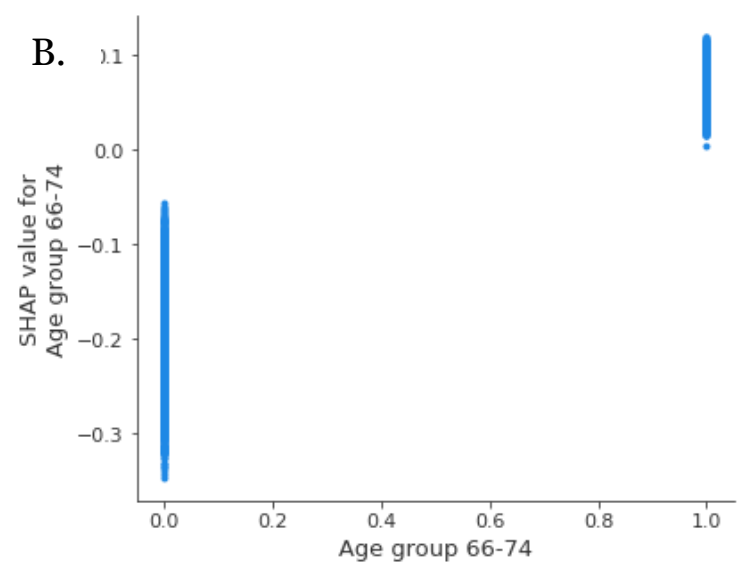

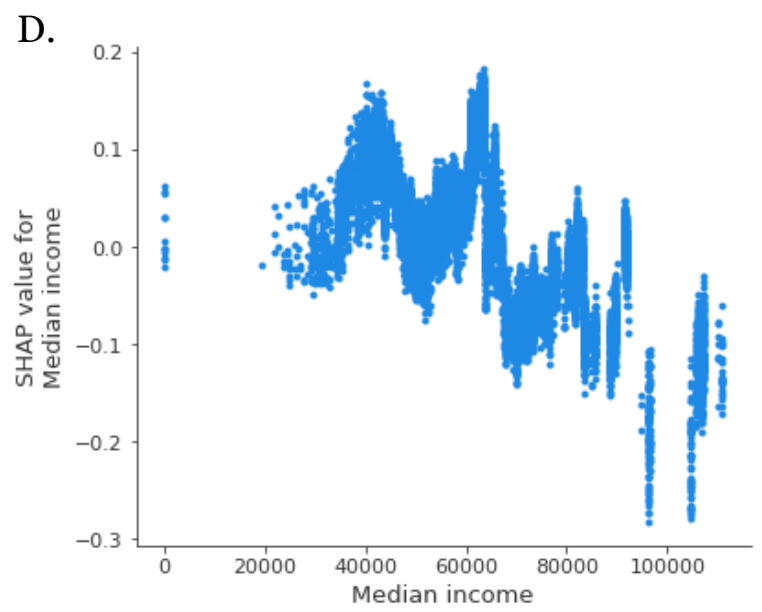

SHAP Partial Dependence plots (PDP) of log-odds SHAP values by (A.) Marital status "Married”, (B.) Age group 66-74, (C) Care fragmentation, and (D) Median Income. Based on 13870 older (age $\geq 66$ years) Fee-forService Medicare beneficiaries, with continuous enrollment in Medicare Part A \& Part B, diagnosed with incident low-risk prostate cancer (Gleason 6, PSA <10, Stage $\leq$ T2a) between 2009 and 2014.

Age group 66-74 vs. adults over age 74

Care fragmentation $=$ Bice-Boxerman continuity of care index to calculate care fragmentation during the 12 month baseline period (See Methods).

Median income $=$ County-level median income from Area Health Resource File linkage.

SHAP = Shapley Additive ePlanations.

SEER = Surveillance, Epidemiology, and End Results cancer Registry. 
Figure 2.4 SHAP Partial Density Plot of Life Expectancy and Multimorbidity Predictive Features on Treatment among Fee-for-Service Medicare Beneficiaries with Incident Low-risk Prostate Cancer using SEER Cancer Registry, 2009-2014 ( $\mathrm{n}=13870)$
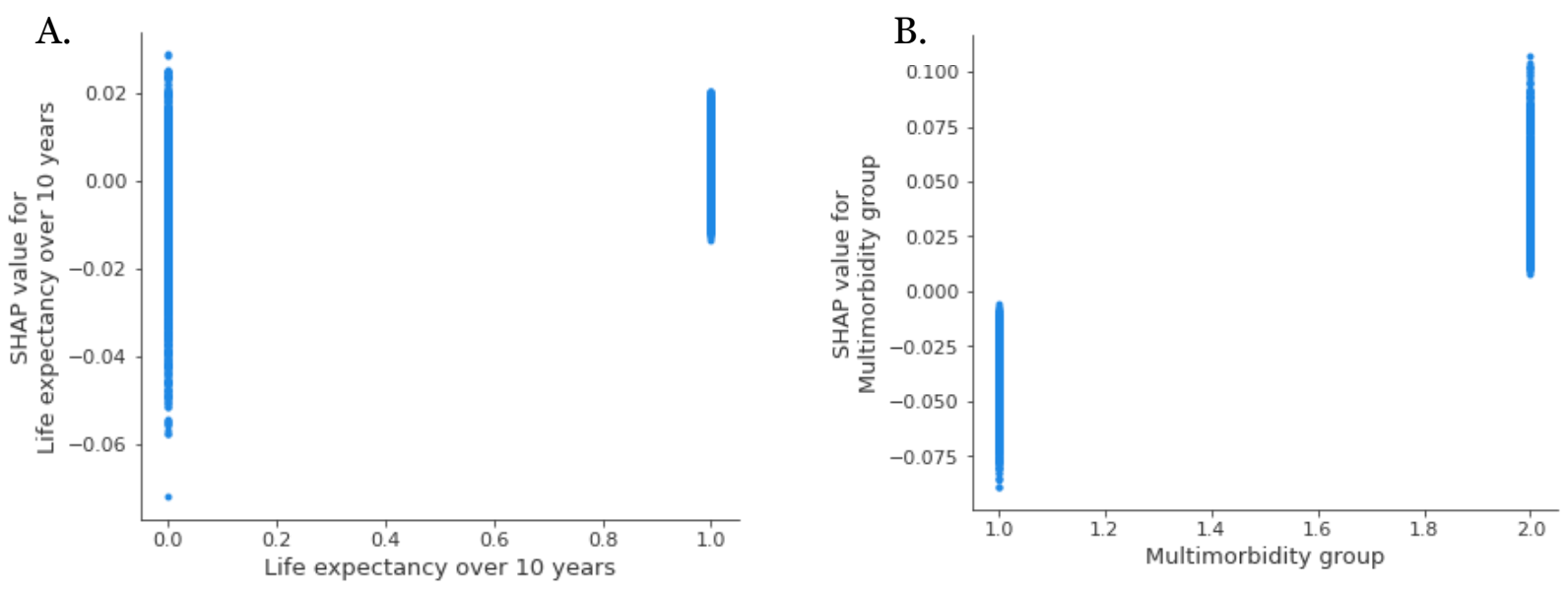

SHAP Partial Dependence plots (PDP) of log-odds SHAP values by (A.) PCCI "Life expectancy over 10 years", (B.) "Multimorbidity". Based on 13870 older (age $\geq 66$ years) Fee-for-Service Medicare beneficiaries, with continuous enrollment in Medicare Part A \& Part B, diagnosed with incident low-risk prostate cancer (Gleason 6, PSA <10, Stage $\leq$ T2a) between 2009 and 2014.

PCCI $=$ Prostate Cancer Comorbidity Index.

Multimorbidity defined as 1 = two or fewer non-cancer chronic conditions, 2 = three or more non-cancer chronic conditions.

SHAP = Shapley Additive ePlanations.

SEER = Surveillance, Epidemiology, and End Results cancer Registry. 


\section{CHAPTER 3}

\section{Associations of Multimorbidity and Patient-reported Experiences of Care with Conservative Management among Elderly Patients with Localized Prostate Cancer}

\subsection{Abstract}

Background: Many elderly localized prostate cancer patients could benefit from conservative management (CM). This retrospective cohort study examined associations of patient-reported access to care and multimorbidity on CM use patterns among Medicare Fee-for-Service (FFS) beneficiaries with localized prostate cancer.

Methods: We used linked Surveillance, Epidemiology and End Results cancer Registry, Medicare Claims, and the Medicare Consumer Assessment of Healthcare Providers and Systems survey files. We identified FFS Medicare Beneficiaries (Age $\geq 66$; continuous enrollment in Parts A \& B) with incident localized prostate cancer from 2003-2013 and a completed MCAHPS surveys within 12-months of diagnosis (n=496). We used multivariable models to examine MCAHPS measures (getting needed care, timeliness of care, and doctor communication) and multimorbidity on CM use.

Results: Localized prostate cancer patients with multimorbidity were less likely to use CM (adjusted odds ratio $(\mathrm{AOR})=0.42(0.27-0.66), \mathrm{P}<0.001)$; those with higher scores on timeliness of care $(\mathrm{AOR}=1.21(1.09$, 1.35), $\mathrm{P}<0.001)$, higher education attainment (3.21=AOR (1.50,6.89), $\mathrm{P}=0.003)$, and impaired mental health status $(4.32=\mathrm{AOR}(1.86,10.1) \mathrm{P}<0.001)$ were more likely to use $\mathrm{CM}$.

Conclusion(s): Timeliness of care was significantly and positively associated, and multimorbidity, significantly and inversely associated with CM use. Addressing specific modifiable barriers to timely care along the cancer continuum for elderly localized prostate cancer patients with limited life expectancy, and the reasons for lower CM use among those with multimorbidity, could reduce the adverse effects of overtreatment on health outcomes and costs.

Text pages: 17, Tables: 4, and 2 Figures

Keywords: prostate cancer, conservative management, active surveillance, multimorbidity, patient-centered care. 


\subsection{Introduction}

Conservative management (CM) has emerged as a common disease management approach for older adults with localized prostate cancer.(63) CM use is supported by high-level evidence for localized prostate cancer patients with low or favorable intermediate risk disease or higher risk disease with limited life expectancy.(6) CM includes protocols for low or intermediate risk prostate cancer, such as follow-up biopsies and PSA testing, or "watchful waiting" for patients with less than 5 years of life expectancy. Use of CM among patients with low-risk prostate cancer or limited life expectancy improves health-related quality of life (ie, urinary, bowel, and/or erectile dysfunction) and could reduce excessive annual health-care costs of overtreatment by $\$ 1.2$ billion. $(10,64)$

CM decisions are complex as 60\% of older adults (age > 65 years) with localized prostate cancer have pre-existing multimorbidity.(47,48) Multimorbidity affects life expectancy(47) and more than 50\% of patients with multimorbidity seek care from three or more healthcare specialists.(50) Patients with multimorbidity and cancer may be vulnerable to poor quality of cancer care and have prompted greater attention in measuring, monitoring, and incentivizing patient-centered care.(15) Measures of patient-centered care, such as patientreported experiences with care include domains of physician communication, timely care, and perceptions of getting needed care, are increasingly used as quality measures by health plans, medical groups, and physician practices. Positive patient-reported experience scores are associated with adherence to medical advice, improved clinical outcomes, and lower utilization of unnecessary health-care services(29,74) such as overtreatment of low-risk localized prostate cancer.

Patient-reported experiences may differ by multimorbidity status, which may further complicate or facilitate treatment choices for low-risk prostate cancer.(75) Identifying specific measures of patient-reported experiences that facilitate CM use among patients with incident localized prostate cancer and multimorbidity is needed to promote evidence-based cancer care.(30) For example, in colorectal cancer populations, patientreported experiences with perceived timely care are associated with evidenced-based followup.(31) Understanding the relationship between patient-reported experiences of care on CM use can inform patient-centered care approaches to improve adoption of CM use, thereby reducing the adverse effects of overtreatment among older patients with multimorbidity and localized prostate cancer. 
Despite the importance of patient-reported experiences, CM studies primarily focus on disease characteristics, clinical, and sociodemographic factors. $(27,38,39,63)$ To date, no studies have investigated the impact of patient-reported experiences on CM use among medically complex patients with localized prostate cancer. Therefore, the primary objective of this study is to examine the associations of multimorbidity and patient-reported experiences on CM use among Fee-for-Service (FFS) Medicare beneficiaries with localized prostate cancer using Consumer Assessment of Healthcare Providers and Systems (MCAHPS $®$ ) patient surveys and Medicare claims linkages.

\subsection{Methods}

The study cohort included men diagnosed with localized prostate cancer defined as American Joint Committee on Cancer stage T2a or less,(6) aged 66 or older, with continuous enrollment in FFS Medicare Parts A and B throughout the study period (Figure 3.1).

Date of incident localized prostate cancer diagnosis was used as an index date and 12 months before diagnosis was used as the baseline period. During the baseline period, we identified multimorbidity using Medicare claims and calculated life expectancy estimates.

We also defined the "CM measurement" period as 12 months after diagnosis. During this period, we identified CM based on validated methods for claims data.(53)

As MCAHPS surveys can be administered at varying points during the post diagnosis period, we followed individuals for an additional period of 12 months. Thus, our follow-up period consisted of 24 months after incident localized prostate cancer diagnosis.

To account for varying months from diagnosis to survey administration, we included time from diagnosis to survey as one of the independent variables in the models. However, as this variable was not significant and did not affect our main results, we did not include time from diagnosis to survey administration variable in the final model. As a sensitivity analysis, we also estimated CM use during 24 months after diagnosis (Appendix 7.3).

\section{Data sources}

The Surveillance, Epidemiology and End Results (SEER) cancer registry contains tumor and demographic information for patients diagnosed with cancer while residing in a SEER region. We derived 
Medicare eligibility from the SEER data (Figure 3.1). We extracted fee-for-service Medicare claims from Medicare Provider Analysis and Review (MEDPAR), Carrier Claims, Outpatient Claims, Home Health Agency, and Durable Medical Equipment files.

Medicare Consumer Assessment of Healthcare Providers \& Systems (MCAHPS $®$ ) surveys, administered by the CMS, use standardized and validated questionnaires to collect information on patientreported experiences with health-care providers.(40) MCAHPS collection methodologies use a weighted probability sampling procedure covering all the 50 US states, DC, and Puerto Rico, which are then linked to SEER patients. $(40,41)$

Area Health Resource File (AHRF) files were linked via MEDPAR FIPS state and county codes and were used to calculate radiation oncologist and urologist densities.(42) Census files were linked to calculate countylevel median income quartiles.

\section{Dependent Variable}

We operationalized CM use as the absence of curative treatment within 12 months after incident localized prostate cancer. Treatment was identified using International Classification of Diagnosis 9th edition (ICD9), ICD9 procedure codes, and Healthcare Common Procedure Coding System (HCPCS) codes from FFS Medicare claims (Appendix 7.4).(6,38,53)

\section{Key Independent Variables}

The multimorbidity framework developed by the United States Department of Health and Human Services for guiding programs, practice, and policy guided the selection of chronic conditions as follows: arthritis, asthma, coronary artery disease, congestive heart failure, chronic kidney disease, chronic obstructive pulmonary disease, cardiac arrhythmias, acute myocardial infarction, dementia, diabetes, depression, hepatitis, hyperlipidemia, hypertension, human immunodeficiency virus, osteoporosis, substance abuse, schizophrenia, stroke, anemia, and lower limb fracture.(55) The most common definition of multimorbidity is the concurrent presence of two or more conditions in the same individual.(76) We defined multimorbidity as the presence of three or more conditions in the same individuals as older men diagnosed with prostate cancer at age 65 or higher are at high risk for competing risk mortality. For example, among men with three or more 
comorbid conditions, aged 61-74 and 75 years or older, 10-year other cause mortality is 40\% and 71\%, respectively.(77)

Prostate cancer comorbidity index (PCCI), a weighted comorbidity index validated prostate cancer patient populations, was used to predict 5- and 10-year life expectancy in prostate cancer patient populations.(77) PCCI was calculated during the baseline period to estimate 5- and 10-year life expectancy. PCCI was categorized into three groups: 0-8 (>10-year life expectancy); 9 to 13 (5- to 10-year life expectance); and $>13$ (<5-year life expectancy). In all models, PCCI total o-8 was used as the reference group.

Published research in prostate cancer patients often uses Charlson Comorbidity Index (CCI); therefore, we conducted a supplemental analysis using CCI. In these analyses, CCI scores were dichotomized with “o-1" as the reference group.(78)

We included three MCAHPS composite measures - "getting needed care," "getting care quickly," and "doctor communication"-which rates the ability to get needed appointments, timeliness of care when care is needed, and how well the physician communicated. Patients report experiences with health-care access in the last 6 months. MCAHPS surveys have been extensively validated for measuring patient-reported access to care and are commonly used for quality improvement as well as value-based purchasing initiatives.(40) MCAHPS are based on a $0-100$ scale with o representing the lowest and 100 representing the highest score; we examined the effect of 10 unit changes in composite items on the dependent variable.

Management of preexisting multimorbidity and shared prostate cancer treatment decision-making requires the use of limited resources (ie, time to manage chronic conditions and availability of health-care professionals and resources). Therefore, for other independent variables, the competing demands model was used to conceptualize factors known to affect localized prostate cancer treatment selection within clinician, patient, and practice ecosystem domains.(38,53,79) (Figure 3.2).

Multivariable models were adjusted with independent variables: diagnosis year group (2003-2009 and 2010-2013), low-risk prostate cancer (operationalized as Gleason Score $\leq 6$ and PSA test $\leq 10 \mathrm{ng} / \mathrm{mL}$ or Gleason Score $>=7$ or PSA $>=10 \mathrm{ng} / \mathrm{mL}$ ), self-reported general and mental health status, education-level, zipcode income quartiles, and county-level quartiles of urologists and radiation oncologists per 10,000 persons over age 65 . 
Our analyses include several case-mix adjustment variables such as age, education, general health status, mental health status, income level, and race. Secondary analyses using additional recommended casemix adjustment variables, such as dual-eligible Medicaid respondents, "proxy" survey completion, and time from cancer diagnosis to survey completion, did not significantly improve model specification.(80)

To assess the potential influence of missing data, we examined missing data patterns using covariatedependent missingness methods.(81) Mean values were imputed to independent variables of interest. For categorical variables, including general and mental health status, missing data indicators were created and included as a separate category in the regression models.

Chi-square tests and t tests were used to identify significant group differences in CM use by categorical variables. Multivariable models were fit using separate unadjusted and adjusted logistic regressions to identify independent and interactive associations of multimorbidity and patient care experiences on CM use. All statistical tests were two-sided with a 5\% Type I error rate and were completed in STATA (StataCorp, College Station, TX).

\subsection{Results}

The study cohort was predominantly non-Hispanic, whites (84.5\%). The median age at diagnosis was 72.8 years and did not differ by year of diagnosis $(2003-2009: 73.6=\mathrm{M}, 5.38 ; 2010-2013: 73.6=\mathrm{M}, \mathrm{SD}=5.14)$. Average composite scores for doctor communication, getting needed care, and getting care quickly were 91.0 $(\mathrm{SD}=12.2), 88.6(\mathrm{SD}=15.6)$, and $70.8(\mathrm{SD}=21.7)$, respectively.

Overall, 33.5\% used CM, defined as no curative treatment within 12 months of incident localized prostate cancer diagnosis. Use of CM was only marginally higher in men with low-risk relative to those with higher-risk disease ( $\leq$ cT2a and PSA $>=10 \mathrm{ng} / \mathrm{mL}$ or Gleason Score $>6)(38.7 \%$ vs 30.9\%, respectively, $\mathrm{P}=.08)$ (Table 3.1). High-school graduation, college education, low-risk prostate cancer diagnosis, and mental health status were significantly more frequent among patients using CM (Table 3.1). CM use by localized prostate cancer patients with higher-risk disease was 30.9\%. Higher-risk disease was significantly more common among age groups $75+(75.9 \%)$ vs $66-74(62.4 \%)(\mathrm{P}=.002)$ and significant differences by patients with multimorbidity 24.6\% $(n=45)$ vs those without multimorbidity $38.7 \%(n=58),(X 2=7.65, P=.006)$ were observed. 
In our study cohort, $57.2 \%$ had multimorbidity. Patients 75 years or older were significantly more likely to have multimorbidity than those aged 66-74 years (64.4\% vs 53.4\%). Blacks had a higher percentage of multimorbidity as compared to whites (76.1\% vs 53.9\%). Patients with multimorbidity using treatment $(\mathrm{n}=$ 207) did not differ significantly by patient, clinician, or practice ecosystem factors except for mental health status of excellent/very good (74.6\%) and good (77.5\%) vs patients using CM (P = .031). Patients with multimorbidity and higher-risk disease $(n=183)$ significantly more frequently used treatment if aged 66-74 $(82.5 \%)(\mathrm{P}=.011)$. Average composite scores for doctor communication, getting needed care, and getting care quickly did not differ by multimorbidity status. CM use was significantly lower in men with vs without multimorbidity (27.1\% vs $72.9 \%$, respectively, $\mathrm{P}<.001)$.

Average getting care quickly composite scores (ie, timely care) were higher for those with CM use as compared to those without CM use (Table 3.2). CM use significantly differed by PCCI categories, with lower percentages among groups with less than 10 (27.0\%) and 5 (27.0\%) vs more than 10 (38.9\%) years of life expectancy $(\mathrm{X} 2=7.82, \mathrm{P}=.020)($ Table 3.2$)$.

PCCI life expectancy groups did not statistically differ by CM use. Higher-risk patients reporting fair or poor mental health status $(62.1 \% ; \mathrm{P}=.002)$ vs excellent mental health status, and college education $(33 \%)$ or high-school graduates (37.5\%) vs no high-school graduation (13.7\%), significantly used CM more frequently. Getting care quickly composite scores were significantly higher among higher-risk patients ( $\mathrm{n}=333) \mathrm{using} \mathrm{CM}$ $(\mathrm{M}=75.8)$ vs curative treatment $(\mathrm{M}=69.8),(\mathrm{t}=-2.43, \mathrm{CI} 95 \%=69.3,73.9, \mathrm{P}=.016)$.

Multimorbidity was significantly and inversely related to CM use in unadjusted logistic regression analyses (odds ratios $(\mathrm{OR})=0.55 ; 95 \% \mathrm{CI}=0.35,0.75)$. Adjustment for other factors, including timeliness of care, further strengthened this association (adjusted $\mathrm{OR}(\mathrm{AOR})=0.42$, CI 0.27- 0.66) (Table 3.3); additional models adjusting for other patient experience domains or CCI were not significant. Getting care quickly showed a significant, positive association with $\mathrm{CM}$ use in both the unadjusted analyses $(\mathrm{OR}=1.15 ; 95 \% \mathrm{CI}=1.05,1.27)$ and the fully adjusted models $(\mathrm{AOR}=1.21 ; 95 \% \mathrm{CI}=1.09,1.34)$. In models including PCCI life expectancy categories, less than 10- and 5-year life expectancy were inversely associated with CM use (Appendix 7.2).

CM use was also significantly and positively associated with fair/poor mental health status, low-risk prostate cancer diagnosis, college education or more, and high-school graduation in all adjusted models (Table 
3.3; Appendix 7.1 and 7.2). We found no evidence for a modifying effect of patient-experience variables, multimorbidity, PCCI, or other independent variables on the observed associations.

\subsection{Discussion}

In this study, we assessed the independent associations of multimorbidity and patient-reported experiences with care on CM use among older men with localized prostate cancer. Despite proven benefits of $\mathrm{CM}$, one in three (33.5\%) of all men with localized prostate cancer, and only two in five (41\%) men over the age of 75 years, used CM. Our estimates of CM use among patients with localized prostate cancer are lower than those reported in recent investigations using SEER-Medicare data (42.1\% in 2015)(63) but higher than reported in an investigation of Michigan Medicare beneficiaries (22.3\% in 2014).(53) We speculate that these differences could be due to variation in study population characteristics (ours included prostate cancer patients from many regions of the US) and geographic practice patterns.(82-84)

Multimorbidity and life expectancy are critical components of patient counseling after a localized prostate cancer diagnosis as many older men do not live long enough to benefit from treatment. Patients with low or favorable intermediate-risk disease or higher-risk disease with limited life expectancy could avoid significant urinary, erectile, and rectal treatment morbidities without increasing the risk of prostate cancerspecific mortality with CM. $(85,86)$ However, in our study, men with multimorbidity were significantly less likely to use CM compared to those without multimorbidity after controlling for age, low-risk prostate cancer, and other sociodemographic variables. We speculate that men with multimorbidity and low-risk cancer may not opt for treatment because they may have a preference for immediate cure (ie, "take care of it")(87) and may not want to add one more condition that requires long-term management. Furthermore, men with

multimorbidity may fear nontreatment regret,(88) emotional distress,(89) and anxiety.(90) Strong multidisciplinary management strategies, including significant psychological support from primary care physicians and specialists (ie, urologist and/or medical and radiation oncologists), are needed to mitigate decisional conflict(91) and therefore facilitate CM use.(27)

In adjusted models, including validated life expectancy measures for prostate cancer survivors, patients meeting evidence-based criteria2 for CM were $58 \%$ less likely to use CM based on life expectancy alone (ie, less than 5 years). Previous studies using CCI report both positive and negative relationships between comorbidity 
burden and CM use in Medicare FFS populations. $(67,68,92)$ In a supplemental analysis in this study, CCI was not significantly associated with CM use. Taken together, these findings suggest that clinical and population differences in comorbidity definitions are likely to account for mixed findings in several previous investigations.(69) By defining multimorbidity using a list of conditions prioritized by policy makers in the US,(55) our study made a unique contribution to this field. However, as pointed out by a systematic review that current life expectancy prediction tools lack both practical and theoretical utility,(34) comorbidity measures that can be easily operationalized in a clinical setting are needed. Recently, age-adjusted indexes, such as the PCCI used in our study, were developed to provide life expectancy estimates in patients with prostate cancer.(54) Certain types (cardiovascular disease) and combinations (cardiometabolic and respiratory)(65) of chronic conditions are associated with treatment regardless of patient, clinician, and health-care ecosystem factors. Additional research is needed to understand the relationship between more precise estimates of life expectancy and multimorbidity on CM use in FFS Medicare populations.

In our study, patient-reported experiences, specifically timeliness of care, were positively associated with CM use. Patients with higher timeliness of care scores were significantly more likely to use CM after adjusting for demographic, clinical, socioeconomic, and health-care system factors. Timely access to care for localized prostate cancer patients is not limited to initial diagnosis of prostate cancer, but the opportunity and ease by which a patient is able to utilize needed services along the continuum of care throughout survivorship.(93) Choices for elderly localized prostate cancer patients involve selecting curative and noncurative treatments with trade-offs in efficacy, potential adverse quality of life effects, and competing risk mortality. MCAHPS timeliness of care domains, such as perceived barriers to appointment scheduling, are fundamental to shared decision-making among multiple health-care providers that significantly influence localized prostate cancer treatment choice.(94-96) We speculate that patients with higher timely care ratings may choose CM because they may have a favorable perception of health-care system capacity to provide services once a need is detected.

Our study findings have important policy implications. Currently, no value-based mechanisms exist to support the use of CM in Medicare FFS populations. Existing literature also suggest that CM use in FFS system varies among physician practices by 5.1\%-71.2\%.(84) Emerging oncology care models by CMS may need to 
incorporate risk-adjusted $\mathrm{CM}$ use as a quality indicator along the cancer care continuum(97) to promote $\mathrm{CM}$ use among men with localized prostate cancer. Recently, the NCCN Quality and Outcomes committee identified significant gaps in evaluating high-quality cancer care with patient experience measures and evidence-based practice.(85) More research is needed to identify specific barriers to timely care and how validated patient-reported experience measures can be used to support evidence-based management of localized prostate cancer patients in oncology care models.

We also observed that elements of social determinants, such as education, were associated with CM use. Although educational attainment may not be modifiable among older adults, initiatives such as "health in all policies” by World Health Organization and the Centers for Disease and Prevention Control “integrate and articulated health considerations" into community health policy.(73) These experts concluded that social, economic, and physical environments have a significant impact on the health of an individual and these effects should be considered in the development of all public policies and programs.

\subsection{Strengths and Limitations}

The SEER-CAHPS data linkage is a robust and unique resource that provides an ideal opportunity to study patient-centered care delivery of contemporary treatment patterns among patients with localized prostate cancer and multimorbidity. We build on previous findings by including validated estimations of life expectancy and definitions of multimorbidity to access the impact of comorbid conditions on patterns of contemporary treatment options for older localized prostate cancer patients.

Our study results must be interpreted with important limitations. MCAHPS surveys request patientreported experiences with care "in the last 6 months".(40) Due to relatively small sample size, we included surveys completed within 6 months after localized prostate cancer diagnosis which may overlap with the baseline period. However, our results were robust to multivariable adjustments for time between cancer diagnosis and survey completion. Due to MCAHPS survey administration processes and collection, we cannot directly attribute MCAHPS composite ratings to physician specialty or the prostate cancer diagnosis; instead, our results are generalizable to the entire patient experience after diagnosis which may include multiple care providers for multiple conditions. The study sample comprised of predominantly non-Hispanic white, urban 
adults, potentially limiting generalizability to ethnic minorities, rural, or other populations. Our study was restricted to Medicare FFS enrollees 65 years or older and may not be generalizable to younger adults or individuals on private insurance. Lastly, due to sample size limitations, we were unable to analyze the relationship of individual chronic conditions with CM use.

\subsection{Conclusions}

Our results highlight the effect of patient-reported experiences, multimorbidity, and life expectancy on CM use among older men with localized prostate cancer. While factors such as multimorbidity and life expectancy are critical clinical components that may affect the choice of CM vs over treatment, our study highlights the role of nonclinical factors, specifically patient-reported experiences with care on treatment of localized prostate cancer. Our findings support a "population health-based" oncology care model in which both clinical and nonclinical factors, such as patient-reported experiences, are integrated to promote CM use and avoid overtreatment among older men with localized prostate cancer. 
Table 3.1 Patient Characteristics by Conservative Management among Fee-for-Service Medicare Beneficiaries with Incident Localized Prostate Cancer using Linked SEER Cancer Registry with MCAHPS, 2002-2013 $(n=496)$

\begin{tabular}{|c|c|c|c|c|c|c|c|}
\hline & & & & No & & & \\
\hline & & $\mathbf{N}$ & $\%$ & $\mathbf{N}$ & $\%$ & $\mathbf{X}^{2}$ & p-value \\
\hline ALL & & 166 & $33 \cdot 5$ & 330 & 66.5 & & \\
\hline Age il & ars & & & & & 1.32 & 0.250 \\
\hline & $66-74$ & 102 & 31.7 & 220 & 68.3 & & \\
\hline & $75^{+}$ & 64 & 36.8 & 110 & 63.2 & & \\
\hline Race & & & & & & 0.07 & 0.964 \\
\hline & White & 140 & 33.4 & 279 & 66.6 & & \\
\hline & Black & 15 & 32.6 & 31 & 67.4 & & \\
\hline & Other & 11 & $35 \cdot 5$ & 20 & 64.5 & & \\
\hline Marit & tatus & & & & & 0.2 & 0.905 \\
\hline & Married & 115 & 32.9 & 235 & 67.1 & & \\
\hline & Unmarried & 22 & 34.9 & 41 & 65.1 & & \\
\hline & Unknown & 29 & 34.9 & 54 & 65.1 & & \\
\hline Incon & [uartiles & & & & & 0.54 & 0.909 \\
\hline & First & 38 & $33 \cdot 3$ & 76 & 66.7 & & \\
\hline & Second & 39 & 33.6 & 77 & 66.4 & & \\
\hline & Third & 38 & 31.1 & 84 & 68.9 & & \\
\hline & Four & 51 & 35.4 & 93 & 64.6 & & \\
\hline Educ: & & & & & & 8.11 & $\mathbf{0 . 0 1 7}$ \\
\hline & College or more & 100 & 36.6 & 173 & 63.4 & & \\
\hline & High School Grad. & 42 & 35.9 & 75 & 64.1 & & \\
\hline & No High School Grad. & 13 & 18.8 & 56 & 81.2 & & \\
\hline Gene & nealth status & & & & & $3 \cdot 32$ & 0.19 \\
\hline & Excellent/Very Good & 59 & $35 \cdot 5$ & 107 & 64.5 & & \\
\hline & Good & 54 & 28.6 & 135 & 71.4 & & \\
\hline & Fair/Poor & 47 & 37.6 & 78 & 62.4 & & \\
\hline Ment & ealth status & & & & & 11.3 & 0.004 \\
\hline & Excellent/Very Good & 104 & 31.6 & 225 & 68.4 & & \\
\hline & Good & 34 & 30.1 & 79 & 69.9 & & \\
\hline & Fair/Poor & 22 & 57.9 & 16 & 42.1 & & \\
\hline
\end{tabular}

Bold values denote statistical significance at the $P$-value $<.05$ level.

Based on 496 older (age $\geq 66$ years) Fee-for-Service Medicare beneficiaries, with continuous enrollment in Medicare Part A \& Part B, diagnosed with incident localized prostate cancer between 2003 and 2013. Abbreviations: CM, Conservative management; MCAHPS, Medicare Claims and the Medicare Consumer Assessment of Healthcare Providers and System surveys; SEER, Surveillance, Epidemiology, and End Results cancer Registry. 


\begin{tabular}{|c|c|c|c|c|c|c|}
\hline \multicolumn{7}{|l|}{ Table 3.1 "continued" } \\
\hline Urologist density & & & & & 4.99 & 0.173 \\
\hline o to 1.41 & 41 & 33.1 & 83 & 66.9 & & \\
\hline 1.41 to 2.49 & 33 & 26.6 & 91 & 73.4 & & \\
\hline 2.5 to 3.46 & 51 & 39.8 & 77 & 60.2 & & \\
\hline 3.47 to 10.2 & 41 & 34.2 & 79 & 65.8 & & \\
\hline Radiation oncologist density & & & & & 5.43 & 0.143 \\
\hline o to 0.44 & 37 & 29.8 & 87 & 70.2 & & \\
\hline 0.45 to 1.07 & 41 & 33.1 & 83 & 66.9 & & \\
\hline 1.07 to 1.49 & 52 & 41.6 & 73 & 58.4 & & \\
\hline 1.51 to 5.35 & 36 & 29.3 & 87 & 70.7 & & \\
\hline SEER region & & & & & 5.09 & 0.166 \\
\hline Northeast & 33 & 35.5 & 60 & 64.5 & & \\
\hline South & 35 & 30.4 & 80 & 69.6 & & \\
\hline North-central & 13 & 22.4 & 45 & 77.6 & & \\
\hline West & 85 & 37 & 145 & 63.0 & & \\
\hline Metro Status & & & & & 0.71 & 0.401 \\
\hline Metro & 138 & 34.3 & 264 & 65.7 & & \\
\hline Non-Metro & 28 & 29.8 & 66 & 70.2 & & \\
\hline Diagnosis Year & & & & & 0.44 & 0.509 \\
\hline 2002-2007 & 96 & 32.3 & 201 & 67.7 & & \\
\hline 2008-2013 & 70 & 35.2 & 129 & 64.8 & & \\
\hline Low-risk prostate cancer & & & & & 2.93 & 0.087 \\
\hline Yes & 63 & 38.7 & 100 & 61.3 & & \\
\hline No & 103 & 30.9 & 230 & 69.1 & & \\
\hline
\end{tabular}

Bold values denote statistical significance at the $P$-value $<.05$ level.

Based on 496 older (age $\geq 66$ years) Fee-for-Service Medicare beneficiaries, with continuous enrollment in Medicare Part A \& Part B, diagnosed with incident localized prostate cancer between 2003 and 2013. Abbreviations: CM, Conservative management; MCAHPS, Medicare Claims and the Medicare Consumer Assessment of Healthcare Providers and System surveys; SEER, Surveillance, Epidemiology, and End Results cancer Registry. 
Table 3.2 Multimorbidity and Patient Experiences by Conservative Management among Fee-for-Service Medicare Beneficiaries with Incident Localized Prostate Cancer using Linked SEER Cancer Registry with MCAHPS, 2002-2013

\begin{tabular}{|l|c|c|c|c|c|c|}
\hline & \multicolumn{2}{|c|}{ CM } & \multicolumn{2}{c|}{ No CM } & & \\
\hline & $\mathrm{N}$ & $\%$ & $\mathrm{~N}$ & $\%$ & $\mathrm{X}^{2}$ & $\mathrm{p}$-value \\
\hline Multimorbidity & $\mathbf{1 6 6}$ & $\mathbf{3 3 . 5}$ & $\mathbf{3 3 0}$ & $\mathbf{6 6 . 5}$ & & \\
\hline Yes & & & & & 12.1 & $<0.001$ \\
\hline No & 77 & 27.1 & 207 & 72.9 & & \\
\hline PCCI & 89 & 42.0 & 123 & 58.0 & & \\
\hline$\quad<$ 5 years life expectancy & & & & & 7.82 & 0.020 \\
\hline$\geq$ 5 and < 10 years life expectancy & 20 & 27.0 & 54 & 73.0 & & \\
\hline$\geq 10$ years life expectancy & 41 & 27.0 & 111 & 73.0 & & \\
\hline Getting Needed Care & 105 & 38.9 & 165 & 61.1 & & \\
\hline Getting Care Quickly & $\mathbf{M e a n}$ & $\mathbf{S E}$ & $\mathbf{M e a n}$ & $\mathbf{S E}$ & $\mathbf{t}$ t-Value & p-value \\
\hline Doctor/Patient Communication & 87.1 & 1.33 & 89.3 & 0.81 & 1.48 & N.S. \\
\hline
\end{tabular}


Table 3.3 Unadjusted (UOR), Adjusted Odds Ratios (AOR) and 95\% Confidence Intervals (CI) of Multimorbidity, Mental Health, Timeliness of Care, Low-risk Prostate Cancer, and Education on Likelihood of Conservative Management Use among Fee-for-Service Medicare Beneficiaries with Incident Localized Prostate Cancer using Linked SEER Cancer Registry with MCAHPS, 2002-2013 ( $\mathrm{N}=496)$.

\begin{tabular}{|c|c|c|c|c|c|c|}
\hline & \multicolumn{3}{|c|}{ UOR [95\% CI] } & \multicolumn{3}{|c|}{ AOR [95\% CI] } \\
\hline \multicolumn{7}{|c|}{ Patient Experience: Getting Care Quickly } \\
\hline Multimorbidity & & & p-value & & & p-value \\
\hline Yes & 0.51 & {$[0.35-0.75]$} & 0.001 & 0.42 & {$[0.27-0.66]$} & $<0.001$ \\
\hline \multicolumn{7}{|l|}{ No (Ref.) } \\
\hline Getting Care Quickly & 1.15 & {$[1.05-1.27]$} & 0.003 & 1.21 & {$[1.09-1.35]$} & $<0.001$ \\
\hline \multicolumn{7}{|l|}{ Low-risk prostate cancer } \\
\hline Yes & 1.41 & {$[0.95-2.08]$} & 0.088 & 1.76 & {$[1.14-2.72]$} & 0.01 \\
\hline \multicolumn{7}{|l|}{ No (Ref.) } \\
\hline \multicolumn{7}{|l|}{ Mental Health Status } \\
\hline Fair/Poor & 2.97 & {$[1.50-5.90]$} & 0.002 & 4.32 & {$[1.86-10.1]$} & $<0.001$ \\
\hline \multicolumn{7}{|l|}{ Ex/VG/Good (Ref.) } \\
\hline \multicolumn{7}{|l|}{ Education } \\
\hline College or more & 2.49 & {$[1.30-4.78]$} & 0.006 & 3.21 & {$[1.50-6.89]$} & 0.003 \\
\hline High-school graduate & 2.41 & {$[1.18-4.92]$} & 0.015 & 3.53 & {$[1.59-7.83]$} & 0.002 \\
\hline \multicolumn{7}{|l|}{ No high-school graduation (Ref.) } \\
\hline \multicolumn{7}{|c|}{ Patient Experience: Getting Need Care } \\
\hline \multicolumn{7}{|l|}{ Multimorbidity } \\
\hline Yes & & & 0.088 & 0.45 & {$[0.30-0.70]$} & $<0.001$ \\
\hline \multicolumn{7}{|l|}{ No (Ref.) } \\
\hline Getting Needed Care & - & - & N.S. & - & - & N.S. \\
\hline \multicolumn{7}{|c|}{ Patient Experience: Doctor Communication } \\
\hline \multicolumn{7}{|l|}{ Multimorbidity } \\
\hline Yes & & & 0.088 & 0.45 & {$[0.29-0.68]$} & $<0.001$ \\
\hline No (Ref.) & & & & & & \\
\hline Doctor Communication & - & - & N.S. & - & - & N.S. \\
\hline
\end{tabular}


Figure 3.1 Cohort Selection and Exclusion

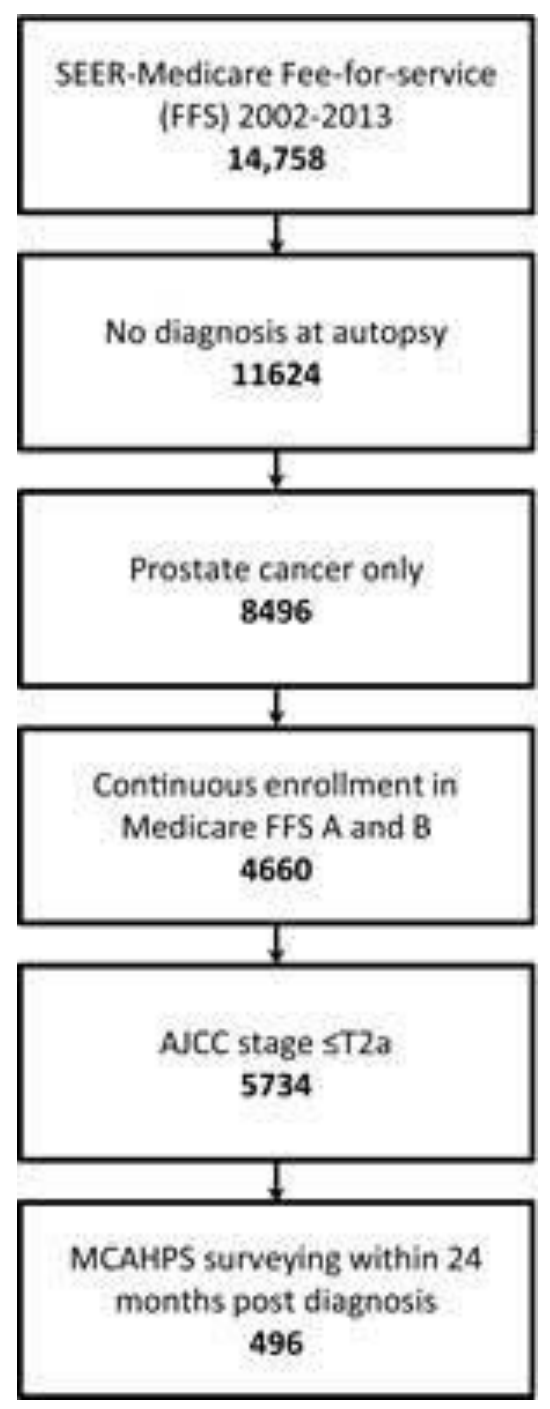


Figure 3.2 Adapted Competing Demands Framework

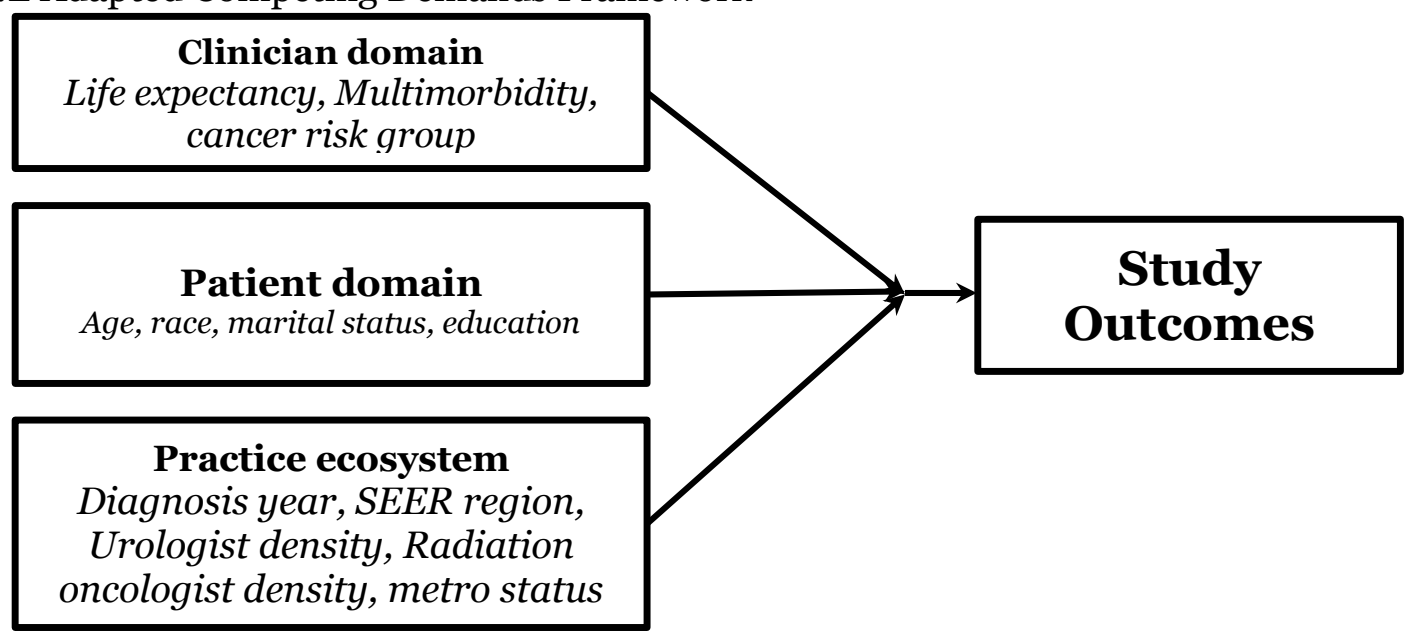




\section{CHAPTER 4}

\section{Healthcare Expenditures Associated with Low-value Care among Older Men with Incident}

\section{Localized Prostate Cancer: Statistical and Machine Learning Approaches}

\subsection{Abstract}

Background: Older adults with incident localized prostate cancer represent a large, medically complex population at risk of low-value care in the form of overtreatment. Conservative management (CM), or nontreatment of prostate cancer with little cancer specific mortality risk, can reduce the adverse effects of overtreatment. Other types of low-value care services are associated with high, compounding “downstream” costs and may be useful for predicting cancer survivorship costs.

Research Objective: Use statistical and machine learning approaches to estimate the association of lowvalue care use prior to incident prostate cancer diagnosis on average non cancer related total healthcare expenditures 12-24 months after diagnosis. Generalized linear models (GLM) with log-link and gamma distribution were used to estimate total healthcare cost. Predictive feature importances of healthcare costs and non-linear relationships were estimated using the XGBoost Machine Learning (ML) algorithm and SHapley Additive exPlanations (SHAP).

Study Design: Retrospective cohort study.

Population Studied: We used linked Surveillance, Epidemiology, and End Results cancer Registry, Medicare Claims, Census, and Area Health Resource files identify Medicare Fee-for-Service beneficiaries with continuous enrollment in Parts A \& B and incident localized prostate cancer from 2005 to 2014 (n=75671). We identified 20 low-value care measures 12 months prior to localized prostate cancer diagnosis using SEER-Medicare registry and claims files. Conservative management was defined as no treatment 0-12 months after diagnosis. Non cancer related total healthcare expenditures were defined as all costs independent of cancer treatment modalities 12-24 months after diagnosis.

Principal Findings: Overall, 25.2\% used low-value care services in 12 months prior to cancer diagnosis. Screening for asymptomatic carotid artery stenosis, Magnetic Resonance Imaging (MRI) for low-back pain, and traction for low-back pain were the most frequent low-value care procedures. Overall 18.1\% used CM and 
use of any low-value care was lower among patients using CM (15.2\%), with MRI for low-back pain, traction for low-back pain, and asymptomatic carotid artery stenosis more frequent among patients using treatment. In adjusted GLM models, every 1-unit increase in low-value care use was associated with an $\$ 858.82$ increase in healthcare expenditures. Multimorbidity (physical condition count), care fragmentation, and conservative management use were the top 3 predictors of healthcare expenditures with the highest absolute mean SHAP values.

Conclusions: We found incremental low-value healthcare use before incident prostate cancer diagnosis was significantly and positively associated with long-term non-cancer related costs. Using ML, both low-value care and CM use were highly ranked important features of cost prediction 12-24 months after incident prostate cancer diagnosis. 


\subsection{Introduction}

Low-value care, defined as unnecessary tests or treatment lacking a net clinical benefit to the patient, contribute to avoidable morbidities and excessive healthcare costs.(17) Low-value care is prevalent among elderly patients, affecting 1 in 4 Medicare beneficiaries(18) and accounting for $\$ 75.7-\$ 101.2$ billion in annual healthcare costs. $(19,20)$ Use of low-value healthcare services among medically complex patients can lead to cascades of unnecessary down-stream care, leading to costs as high as 10 times the original low-value healthcare service.(21)

Adults with incident localized prostate cancer represent a large, medically complex population at risk of low-value care in the form of overtreatment. Older adults with localized prostate cancer have high rates of multimorbidity and often experience care fragmentation that present barriers to shared decision making and evidence-based recommendations.(25) These adults may be at risk for receiving low-value care, augmenting down-stream costs. Many studies have examined comparative costs between different prostate cancer treatment modalities including conservative management (CM) (32); however, long term costs associated with overtreatment or low-value care remain unknown.

Recently, machine learning algorithms have been used to identify high-cost patients (98), including, for example, high-cost breast cancer patients (99). However, the potential predictive effects of low-value care on non-cancer related total healthcare expenditures among medically complex localized prostate cancer survivors remains little explored.

The primary objective of this paper is to examine whether low-value care is a leading predictor of longterm (12-24 months after diagnosis) non-cancer related expenditures among older adults with incident localized prostate cancer using machine learning algorithms and model agnostic interpretations. We used traditional generalized linear models to examine the association of life expectancy, high- and low-value care use, and care fragmentation on healthcare expenditures 12-24 months after the diagnosis of localized prostate cancer.

\subsection{Methods}

\section{Design}


This study used a retrospective longitudinal design with a 12-month baseline and a 24-month follow-up period. The baseline period comprised the 12-month period before date of localized prostate cancer diagnosis. The follow-up period included the 24-month after localized prostate cancer diagnosis. Healthcare expenditures were calculated during the second half of the follow-up period (12-24 months).

\section{Data sources}

This study used the Surveillance, Epidemiology, and End Results (SEER)-Medicare linked data files. SEER-Medicare data includes data on all incident prostate cancer cases occurring in 18 regions throughout the United States. Incident prostate cancer, prostate cancer specific clinical information (i.e., Gleason Score and Prostate-specific antigen values), and cancer staging information were derived from the SEER database. Medicare claims were derived from inpatient, outpatient, and other files linked to SEER.

Area Health Resource File (AHRF) files were linked via MEDPAR FIPS state and county codes and were used to calculate radiation oncologist and urologist densities. Census files were linked to calculate county-level median income quartiles.

\section{Study population}

Patients (age $\geq 66$ years) diagnosed with localized prostate cancer during the study period (2005-2014) were included in the study population. Cancer diagnosis was identified using International Classification of Diseases for Oncology (ICD-9). Localized prostate cancer was defined as cancer stage $\leq$ T2a.

Study inclusion/exclusion criteria:

Patients with localized prostate cancer were included in the study if alive and continuously enrolled in Medicare Parts A and B throughout the baseline and follow-up periods. Patients with missing cancer stage, PSA values, Gleason Scores were excluded. Patients diagnosed with prostate cancer at autopsy were excluded (Appendix Figure 7.7).

\section{Dependent variable/target}

Total healthcare expenditures were calculated by summing inpatient (MEDPAR), outpatient (Outsaf, $\mathrm{NCH}$ ), and "other" (durable medical equipment, and home health agency) claims. To compare non-cancer related healthcare expenditures, we subtracted treatment-related costs from total healthcare expenditures. All 
expenditures were estimated 12-24 months after localized prostate cancer diagnosis. Consumer price index (CPI) was used to adjust all expenditures to 2016 constant dollars. (100)

\section{Key Independent variables/features}

\section{Low-value care}

Various professional societies identify medically unnecessary and/or potentially harmful procedures, tests, and treatments for Choosing Wisely in an international effort to reduce low-value care.(20,22) Low-value healthcare services were operationalized using a claims-based algorithm representing Choosing Wisely campaign recommendations. The Low-value care algorithm included procedure, diagnosis, hospitalization, and BETOS codes using previously published methods.(6o-62)

\section{Other independent variables}

\section{Conservative management}

Conservative management was estimated within the first 12 months of the 24-month follow-up period using a validated claims-based algorithm.(53) Patients without treatment claims were designated as using conservative management.

\section{Multimorbidity}

The multimorbidity framework developed by the United States Department of Health and Human Services for guiding programs, practice, and policy guided the selection of chronic conditions as follows: arthritis, asthma, coronary artery disease, congestive heart failure, chronic kidney disease, chronic obstructive pulmonary disease, cardiac arrhythmias, acute myocardial infarction, dementia, diabetes, depression, hepatitis, hyperlipidemia, hypertension, human immunodeficiency virus, osteoporosis, substance abuse, schizophrenia, stroke, anemia, lower limb fracture.(55) We used clinically meaningful cut points (77) of multimorbidity (i.e., >2 chronic conditions) in descriptive analyses and included total counts of mental and physical health conditions in ML models.

\section{Prostate cancer comorbidity index}

Prostate cancer comorbidity index (PCCI) is a weighted comorbidity index validated in prostate cancer patient populations to predict 5 and 10 year life expectancy.(54) PCCI was calculated during the baseline period to estimate 5 and 10 year life expectancy. PCCI was categorized into 3 groups: 0-8 (> 10-year life expectancy); 9 
to 13 (5-10-year life expectancy); and > 13 (< 5-year life expectancy). In GLM models, PCCI total representing $>10$ years of life expectancy was used as the reference group. In ML models, we included PCCI as a continuous variable.

Managing pre-existing conditions and an incident prostate cancer diagnosis requires the use of limited patient, clinician, and practice ecosystem resources; patients must balance the management of several chronic conditions and participate in shared-decision making to select prostate cancer management within a practice environment with variation in supply and demand of healthcare resources (i.e., supply of clinician specialists). Therefore, a competing demands framework and previous literature reviews were used to guide the selection of other independent variables known to affect low-value care and prostate cancer treatment selection within patient, clinician, and practice ecosystem domains.(20,27,32,101)

Other independent variables included: diagnosis year group (2003-2009 and 2010-2013), low-risk prostate cancer (operationalized as Gleason Score $\leq 6$ and PSA test $\leq 10 \mathrm{ng} / \mathrm{mL}$ ), education (Less than highschool graduation and college graduation per 10,000 persons over age 65), income, urologist, and radiation oncologist quartiles (per 10,000 persons over age 65).

We used a modified version of the Bice-Boxerman continuity of care index to calculate care fragmentation.(56) The continuity of care index represents the concentration of visits per patient among health care providers based on visit number, proportion of encounters to each provider, and total number of visits. We used physician specialty codes representing primary care, oncology, and various specialist visit encounters; where $n$ is the total number of visits, $n k$ is the total number of visits associated with a physician specialty, and $k$ the total number of physician specialty codes.(59) For example, multiple encounters with a single provider would result in a score of zero, however, multiple encounters among several health care providers would result in a score approaching 1 . We included many specialties as patients with multimorbidity and/or limited life expectancy may require services from multiple specialists. Care fragmentation was measured during the 12 month baseline period.

All independent variables were calculated in the 12 months before cancer diagnosis.

\section{Statistical Analysis}


Chi-square tests and t-tests were used to identify significant group differences in low-value care and CM use by categorical variables. Generalized linear models (GLM) with log-link and gamma distribution were used to estimate total healthcare cost. GLM transforms the mean cost directly, reversing the transformation is possible without bias estimation of mean cost. Differences between cost associated with each independent variable of interest and the exponentiated intercept term were reported as the incremental average cost (i.e., mean) associated with independent variables of interest. GLMs were fit using separate unadjusted and adjusted regressions to identify independent and interactive associations of key independent variables and total healthcare expenditures. All statistical tests were 2-sided with a 5\% Type I error rate and were completed in SAS (SAS Institute Inc., Cary, NC, USA. Version 9.4, using Windows 10).

\section{Machine learning}

We used the XGBoost regression, a decision-tree ensemble machine learning algorithm, to determine the association of low-value care to healthcare expenditures.

Model training was conducted on $70 \%$ of the original data with 10 -fold cross validation based on the original data set. Hyperparameter adjustments were made during training interactions to address overfitting. Healthcare expenditures were log transformed to approximate a normal distribution. Prediction performance was assessed on unseen data by reserving a 30\% stratified random sample of the original data (i.e., the test set). Model tuning and test prediction performance were assessed using Residual Mean Squared Error and R2. Hyperparameter tuning parameters codes are available in Supplemental Materials 1.

SHAP is an additive feature attribution method that provides consistent, locally accurate, individualized feature attributions based on conditional expectation. SHAP is an improvement over other feature importance methods that utilize model performance metrics (i.e., gain/accuracy) subject to inconsistency bias. SHAP values can be sorted to illustrate feature importance and display cumulative effects of interactions. We used SHAP feature importance to describe the top 10 predictive features of non-cancer related total healthcare cost 12-24 months after incident prostate cancer diagnosis. SHAP summary and partial density plots for key variables were used to illustrate non-linear relationships between important features and noncancer related total healthcare cost. 
XGBoost hyperparameters can be adjusted to fine-tune regularization to control model complexity to optimize predictive performance.(43)

Machine learning analyses were conducted using Python 3.7 with open-source scikit-learn 0.21.3 wrapper interface for XGBoost regressor.

TreeSHAP was used to generate SHAP and SHAP feature plots using Python 3.7 and the SHAP package (0.29.2)

\subsection{Results}

\section{Study cohort description}

The study cohort was predominantly non-Hispanic white (81.3\%) (Table 4.1). The median age at diagnosis was 73.5 years and did not differ by year of diagnosis (2003-2009: $\mathrm{M}=73.8, \mathrm{SD}=5 \cdot 4 ;$ 2010-2013: $\mathrm{M}=$ 73.2, $\mathrm{SD}=5.2$ ). Overall 18.1\% used CM, defined as no curative treatment within 12 months of incident localized prostate cancer diagnosis.

Among older men with incident localized prostate cancer, 25.2\% used LVC in the 12 months prior to cancer diagnosis. Screening for asymptomatic carotid artery stenosis, Magnetic Resonance Imaging (MRI) for low-back pain, and traction for low-back pain were the most frequently reported low-value care procedures. Low-value care use counts ranged from 1 to 6 , with 13422, 4105, and 5649 patients using 1, 2, or 3 or more LVC services, respectively.

\section{Cohort characteristics and Low-value care}

Use of low-value care was significantly more common in older men aged 75 years or older, with multimorbidity, with less than 10 years of life expectancy, and who used preventative care (Table 4.1). Use of any low-value care was lower among patients using CM (15.2\%), with MRI for low-back pain, traction for lowback pain, and asymptomatic carotid artery stenosis more frequent among patients using treatment (Supplemental Table 4.2).

\section{Predictive feature importance of low valve care}

The predictive feature importance for low-value on healthcare expenditures ranked 7th (Figure 4.1). Absence of low-value care was inversely associated with healthcare expenditures, with a complex linear increase for every additional low-value care service used (Figure 4.2). 


\section{Statistical Associations of Low-value care to healthcare expenditures}

Average outpatient, inpatient, “other", and non-treatment related total healthcare expenditures were all significantly higher in low-value care than in no low-value care use groups (Table 4.2). In adjusted models using GLM with log-link function, every 1-unit increase in low-value care use was associated with an $\$ 858.82$ increase in healthcare expenditures (Table 4.4).

\section{Other Leading predictors of healthcare expenditures}

Multimorbidity (physical condition count), care fragmentation, and conservative management use were the top 3 predictors of healthcare expenditures with the highest absolute mean SHAP values (Figure 4.1). Partial density plots display linear and complex relationships with healthcare expenditures (Figure 4.2). Lower (o to 1) and higher (>99) values of care fragmentation were inversely associated with healthcare expenditure prediction (Figure 4.2). Physical health condition counts displayed more variation lower (o-4) versus higher (>7) chronic condition counts (Figure 4.2). In contrast, CM use was inversely associated with healthcare expenditure prediction (Figure 4.1).

\section{Statistical Associations among other important features}

Patients with a life expectancy of greater than 10 years had lower average healthcare expenditures $(\mathrm{M}=\$ 6941.96, \mathrm{SD}=16028.12)$ versus $5-10$ year $(\mathrm{M}=\$ 12638.58 \pm 24002.64)$ and less than 5 year $(M=\$ 21284.75 \pm 34633.97)$ life expectancy groups $(M=\$ 8893.27 \pm 19802.73)(F=1618.68, p<0.001)$. Healthcare expenditures were also significantly lower among patients using $\mathrm{CM}(\mathrm{M}=\$ 7332.74 \pm 18787.79)$ versus patients without CM use $(M=\$ 9237.09 \pm 20003.22)(\mathrm{p}<0.001)$.

CM use was associated with a \$932.05 decrease in non-treatment related total healthcare expenditures versus treatment for low risk prostate cancer (Table 4.4). Life expectancies less than 10 years were associated with significantly higher, and CM use, significantly lower, non-treatment related healthcare expenditures in adjusted GLM models (Table 4.3).

\subsection{Discussion}

In this study, we used claims-based algorithms supported by expert consensus to identify low-value care use prior to incident prostate cancer diagnosis. We observed that 1 in 4 older men with incident prostate cancer used low-value care prior to diagnosis and that low value care use was associated with higher healthcare 
expenditures, defined as total non-cancer related healthcare expenditures 12- 24 months after incident prostate cancer diagnosis.

Low-value care use prior to incident prostate cancer diagnosis was also associated with substantial increases in healthcare expenditures. On average, older patients using low-value care before an incident localized prostate cancer diagnosis were more likely to use treatment (versus CM) and to have higher healthcare expenditures. These associations remained robust after adjustment for prostate cancer-specific life expectancy estimates, low-risk prostate cancer, and practice ecosystem factors such as physician supply and care fragmentation, suggesting that low-value care was a significant driver of cost within 2 years of diagnosis.

Efforts to promote a high-value, patient-centered healthcare system has led to increased attention on excessive spending on low-value care, under-provision of high-value care, and policy approaches to controlling the rising cost of cancer care. Recently, the Centers for Medicare \& Medicaid Services (CMS) developed an Oncology Care First Model that establishes performance based payment components based on prospective total cost of care targets among patients completing curative treatment. As portions of cancer care reimbursement are transitioned from fee-for-service (FFS) to capitation payments, identifying the most important features of cost prediction could help providers establish more effective policies and interventions for high cost patients. Up-stream, low-value care, such as PSA screening for men over age 70, are known to contribute to increased short- and long- term costs associated with subsequent biopsies, treatment, and/or additional PSA tests. We found that other non-cancer related low-value care procedures were predictive of downstream expenditures after incident prostate cancer diagnosis. Our results have important implications for predicting patient cost after incident care diagnosis, given that low-value care use prior to incident prostate cancer diagnosis is predictive of higher cost.

CM use, defined as no treatment within one year post diagnosis (102), was one of the highest ranking features in predicting healthcare expenditures using machine learning models. Previous studies report that CM use, including both Active Surveillance and Watchful Waiting, could reduce annual healthcare costs associated with avoidable localized prostate cancer treatment by an estimated 1.2 billion dollars.(64) Other studies have reported 3 year median costs of \$1914 and \$10588 associated with CM versus low-value treatment, 
respectively.(32) We found that CM use was associated with a \$1905 reduction in 12-24 month healthcare expenditures independent of treatment-related costs.

High level evidence supports CM for localized prostate cancer among men with low-risk disease and/or limited life expectancy to reduce overtreatment morbidities and excessive healthcare expenditures. Regardless of initial treatment modality, incident prostate cancer patients encounter significant ephemeral anxiety that predisposes patients to treatment without prostate cancer specific mortality benefit.(94) Decisional conflict, adverse mental health effects, and survival expectations (103) associated with diagnosis are independent factors associated with treatment selection regardless of risk grouping or comorbidity burden.(27) Other studies report cascades of downstream care associated with avoidable treatment-related morbidities, competing demand for chronic condition management and preventative care, and compounding costs. (21) We speculate that an incident localized prostate cancer diagnosis among patients using low-value care services may affect other forms of overtreatment or use of unnecessary care leading to higher cancer survivorship costs.

Older adults with multimorbidity, living in an urban locality, and/or specialist dense regions are at risk for low-value care and down-stream cascades of high cumulative costs.(21) Patients with incident prostate cancer and multimorbidity are likely to encounter additional care fragmentation, which may include consultation from medical, urological, radiation, and/or surgical oncologists. Care fragmentation during prostate cancer survivorship is associated with redundant use of healthcare services, PSA testing, and proportional increases in cost.(24) As in previous studies, we found care fragmentation and multimorbidity prior to incident prostate cancer diagnosis to highly ranked predictors of long term healthcare expenditures.

Current Oncology Care Model (OCM) do not address care complexities associated with multimorbidity, care fragmentation, or non-treatment approaches to localized prostate cancer survivorship. Although multidisciplinary approaches have been proposed as a management strategy for prostate cancer survivors, it remains unclear how primary care and oncology professionals can effectively coordinate care in a fragmented FFS environment. More research is needed to identify predictors of high cost consumption of care in order to inform population health management initiatives and allow policymakers to develop tailored interventions to proactively address high-cost patients. 


\subsection{Strengths and Limitations}

Despite calls to identify low-value care use in vulnerable populations, no studies have investigated how use of low-value care in men with incident localized prostate cancer is associated with longer term survivorship costs. We used previously validated algorithms to identify conservative management use, prostate cancer specific mortality index, care fragmentation, and multimorbidity estimates to understand the predictive importance of low-value care use on healthcare cost during survivorship. We also estimated non-treatment related total expenditures 12-24 months after diagnosis to identify long-term costs lacking characterization in the current literature. We applied statistical, machine learning, and novel machine learning interpretative approaches to estimate and predict the impact of low-value care use on healthcare expenditures.

Our results must be interpreted with important limitations. First, this observational study is a claimsbased, retrospective analysis, therefore, our results are subject to unobservable variable bias and selection bias of paid Medicare claims. To minimize the proportion of missed claims we included beneficiairies only continously enrolled in Medicare (A\&B, without HMO) throughout the study period. Second, our analysis may not be generaliable to commerical insurance beneficiaries as we only observed Medicare FFS beneficiairies. Our estimates of low-value care use are likely underestimated as methods for identification of low value care in claims data are limited. Social determinants of health (i.e., income, education) were not available at the individual level therefore county level measures were used. We only included measures detectable within a one year period to match the length of the baseline period before diagnosis, likely leading to further underestimation of costs. Although NCCN guidelines recommend CM for patients with low-risk cancer and a life expectancy of less than 10 years, both curative therapies and Active Surveillance are evidenced-based choices for patients with a life expectancy of greater than 10 years.(6) Lastly, we did not account for follow-up costs such as PSA and/or biopsy procedures that are commonly part of routine medical practice for patients using Active Surveillance and/or treatment related monitoring.

\subsection{Conclusion}

In this study, incremental use of low-value healthcare showed a significant positive association with long-term, non-treatment related costs after adjustments for multiple possible confounders. Use of evidencebased CM after diagnosis was associated with lower non-treatment related total healthcare costs. Using 
machine learning, we estimate both low-value care and $\mathrm{CM}$ use to be high-ranking features of cost prediction 12-24 months after incident prostate cancer diagnosis. Targeting patients with low-value care use prior to cancer diagnosis using existing claims data could help to reduce low-value care related morbidities by proactively identifying high-cost patients. 
Table 4.1 Patient Characteristics by Low-value Care Use among Fee-for-Service Medicare Beneficiaries with Incident Localized Prostate Cancer using Linked SEER Cancer Registry, 2005-2014 (n=75671)

\begin{tabular}{|c|c|c|c|c|c|c|c|}
\hline & & \multicolumn{2}{|l|}{ LVC } & \multicolumn{2}{|l|}{ No LVC } & \multirow[b]{2}{*}{$\mathbf{X}^{2}$} & \multirow[b]{2}{*}{ p-value } \\
\hline & & $\mathbf{N}$ & $\%$ & $\mathbf{N}$ & $\%$ & & \\
\hline ALL & & 19,071 & 25.2 & 56,600 & $73 \cdot 3$ & & \\
\hline \multicolumn{2}{|c|}{ Age in Years } & & & & & 400.79 & $<0.001$ \\
\hline & $66-74$ & 12075 & 24.4 & 37474 & 75.6 & & \\
\hline & $75^{+}$ & 8135 & 31.1 & 17987 & 68.9 & & \\
\hline \multicolumn{2}{|c|}{ Race } & & & & & 39.00 & $<0.001$ \\
\hline & White & 16701 & 27.1 & 44853 & 72.9 & & \\
\hline & Black & 2079 & 24.1 & 6533 & 75.9 & & \\
\hline & Hispanic & 352 & 27.5 & 927 & 72.5 & & \\
\hline & Other & 1025 & 25.4 & 3014 & 74.6 & & \\
\hline \multicolumn{2}{|c|}{ Marital Status } & & & & & 52.97 & $<0.001$ \\
\hline & Married & 1271 & 24.7 & 3881 & $75 \cdot 3$ & & \\
\hline & Unmarried & 13908 & 26.5 & 38585 & 73.5 & & \\
\hline & Separated/Divorced/Widowed & 2495 & 26.3 & 7002 & 73.7 & & \\
\hline & Unknown & 2536 & 29.7 & 5993 & 70.3 & & \\
\hline \multicolumn{2}{|c|}{ Income quintiles } & & & & & 38.85 & $<0.001$ \\
\hline & First & 3746 & 25.5 & 10938 & 74.5 & & \\
\hline & Second & 3841 & 26.0 & 10912 & 74.0 & & \\
\hline & Third & 3909 & 26.5 & 10860 & 73.5 & & \\
\hline & Four & 3992 & 27.1 & 10726 & 72.9 & & \\
\hline & Fifth & 4283 & 28.4 & 10785 & 71.6 & & \\
\hline \multicolumn{2}{|c|}{ Education quintiles } & & & & & 51.10 & $<0.001$ \\
\hline & First & 4184 & 28.4 & 10545 & 71.6 & & \\
\hline & Second & 4062 & 27.4 & 10748 & 72.6 & & \\
\hline & Third & 3962 & 26.7 & 10890 & $73 \cdot 3$ & & \\
\hline & Four & 3872 & 26.1 & 10941 & 73.9 & & \\
\hline & Fifth & 3712 & 25.0 & 11146 & 75.0 & & \\
\hline \multicolumn{2}{|c|}{ Urologist density quintiles } & & & & & 49.73 & $<0.001$ \\
\hline & First & 3809 & $25 \cdot 3$ & 11262 & 74.7 & & \\
\hline & Second & 4265 & 27.7 & 11114 & 72.3 & & \\
\hline & Third & 3889 & 26.9 & 10562 & 73.1 & & \\
\hline & Four & 4349 & 28.0 & 11206 & 72.0 & & \\
\hline & Fifth & 3880 & 25.7 & 11231 & 74.3 & & \\
\hline
\end{tabular}

Note: Based on 75671 older (age $\geq 66$ years) Fee-for-Service Medicare beneficiaries, with continuous enrollment in Medicare part A \& Part B, diagnosed with incident localized prostate cancer between 2005 and 2014. SEER = Surveillance, Epidemiology and End Results cancer Registry, CM= Conservative management. 


\begin{tabular}{|c|c|c|c|c|c|c|}
\hline \multicolumn{7}{|l|}{ continued Table 4.1} \\
\hline Radiation oncologist quintiles & & & & & 202.91 & $\mid<0.001$ \\
\hline First & 3773 & 24.5 & 11641 & $75 \cdot 5$ & & \\
\hline Second & 4239 & 29.0 & 10365 & 71.0 & & \\
\hline Third & 3739 & 25.2 & 11071 & 74.8 & & \\
\hline Four & 4667 & 29.9 & 10960 & 70.1 & & \\
\hline Multimorbidity & & & & & 6466.74 & $<0.001$ \\
\hline o-2 Chronic conditions & 4052 & 12.1 & 29314 & 87.9 & & \\
\hline$>2$ Chronic conditions & 16158 & 38.2 & 26147 & 61.8 & & \\
\hline Prostate Cancer Comorbidity Index & & & & & 5992.61 & $<0.001$ \\
\hline 10 or more years life expectancy & 11676 & 20.2 & 46207 & 79.8 & & \\
\hline 5-10 years life expectancy & 5290 & 42.6 & 7140 & 57.4 & & \\
\hline 5 or less years life expectancy & 3244 & 60.5 & 2114 & 39.5 & & \\
\hline Conservative management & & & & & 152.88 & $<0.001$ \\
\hline Conservative management use & 3070 & 22.5 & 10592 & $77 \cdot 5$ & & \\
\hline No conservative management use & 17140 & 27.6 & 44869 & 72.4 & & \\
\hline Preventative A1c test & & & & & 497.32 & $<0.001$ \\
\hline A1c test & 3604 & $35 \cdot 9$ & 6442 & 64.1 & & \\
\hline No a1c test & 16606 & $25 \cdot 3$ & 49019 & 74.7 & & \\
\hline Preventative flu & & & & & 644.46 & $<0.001$ \\
\hline Influenza vaccination & 9630 & 31.7 & 20756 & 68.3 & & \\
\hline No influenza vaccination & 10580 & 23.4 & 34705 & 76.6 & & \\
\hline Preventative lipid screen & & & & & 146.13 & $<0.001$ \\
\hline Lipid test & 6270 & 30.2 & 14520 & 69.8 & & \\
\hline No lipid test & 13940 & 25.4 & 40941 & 74.6 & & \\
\hline Metro & & & & & 60.93 & $<0.001$ \\
\hline Metro county & 17337 & 27.2 & 46301 & 72.8 & & \\
\hline Non-metro county & 2855 & 23.9 & 9074 & 76.1 & & \\
\hline SEER Region & & & & & 115.28 & $<0.001$ \\
\hline Northeast & 4499 & 29.0 & 11020 & 71.0 & & \\
\hline South & 5018 & 25.9 & 14345 & 74.1 & & \\
\hline North Central & 2533 & 29.6 & 6030 & 70.4 & & \\
\hline West & 8160 & 25.3 & 24066 & 74.7 & & \\
\hline \begin{tabular}{|l|} 
Year of diagnosis \\
\end{tabular} & & & & & 166.45 & $<0.001$ \\
\hline $\mid<2009$ & 9651 & 29.1 & 23567 & 70.9 & & \\
\hline$\geq 2009$ & 10559 & 24.9 & 31894 & 75.1 & & \\
\hline
\end{tabular}

Note: Based on 75671 older (age $\geq 66$ years) Fee-for-Service Medicare beneficiaries, with continuous enrollment in Medicare part A \& Part B, diagnosed with incident localized prostate cancer between 2005 and 2014. SEER= Surveillance, Epidemiology and End Results cancer Registry, CM= Conservative management. 
Table 4.2 Healthcare Expenditures by Low-value Care Use among Elderly Fee-for-Service Medicare Beneficiaries with Incident Localized Prostate Cancer SEER-Medicare, 2005-2014 (n=75671)

\begin{tabular}{|l|c|c|c|c|}
\hline & LVC & No LVC & & \\
\hline & $\mathbf{n = 1 9 0 7 1}$ & $\mathbf{n = 5 6 6 0 0}$ & & \\
\hline Total healthcare expenditures & Mean (SD) & Mean (SD) & CI & p-value \\
\hline Outpatient expenditures & $6467.20(8740.10)$ & $4063.00(6110.23)$ & $4619.42,4718.42$ & $<0.001$ \\
\hline Inpatient expenditures & $5276.75(18032.29)$ & $3114.84(13664.18)$ & $3553.42,3765.97$ & $<0.001$ \\
\hline Other expenditures & $845.69(2996.22)$ & $469.98(2120.18)$ & $547.74,803.17$ & $<0.001$ \\
\hline
\end{tabular}

Note: Based on 75671 older (age $>66$ years) Fee-for-Service Medicare beneficiaries, with continuous enrollment in Medicare part A \& Part B, diagnosed with incident localized prostate cancer between 2005 and 2014.

Healthcare expenditures for patients completing curative treatment (i.e., "No CM") do not include treatment costs. $\mathrm{CI}=$ Confidence interval, SEER = Surveillance, Epidemiology and End Results cancer Registry, CM= Conservative management. 
Table 4.3. Healthcare Expenditures by Conservative Management Use among Elderly Fee-forService Medicare Beneficiaries with Incident Localized Prostate Cancer SEER-Medicare, 2005-2014 $(\mathrm{n}=75671)$

\begin{tabular}{|l|c|c|c|}
\hline & CM & No CM & \\
\hline & $\mathbf{n = 1 3 6 6 2}$ & $\mathbf{n = 6 2 0 0 9}$ & \\
\hline Total healthcare expenditures & Mean (SD) & Mean (SD) & $\mathrm{p}$-value \\
\hline Outpatient expenditures & $7332.74(18787.79)$ & $9237.09(20003.22)$ & $<0.001$ \\
\hline Inpatient expenditures & $3681.65(7334.92)$ & $4886.44(6840.01)$ & $<0.001$ \\
\hline Other expenditures & $539.8(2438.32)$ & $570.16(2363.56)$ & \\
\hline
\end{tabular}

Note: Based on 75671 older (age $>66$ years) Fee-for-Service Medicare beneficiaries, with continuous enrollment in Medicare part A \& Part B, diagnosed with incident localized prostate cancer between 2005 and 2014. Healthcare expenditures for patients completing curative treatment (i.e., "No CM") do not include treatment costs. $\mathrm{CI}=$ Confidence interval, SEER= Surveillance, Epidemiology and End Results cancer Registry, CM= Conservative management. 
Table 4.4 Parameter estimates of PCCI categories from unadjusted and adjusted generalized linear models on 12-24 months healthcare expenditures among elderly Medicare fee-for-service beneficiaries with incident localized prostate cancer diagnosis using SEER-Medicare data, 2005-2015 ( $\mathrm{n}=75671)$.

\begin{tabular}{|c|c|c|c|c|}
\hline & $\begin{array}{c}\text { Parameter } \\
\text { Estimate (SE) }\end{array}$ & Change \# & $\begin{array}{c}\text { Parameter } \\
\text { Estimate (SE) }\end{array}$ & Change \# \\
\hline & \multicolumn{2}{|c|}{ Unadjusted GLM } & \multicolumn{2}{|c|}{ Adjusted GLM } \\
\hline \multicolumn{5}{|l|}{ Age } \\
\hline $66-74$ & $8.949(0.010)$ & 7700.19 & $8.416(0.055)$ & $\$ 4518.79$ \\
\hline 75 and over & $0.370(0.017)$ & 3447.64 & $0.247(0.018)$ & $\$ 1266.07$ \\
\hline \multicolumn{5}{|l|}{$\begin{array}{l}\text { Prostate cancer } \\
\text { comorbidity score }\end{array}$} \\
\hline 10 or more years & $8.845(0.009)$ & 6939.60 & $8.416(0.055)$ & $\$ 4518.79$ \\
\hline Less than 5 years & $1.120(0.031)$ & 14329.27 & $0.895(0.033)$ & $\$ 6540.21$ \\
\hline Between 5 and 10 years & $.599(0.022)$ & 5692.54 & $0.470(0.023)$ & $\$ 2711.25$ \\
\hline \multicolumn{5}{|l|}{ Conservative management } \\
\hline $\mathrm{No}$ & 9.131 (0.009) & 7331.97 & $8.416(0.055)$ & $\$ 4518.79$ \\
\hline Yes & $-0.231(0.021)$ & -1905.28 & $-0.219(0.022)$ & $\$-932.05$ \\
\hline \multicolumn{5}{|l|}{ Low-value care ${ }^{1}$} \\
\hline & $8.956(0.009)$ & 7754.28 & $8.416(0.055)$ & $\$ 4518.79$ \\
\hline Total Low-value care & $0.312(0.013)$ & 2839.27 & $0.174(0.012)$ & $\$ 858.82$ \\
\hline \multicolumn{5}{|l|}{ Low-risk prostate cancer } \\
\hline $\mathrm{No}$ & 9.141 (0.010) & 9320.77 & $8.416(0.055)$ & $\$ 4518.79$ \\
\hline Yes & $-.140(0.017)$ & -1217.68 & $-0.023(0.018)$ & NS \\
\hline \multicolumn{5}{|l|}{ Care fragmentation } \\
\hline & $.010(0.000)$ & 52.87 & .006 (0.00) & $\$ 27.19$ \\
\hline
\end{tabular}

Note: Based on 75671 older (age $>66$ years) Fee-for-Service Medicare beneficiaries, with continuous enrollment in Medicare part A \& Part B, diagnosed with incident localized prostate cancer between 2005 and 2014. Healthcare expenditures for patients completing curative treatment (i.e., "No CM") do not include treatment costs. Total healthcare expenditures include inpatient, outpatient, durable medical equipment, and home health agency costs with treatment-related cost subtracted.

1. Sum of low-value care procedures in 12 months before prostate cancer diagnosis.

Change \# was calculated by difference between the 1) exponentiation of the model intercept term and 2) the sum of the intercept and the variable parameter estimate.

Compare to omitted category

$\mathrm{SE}=$ Standard Error, SEER= Surveillance, Epidemiology and End Results cancer Registry, CM= Conservative management. 
Figure 4.1 SHAP Feature Importance and Summary Plot of Low-value Care and Conservative Management Use on Non-Treatment Related Total Healthcare Expenditures among Fee-for-Service Medicare Beneficiaries with Incident Localized Prostate Cancer using Linked SEER Cancer Registry, 2005-2014 (n=75671)

A.

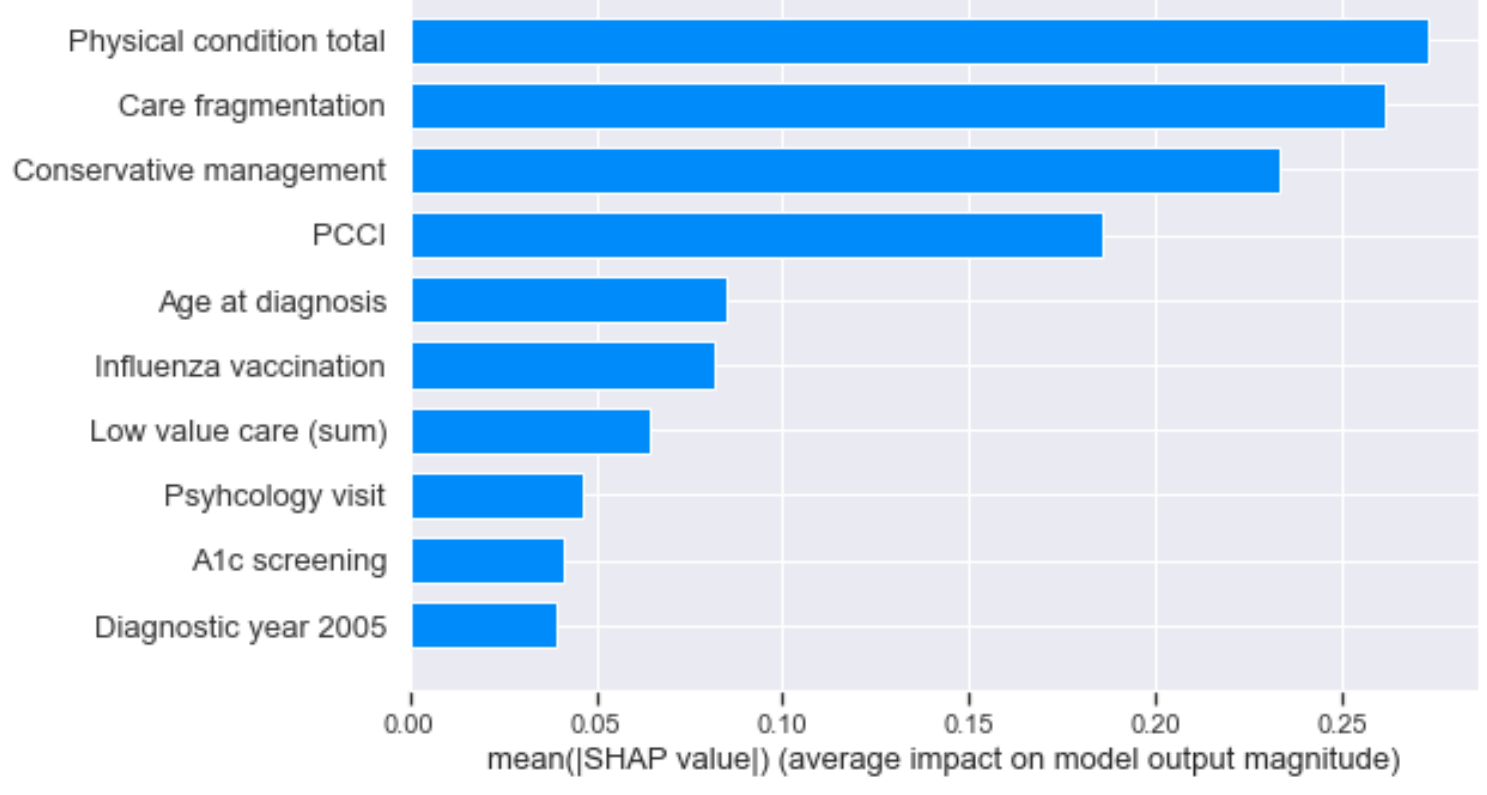

B.

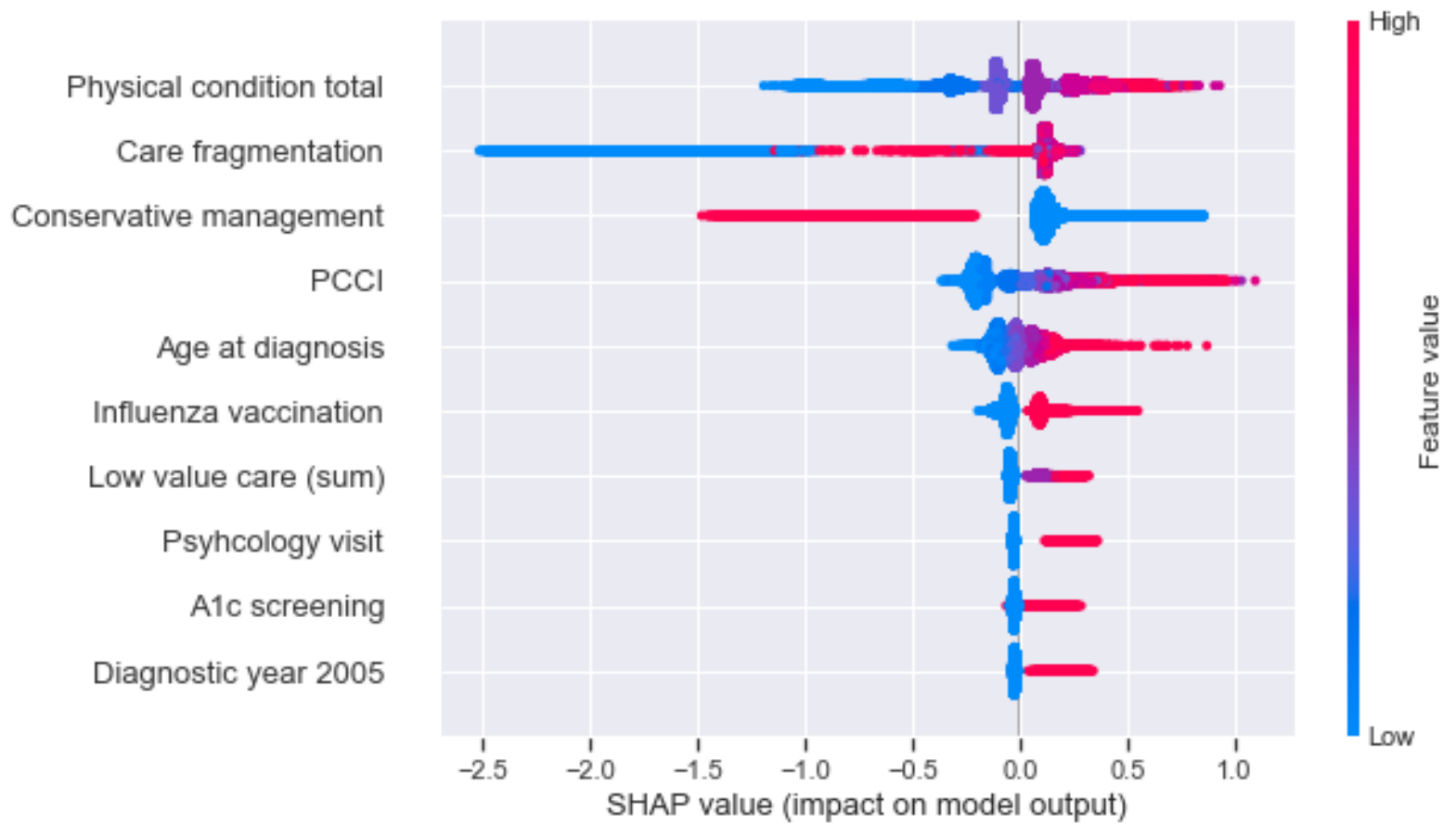

(A) Mean SHAP values and (B) SHAP summary plot in descending order of log-dollars. Based on 75671 older (age $\geq 66$ years) Fee-for-Service Medicare beneficiaries, with continuous enrollment in Medicare Part A \& Part B, diagnosed with incident localized prostate cancer (Stage $\leq$ T2a) between 2005 and 2014. 
Figure 4.2 SHAP Partial Density Plots of Selected Features on Non-Treatment Related Total Healthcare

Expenditures among Fee-for-Service Medicare Beneficiaries with Incident Localized Prostate Cancer using Linked SEER Cancer Registry, 2005-2014 (n=75671)
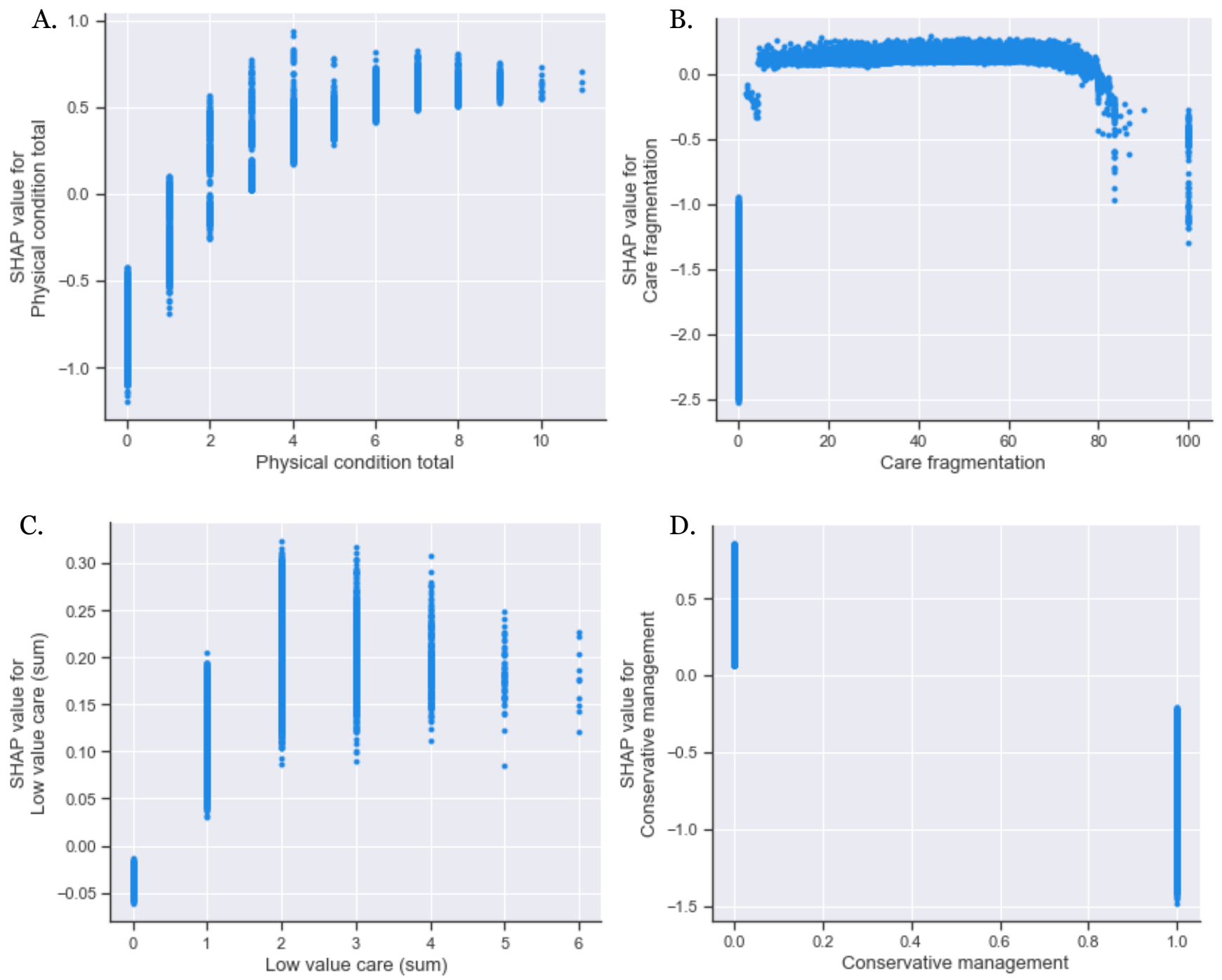

SHAP Partial Dependence plots (PDP) of SHAP values (log-dollars) values by (A.) Multimorbidity (Physical conditions only), (B.) Care fragmentation, (C) Low value care total prior to incident prostate cancer diagnosis, and (D.) Conservative Management. Based on 75671 older (age $\geq 66$ years) Fee-for-Service Medicare beneficiaries, with continuous enrollment in Medicare Part A \& Part B, diagnosed with incident localized prostate cancer (Stage $\leq$ T2a) between 2005 and 2014.

Physical condition total $=$ Total count of multimorbidity physical conditions.

Care fragmentation = Bice-Boxerman continuity of care index to calculate care fragmentation during the 12 month baseline period (See Methods).

Low value care $=$ Sum of low value care prior to incident prostate cancer diagnosis.

Conservative Management $=$ Yes (1)/No (o).

SHAP = Shapley Additive ePlanations.

SEER = Surveillance, Epidemiology, and End Results cancer Registry. 


\section{Chapter 5}

\section{Summary and Conclusion}

\subsection{Summary of Findings and Discussion}

The goal of this dissertation is to highlight healthcare practices that provide limited clinical benefit, potentially harmful effects, and a significant economic burden among older men with incident localized prostate cancer. This dissertation also highlights the role of patient experiences in receiving high value care for incident prostate cancer. Older men with localized prostate cancer represent a large, medically complex and growing population. Although prostate cancer is heterogeneous at the population level, high-level randomized evidence concluded a lack of significant survival benefit of treatment (surgery, radiation, chemotherapy, hormone therapy, cryotherapy) among men with intermediate grade disease and 15 year life expectancy.(104106) Conservative management (Active Surveillance and Watchful Waiting) within the context of cancer risk group, life expectancy estimates, and personal values has been promoted as high-value care as significant quality decrement and high costs of overtreatment can be avoided. Despite evidence-based guidelines recommending conservative management for a decade, many men with low-risk prostate cancer and limited life expectancy received treatment for prostate cancer. Higher rates of treatment use among fee-for-service (58\%) versus integrated healthcare beneficiaries (20\%) highlight disparities in evidenced-based healthcare delivery.

This dissertation focused on three related studies: 1) examine the leading predictors of low-value healthcare practice (i.e., prostate cancer treatment for low-risk prostate cancer) among older men; 2) assess the role of patient-reported experience with care on high-value prostate cancer management; and 3) Estimate the association of high-value care on non-cancer related healthcare expenditures. In all of these studies an emphasis is placed on multimorbidity because of high prevalence and negative clinical, humanistic, and economic effects throughout cancer survivorship. We specifically examined the associations of patient-reported experiences with evidence-based treatment selection and low-value care to reflect the standards of the Institute of Medicines (IOM) and The National Quality Task Force aim to promote patient-centered care and reductions in inappropriate, wasteful healthcare practices among adults managing chronic conditions. $(15,16)$ 
This dissertation filled a knowledge gap through a comprehensive analysis of clinical and non-clinical factors that drive low-value cancer treatment among incident low-risk prostate cancer. A novel feature of this dissertation is the use of machine learning algorithms and the application of model agnostic interpretable machine learning techniques. This dissertation also made a unique contribution and clarified the role of life expectancy on the complex decision making process of choosing from several treatments (surgery, radiation, cryotherapy, and or chemotherapy and hormone therapy) or conservative management. We distinguished between comorbidity and life expectancy to estimate the independent contribution of life expectancy on treatment choice. This dissertation is a series of firsts for its measurement of care fragmentation during cancer survivorship, use of machine learning algorithms and unlocking the "black box" prediction with interpretable machine learning techniques, and assessing the role of patient-reported experiences on high-value care among older men with incident prostate cancer.

\subsection{Implications and Suggestions for Future Research}

A common theme that emerged from our studies is the negative effect of multimorbidity on outcomes (i.e., low-value cancer treatment, high-value conservative management, and non-cancer related healthcare expenditures). This finding is not surprising given the voluminous data and robust evidence on the negative health effects of multimorbidity on health outcomes in a variety of settings and populations. Adults with multimorbidity and incident cancer are at high-risk for poor quality of both cancer and non-cancer care $(37,107)$ as current non-cancer clinical guidelines are developed within a single-disease framework.(108) Men

with multimorbidity and incident cancer may not opt for conservative management because of a preference for immediate cure (ie, "take care of it")(87) and may have a preference to avoid one more condition that requires long-term management. In addition, men are likely to experience fear of non-treatment regret,(88) emotional distress,(89) and anxiety.(90) The implementation of OCM which provides a bundled payment for all care during an episodic treatment will inherit the complex management requirements of men with multimorbidity and incident prostate cancer. Value-based payment mechanisms are needed to support the conservative management of incident localized prostate cancer with multimorbidity, especially among men with limited life expectancy, who benefit more from non-cancer chronic condition management versus curative treatment and 
other potential cascades of low-value care or increased care fragmentation without the benefit of reduced prostate cancer specific mortality.

Our results suggest focusing on reducing care fragmentation, such as broader system reforms to increase care continuity between primary care physicians and oncologists, could help reduce treatment selection among patients with low-risk prostate cancer. Considering our results, we speculate that care fragmentation is likely to contribute to differing treatment rates for low-risk prostate cancer between Medicare FFS and Veterans Administration beneficiaries (approximately 37\%).(46)

For a subset of individuals older men with localized prostate cancer we observed that patient experiences with timely care was important in treatment choice. Timely access to care for men with localized prostate cancer is not limited to initial diagnosis of prostate cancer, but the opportunity and ease by which a patient is able to access and utilize needed services along the continuum of care throughout survivorship.(93) Choices for elderly localized prostate cancer patients involve selecting curative and non-curative treatments with trade-offs in efficacy, potential adverse quality of life effects, and competing risk mortality. Timeliness of care domains used in this dissertation, such as perceived barriers to appointment scheduling, are fundamental to shared decision-making among multiple health-care providers that significantly influence treatment choice for men with localized prostate cancer.(94-96) We speculate that adults with higher timely care ratings may choose CM if they have a favorable perception of health-care system capacity to provide services once a need is detected. Our results suggest that addressing specific modifiable barriers to timely care along the cancer continuum for older adults with localized prostate cancer and limited life expectancy could reduce the adverse effects of overtreatment on health outcomes and costs.

A noteworthy finding is the superior performance of machine learning algorithms over traditional statistical models, specifically logistic regression. This dissertation confirmed flexibility, predictive accuracy, and ability of machine algorithms to handle multicollinear variables. With machine learning, model misspecification can be avoided through the use of cross-validation model tuning on "training" data before applying the algorithm to a "test" or new data set. Once validated, interpretable machine learning approaches, such as SHAP, can be applied to reveal the complexity and non-linearity of associations using feature importance and SHAP summary and partial density plots. In our dissertation, machine learning analyses 
revealed significant variation among individuals (i.e. patient-level) of estimated outcomes at specific feature values. Our results highlight the need to target individuals with non-linear, complex feature combinations pushing them toward low-value outcomes that are otherwise unobservable with "on average" traditional statistical approaches.

The application of machine learning algorithms to predict low- and high- value care are in alignment with the National Quality Forum created a Technology Evaluation Framework.(16) These organizations promote the use of advanced technologies, such as machine learning algorithms, to identify solutions to address high-value healthcare practices. The framework seeks to strengthen health literacy to complement shared decision making processes to facilitate evidence-based medicine while reducing propensity for lowvalue services. Our study demonstrates that machine learning and interpretive approaches can be leveraged to proactively identify patients who may benefit from interventions, for both patients and physicians, to facilitate shared decision making regarding prostate cancer care and reduce the use of low-value care.

Our results from the analysis on the association of low- and high-value care to non-cancer related healthcare expenditures suggest the development and implementation of targeted payment reforms could reduce the economic burden to the payers, specifically Medicare. Current Oncology Care Model (OCM) are voluntary five-year bundled payment programs developed by the Center for Medicare \& Medicaid Innovation (CMMI) to facilitate high-value, lower-cost healthcare through improved care coordination and episodic or bundled reimbursement. OCM incentivizes providers to lower the total cost of care for patients throughout the treatment episodes.(109) Although multidisciplinary approaches have been proposed as a management strategy for prostate cancer survivors, it remains unclear how primary care and oncology specialist delivery coordinated care in a fragmented FFS environment. Our research highlights how the application of machine learning can be used to identify patients at risk of low-value care. Healthcare entities can leverage large patient data sets, claims registries, and electronic medical records to continuously re-train machine learning models to improve predictive accuracy and provide personalized medicine for many diseases. However, the implementation of such technologies will require a robust technical investment from healthcare entities to develop specific algorithms for the patient population they serve. Policy makers should consider mechanisms 
to support healthcare organizations in the development of predictive "precision" population health technologies.

\subsection{Strengths and Limitations}

Our dissertation was a series of many first. We used both multimorbidity and prostate cancer-specific of life expectancy measures to understand the independent contributions of comorbidity and prostate cancer specific mortality on treatment selection among men with localized prostate cancer. We used robust machine learning and statistical methods to explore novel predictive and statistical associations of low-value care, patient-reported experience measures, and care fragmentation on overtreatment and cost outcomes among older adults with multimorbidity. Our research supports nationally recognized strategic objectives for improving patient-centered care while identifying predictors to reduce inappropriate low-value care. We used cancer registry, claims, MCAHPS surveys, and other socio-demographic data linkages to incorporate measures of multimorbidity, care fragmentation, patient-reported experience measures, social determinants of health, and low-value care in machine learning and statistical models. Although educational attainment may not be modifiable among older adults, initiatives such as "health in all policies" by the World Health Organization and the Centers for Disease and Prevention Control suggest the integration and consideration of community health policy(73) as social, economic, and physical environments have a significant impact on the health of an individual. With these considerations, we developed machine learning and statistical approaches using a competing demands framework in all of our study aims.

Due to the retrospective nature of this work our findings are not causal. Generalizability to younger patients, health Maintenance Organization, and integrated healthcare networks may not be appropriate as our studies included only fee-for-service beneficiaries. Measurement of low-value care in claims data requires the estimation of clinical scenarios using inclusion and exclusion criteria that are diagnosis and/or procedure specific, and often include a temporal component. We could only select procedures meeting criteria for lowvalue care that could be operationalized accurately in claims data, therefore, we are likely to underestimate the burden of low-value care. 


\subsection{Conclusion}

Our studies reveal that low-value cancer treatment is highly-prevalent as only 2 in 5 men received conservative management. In predictive models of low-value care (i.e., overtreatment) and high-value care (i.e., conservative management) we did not find a life expectancy of 10 or more years to be predictive. These results indicate that treatment choice among older adults, within the context of life expectancy, do not reflect evidence-based guidelines for prostate cancer treatment. Our findings suggest a need for broad implementation of interventions to improve patient and physician education during the shared-decision process. 


\section{References}

1. Siegel RL, Miller KD, Fuchs HE, Jemal A. Cancer Statistics, 2021. CA Cancer J Clin. 2021 Jan 12;71(1):7-33.

2. Siegel RL, Miller KD, Jemal A. Cancer statistics, 2018. CA Cancer J Clin. 2018 Jan 4;68(1):7-30.

3. Negoita S, Feuer EJ, Mariotto A, Cronin KA, Petkov VI, Hussey SK, et al. Annual Report to the Nation on the Status of Cancer, part II: Recent changes in prostate cancer trends and disease characteristics. Cancer. 2018 Jul 1;124(13):2801-2814.

4. Amin MB, Edge SB, Greene FL, Byrd DR, Brookland RK, Washington MK, et al., editors. AJCC cancer staging manual. Cham: Springer International Publishing; 2017.

5. Sanda MG, Cadeddu JA, Kirkby E, Chen RC, Crispino T, Fontanarosa J, et al. Clinically localized prostate cancer: AUA/ASTRO/SUO guideline. part I: risk stratification, shared decision making, and care options. J Urol. 2018;199(3):683-690.

6. National Comprehensive Cancer Network. NCCN Clinical Practice Guidelines in Oncology (NCCN Guidelines) Prostate Cancer Version 2.2020 [Internet]. 2020 [cited 2020 May 25]. Available from: https://www.nccn.org/professionals/physician_gls/pdf/prostate.pdf

7. Tosoian JJ, Mamawala M, Epstein JI, Landis P, Wolf S, Trock BJ, et al. Intermediate and Longer-Term Outcomes From a Prospective Active-Surveillance Program for Favorable-Risk Prostate Cancer. J Clin Oncol. 2015 Oct 20;33(30):3379-3385.

8. Klotz L, Vesprini D, Sethukavalan P, Jethava V, Zhang L, Jain S, et al. Long-term follow-up of a large active surveillance cohort of patients with prostate cancer. J Clin Oncol. 2015 Jan 20;33(3):272-277.

9. Donovan JL, Hamdy FC, Lane JA, Mason M, Metcalfe C, Walsh E, et al. Patient-Reported Outcomes after Monitoring, Surgery, or Radiotherapy for Prostate Cancer. N Engl J Med. 2016 Oct 13;375(15):1425-1437.

10. Chen RC, Basak R, Meyer A-M, Kuo T-M, Carpenter WR, Agans RP, et al. Association Between Choice of Radical Prostatectomy, External Beam Radiotherapy, Brachytherapy, or Active Surveillance and Patient-Reported Quality of Life Among Men With Localized Prostate Cancer. JAMA. 2017 Mar 21;317(11):1141-1150.

11. Barocas DA, Alvarez J, Resnick MJ, Koyama T, Hoffman KE, Tyson MD, et al. Association Between Radiation Therapy, Surgery, or Observation for Localized Prostate Cancer and Patient-Reported Outcomes After 3 Years. JAMA. 2017 Mar 21;317(11):1126-1140.

12. Chen RC, Rumble RB, Loblaw DA, Finelli A, Ehdaie B, Cooperberg MR, et al. Active surveillance for the management of localized prostate cancer (Cancer Care Ontario Guideline): American Society of Clinical Oncology clinical practice guideline endorsement. J Clin Oncol. 2016 Jun 20;34(18):2182-2190.

13. Mohler JL. The 2010 NCCN clinical practice guidelines in oncology on prostate cancer. J Natl Compr Canc Netw. 2010 Feb;8(2):145.

14. Sampurno F, Zheng J, Di Stefano L, Millar JL, Foster C, Fuedea F, et al. Quality indicators for global benchmarking of localized prostate cancer management. J Urol. 2018 Mar 1;200(2):319-326.

15. Institute of Medicine (US) Committee on Quality of Health Care in America. Executive Summary Crossing the Quality Chasm - NCBI Bookshelf. 2001;

16. Agrawal S, Kizer K. The Care We Need - Driving Better Outcomes For People And Communities. The National Quality Forum; 2020.

17. Emanuel EJ, Fuchs VR. The perfect storm of overutilization. JAMA. 2008 Jun 18;299(23):2789-2791.

18. Schwartz AL, Landon BE, Elshaug AG, Chernew ME, McWilliams JM. Measuring low-value care in Medicare. JAMA Intern Med. 2014 Jul;174(7):1067-1076.

19. Shrank WH, Rogstad TL, Parekh N. Waste in the US health care system: estimated costs and potential for savings. JAMA. 2019 Oct 15;322(15):1501-1509. 
20. Colla CH, Morden NE, Sequist TD, Schpero WL, Rosenthal MB. Choosing wisely: prevalence and correlates of low-value health care services in the United States. J Gen Intern Med. 2015 Feb;30(2):221228.

21. Ganguli I, Lupo C, Mainor AJ, Raymond S, Wang Q, Orav EJ, et al. Prevalence and Cost of Care Cascades After Low-Value Preoperative Electrocardiogram for Cataract Surgery in Fee-for-Service Medicare Beneficiaries. JAMA Intern Med. 2019 Jun 3;

22. American Board of Internal Medicine. Choosing Wisely. An initiative of the ABIM foundation. [Internet]. ABIM Foundation. 2020 [cited 2020 Nov 9]. Available from: https://www.choosingwisely.org/

23. Baxi SS, Kale M, Keyhani S, Roman BR, Yang A, Derosa AP, et al. Overuse of health care services in the management of cancer: A systematic review. Med Care. 2017;55(7):723-733.

24. Skolarus TA, Zhang Y, Hollenbeck BK. Understanding fragmentation of prostate cancer survivorship care: implications for cost and quality. Cancer. 2012 Jun 1;118(11):2837-2845.

25. Garg T, Young AJ, Kost KA, Danella JF, Larson S, Nielsen ME, et al. Burden of Multiple Chronic Conditions among Patients with Urological Cancer. J Urol. 2018;199(2):543-550.

26. Frandsen BR, Joynt KE, Rebitzer JB, Jha AK. Care fragmentation, quality, and costs among chronically ill patients. Am J Manag Care. 2015 May;21(5):355-362.

27. Kinsella N, Stattin P, Cahill D, Brown C, Bill-Axelson A, Bratt O, et al. Factors Influencing Men's Choice of and Adherence to Active Surveillance for Low-risk Prostate Cancer: A Mixed-method Systematic Review. Eur Urol. 2018 Mar 26;74(3):261-280.

28. Schmidt T, Valuck T, Perkins B, Riposo J, Patel P, Westrich K, et al. Improving patient-reported measures in oncology: a payer call to action. J Manag Care Spec Pharm. 2021 Jan;27(1):118-126.

29. Doyle C, Lennox L, Bell D. A systematic review of evidence on the links between patient experience and clinical safety and effectiveness. BMJ Open. 2013 Jan 3;3(1).

30. Levit L, Balogh E, Nass S, Ganz PA, Committee on Improving the Quality of Cancer Care: Addressing the Challenges of an Aging Population, Board on Health Care Services, et al. Patient-Centered Communication and Shared Decision Making - Delivering High-Quality Cancer Care - NCBI Bookshelf. 2013 Dec 27;

31. Mollica MA, Enewold LR, Lines LM, Halpern MT, Schumacher JR, Hays RD, et al. Examining colorectal cancer survivors' surveillance patterns and experiences of care: a SEER-CAHPS study. Cancer Causes Control. 2017 Oct;28(10):1133-1141.

32. Trogdon JG, Falchook AD, Basak R, Carpenter WR, Chen RC. Total medicare costs associated with diagnosis and treatment of prostate cancer in elderly men. JAMA Oncol. 2019 Jan 1;5(1):60-66.

33. Kinsella N, Helleman J, Bruinsma S, Carlsson S, Cahill D, Brown C, et al. Active surveillance for prostate cancer: a systematic review of contemporary worldwide practices. Transl Androl Urol. 2018 Feb;7(1):83-97.

34. Kent M, Vickers AJ. A systematic literature review of life expectancy prediction tools for patients with localized prostate cancer. J Urol. 2015 Jun;193(6):1938-1942.

35. Thurtle D, Rossi SH, Berry B, Pharoah P, Gnanapragasam VJ. Models predicting survival to guide treatment decision-making in newly diagnosed primary non-metastatic prostate cancer: a systematic review. BMJ Open. 2019 Jun 22;9(6):eo29149.

36. Jayadevappa R, Chhatre S, Wong Y-N, Wittink MN, Cook R, Morales KH, et al. Comparative effectiveness of prostate cancer treatments for patient-centered outcomes: A systematic review and meta-analysis (PRISMA Compliant). Medicine. 2017 May;96(18):e6790.

37. Ricci-Cabello I, Violán C, Foguet-Boreu Q, Mounce LTA, Valderas JM. Impact of multi-morbidity on quality of healthcare and its implications for health policy, research and clinical practice. A scoping review. Eur J Gen Pract. 2015 Jul 31;21(3):192-202. 
38. Loeb S, Walter D, Curnyn C, Gold HT, Lepor H, Makarov DV. How Active is Active Surveillance? Intensity of Followup during Active Surveillance for Prostate Cancer in the United States. J Urol. 2016 Sep;196(3):721-726.

39. Butler SS, Loeb S, Cole AP, Zaslowe-Dude C, Muralidhar V, Kim DW, et al. United States trends in active surveillance or watchful waiting across patient socioeconomic status from 2010 to 2015. Prostate Cancer Prostatic Dis. 2020;23(1):179-183.

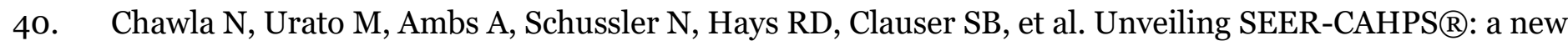
data resource for quality of care research. J Gen Intern Med. 2015 May;30(5):641-650.

41. Zaslavsky AM, Ayanian JZ, Zaborski LB. The validity of race and ethnicity in enrollment data for Medicare beneficiaries. Health Serv Res. 2012 Jun;47(3 Pt 2):1300-1321.

42. Health Resource \& Service Administration. Area Health Resources Files [Internet]. Area Health Resources Files. 2019 [cited 2020 Feb 12]. Available from: https://data.hrsa.gov/topics/healthworkforce/ahrf

43. Introduction to Boosted Trees - xgboost 1.3.0-SNAPSHOT documentation [Internet]. [cited 2020 Oct 29]. Available from: https://xgboost.readthedocs.io/en/latest/tutorials/model.html

44. Breiman L. Statistical Modeling: The Two Cultures. Stat Sci. 2001;16(3):199-231.

45. $\quad$ Lundberg SM, Lee S-I. A Unified Approach to Interpreting Model Predictions. 2017 Jan 1;

46. Loeb S, Byrne N, Makarov DV, Lepor H, Walter D. Use of Conservative Management for Low-Risk Prostate Cancer in the Veterans Affairs Integrated Health Care System From 2005-2015. JAMA. 2018 Jun 5;319(21):2231-2233.

47. DuGoff EH, Canudas-Romo V, Buttorff C, Leff B, Anderson GF. Multiple chronic conditions and life expectancy: a life table analysis. Med Care. 2014 Aug;52(8):688-694.

48. National Cancer Institute. U.S. Population Data 1969-2017 - SEER Population Data [Internet]. Surveillance, Epidemiology, and End Results (SEER) Program Populations (1969-2017) (www.seer.cancer.gov/popdata), National Cancer Institute, DCCPS, Surveillance Research Program. 2018 [cited 2019 Sep 21]. Available from: https://seer.cancer.gov/popdata/

49. Fiano RM, Merrick GS, Innes KE, Mattes MD, LeMasters TJ, Shen C, et al. Associations of multimorbidity and patient-reported experiences of care with conservative management among elderly patients with localized prostate cancer. Cancer Med. 2020 Aug;9(16):6051-6061.

50. Anderson G, Robert Wood Johnson Foundation. Chronic Care: Making the Case for Ongoing Care [Internet]. Chronic Care: Making the Case for Ongoing Care. 2010 [cited 2020 Jan 27]. Available from: https://www.rwjf.org/en/library/research/2010/o1/chronic-care.html

51. Sypes EE, de Grood C, Clement FM, Parsons Leigh J, Whalen-Browne L, Stelfox HT, et al. Understanding the public's role in reducing low-value care: a scoping review. Implement Sci. 2020 Apr 7;15(1):20.

52. Doupe P, Faghmous J, Basu S. Machine learning for health services researchers. Value Health. 2019;22(7):808-815.

53. Modi PK, Kaufman SR, Qi J, Lane BR, Cher ML, Miller DC, et al. National Trends in Active Surveillance for Prostate Cancer: Validation of Medicare Claims-based Algorithms. Urology. 2018 Oct;120:96-102.

54. Daskivich TJ, Thomas I-C, Luu M, Shelton JB, Makarov DV, Skolarus TA, et al. External Validation of the Prostate Cancer Specific Comorbidity Index: A Claims Based Tool for the Prediction of Life Expectancy in Men with Prostate Cancer. J Urol. 2019 Aug 8;202(3):518-524.

55. Goodman RA, Posner SF, Huang ES, Parekh AK, Koh HK. Defining and measuring chronic conditions: imperatives for research, policy, program, and practice. Prev Chronic Dis. 2013 Apr 25;10:E66.

56. Bice TW, Boxerman SB. A quantitative measure of continuity of care. Med Care. 1977 Apr;15(4):347349. 
57. Pinheiro LC, Reshetnyak E, Safford MM, Nanus D, Kern LM. Differences in ambulatory care fragmentation between cancer survivors and noncancer controls. Cancer. 2020 Jul 1;126(13):30943101.

58. Pollack CE, Hussey PS, Rudin RS, Fox DS, Lai J, Schneider EC. Measuring Care Continuity: A Comparison of Claims-based Methods. Med Care. 2016 May;54(5):e30-4.

59. Liu CW, Einstadter D, Cebul RD. Care fragmentation and emergency department use among complex patients with diabetes. Am J Manag Care. 2010 Jun;16(6):413-420.

60. Schwartz AL, Zaslavsky AM, Landon BE, Chernew ME, McWilliams JM. Low-Value Service Use in Provider Organizations. Health Serv Res. 2018;53(1):87-119.

61. Segal JB, Bridges JFP, Chang H-Y, Chang E, Nassery N, Weiner J, et al. Identifying possible indicators of systematic overuse of health care procedures with claims data. Med Care. 2014 Feb;52(2):157-163.

62. Reid RO, Rabideau B, Sood N. Impact of consumer-directed health plans on low-value healthcare. Am J Manag Care. 2017 Dec;23(12):741-748.

63. Mahal BA, Butler S, Franco I, Spratt DE, Rebbeck TR, D’Amico AV, et al. Use of Active Surveillance or Watchful Waiting for Low-Risk Prostate Cancer and Management Trends Across Risk Groups in the United States, 2010-2015. JAMA. 2019 Feb 19;321(7):704-706.

64. Aizer AA, Gu X, Chen M-H, Choueiri TK, Martin NE, Efstathiou JA, et al. Cost implications and complications of overtreatment of low-risk prostate cancer in the United States. J Natl Compr Canc Netw. 2015 Jan;13(1):61-68.

65. Raval AD, Madhavan S, Mattes MD, Sambamoorthi U. Types of chronic conditions combinations and initial cancer treatment among elderly Medicare beneficiaries with localised prostate cancer. Int J Clin Pract. 2016 Jul;70(7):606-618.

66. Schwartz AL, Jena AB, Zaslavsky AM, McWilliams JM. Analysis of Physician Variation in Provision of Low-Value Services. JAMA Intern Med. 2019 Jan 1;179(1):16-25.

67. Womble PR, Montie JE, Ye Z, Linsell SM, Lane BR, Miller DC, et al. Contemporary use of initial active surveillance among men in Michigan with low-risk prostate cancer. Eur Urol. 2015 Jan;67(1):44-50.

68. Löppenberg B, Friedlander DF, Krasnova A, Tam A, Leow JJ, Nguyen PL, et al. Variation in the use of active surveillance for low-risk prostate cancer. Cancer. 2018 Jan 1;124(1):55-64.

69. Boehm K, Dell'Oglio P, Tian Z, Capitanio U, Chun FKH, Tilki D, et al. Comorbidity and age cannot explain variation in life expectancy associated with treatment of non-metastatic prostate cancer. World J Urol. 2017 Jul;35(7):1031-1036.

70. Chen S, Bergman D, Miller K, Kavanagh A, Frownfelter J, Showalter J. Using applied machine learning to predict healthcare utilization based on socioeconomic determinants of care. Am J Manag Care. 2020;26(1):26-31.

71. Al Hussein Al Awamlh B, Ma X, Christos P, Hu JC, Shoag JE. Active Surveillance for Black Men with Low-Risk Prostate Cancer in the United States. N Engl J Med. 2019 Dec 26;381(26):2581-2582.

72. Parikh RB, Robinson KW, Chhatre S, Medvedeva E, Cashy JP, Veera S, et al. Comparison by Race of Conservative Management for Low-Risk and Intermediate-Risk Prostate Cancers in Veterans From 2004 to 2018. JAMA Netw Open. 2020 Sep 1;3(9):e2018318.

73. Center for Disease Control and Prevention. Health in All Policies | AD for Policy and Strategy | CDC [Internet]. 2015 [cited 2020 Jun 8]. Available from: https://www.cdc.gov/policy/hiap/index.html

74. Sequist TD, Schneider EC, Anastario M, Odigie EG, Marshall R, Rogers WH, et al. Quality monitoring of physicians: linking patients' experiences of care to clinical quality and outcomes. J Gen Intern Med. 2008 Nov;23(11):1784-1790.

75. Garg R, Shen C, Sambamoorthi N, Kelly K, Sambamoorthi U. Type of Multimorbidity and PatientDoctor Communication and Trust among Elderly Medicare Beneficiaries. Int J Family Med. 2016 Oct 5;2016:8747891. 
76. Johnston MC, Crilly M, Black C, Prescott GJ, Mercer SW. Defining and measuring multimorbidity: a systematic review of systematic reviews. Eur J Public Health. 2019 Feb 1;29(1):182-189.

77. Daskivich TJ, Fan K-H, Koyama T, Albertsen PC, Goodman M, Hamilton AS, et al. Effect of age, tumor risk, and comorbidity on competing risks for survival in a U.S. population-based cohort of men with prostate cancer. Ann Intern Med. 2013 May 21;158(10):709-717.

78. Klabunde CN, Potosky AL, Legler JM, Warren JL. Development of a comorbidity index using physician claims data. J Clin Epidemiol. 200o Dec;53(12):1258-1267.

79. Klinkman MS. Competing demands in psychosocial care. A model for the identification and treatment of depressive disorders in primary care. Gen Hosp Psychiatry. 1997 Mar;19(2):98-111.

80. National Cancer Institute. Case-Mix Adjustment Guidance [Internet]. Guidance on Standard Covariate Adjustment for SEER-CAHPS Analyses. 2019 [cited 2020 Feb 13]. Available from:

https://healthcaredelivery.cancer.gov/seer-cahps/researchers/adjustment_guidance.html

81. Li C. Little's test of missing completely at random. The Stata Journal. 2013 Dec;13(4):795-809.

82. Auffenberg GB, Lane BR, Linsell S, Cher ML, Miller DC. Practice- vs Physician-Level Variation in Use of Active Surveillance for Men With Low-Risk Prostate Cancer: Implications for Collaborative Quality Improvement. JAMA Surg. 2017 Oct 1;152(10):978-980.

83. Lang MF, Tyson MD, Alvarez JR, Koyama T, Hoffman KE, Resnick MJ, et al. The Influence of Psychosocial Constructs on the Adherence to Active Surveillance for Localized Prostate Cancer in a Prospective, Population-based Cohort. Urology. 2017 Feb 9;103:173-178.

84. Tyson MD, Graves AJ, O’Neil B, Barocas DA, Chang SS, Penson DF, et al. Urologist-Level Correlation in the Use of Observation for Low- and High-Risk Prostate Cancer. JAMA Surg. 2017 Jan 1;152(1):27-34.

85. D'Amico TA, Bandini LAM, Balch A, Benson AB, Edge SB, Fitzgerald CL, et al. Quality Measurement in Cancer Care: A Review and Endorsement of High-Impact Measures and Concepts. J Natl Compr Canc Netw. 2020;18(3):250-259.

86. Bekelman JE, Rumble RB, Chen RC, Pisansky TM, Finelli A, Feifer A, et al. Clinically localized prostate cancer: ASCO clinical practice guideline endorsement of an american urological association/american society for radiation oncology/society of urologic oncology guideline. J Clin Oncol. 2018 Nov 10;36(32):3251-3258.

87. Volk RJ, McFall SL, Cantor SB, Byrd TL, Le Y-CL, Kuban DA, et al. It“s not like you just had a heart attack": decision-making about active surveillance by men with localized prostate cancer.

Psychooncology. 2014 Apr;23(4):467-472.

88. Le Y-CL, McFall SL, Byrd TL, Volk RJ, Cantor SB, Kuban DA, et al. Is "Active Surveillance” an Acceptable Alternative?: A Qualitative Study of Couples’ Decision Making about Early-Stage, Localized Prostate Cancer. Narrat Inq Bioeth. 2016;6(1):51-61.

89. Latini DM, Hart SL, Knight SJ, Cowan JE, Ross PL, Duchane J, et al. The relationship between anxiety and time to treatment for patients with prostate cancer on surveillance. J Urol. 2007 Sep;178(3 Pt 1):826-831.

90. Orom H, Nelson CJ, Underwood W, Homish DL, Kapoor DA. Factors associated with emotional distress in newly diagnosed prostate cancer patients. Psychooncology. 2015 Nov;24(11):1416-1422.

91. Goh AC, Kowalkowski MA, Bailey DE, Kazer MW, Knight SJ, Latini DM. Perception of cancer and inconsistency in medical information are associated with decisional conflict: a pilot study of men with prostate cancer who undergo active surveillance. BJU Int. 2012 Jul;110(2 Pt 2):E50-6.

92. Filson CP, Schroeck FR, Ye Z, Wei JT, Hollenbeck BK, Miller DC. Variation in use of active surveillance among men undergoing expectant treatment for early stage prostate cancer. J Urol. 2014 Jul;192(1):7580.

93. Daniels N. Equity of access to health care: some conceptual and ethical issues. Milbank Mem Fund Q Health Soc. 1982;60(1):51-81. 
94. Kinsella N, Helleman J, Bruinsma S, Carlsson S, Cahill D, Brown C, et al. Active surveillance for prostate cancer: a systematic review of contemporary worldwide practices. Translational Andrology and Urology; Vol 7, No 1 (February 2018): Translational Andrology and Urology (Prostate Cancer Screening and Active Surveillance in the Western World). 2018;

95. Ehdaie B, Assel M, Benfante N, Malhotra D, Vickers A. A Systematic Approach to Discussing Active Surveillance with Patients with Low-risk Prostate Cancer. Eur Urol. 2017 Jan 24;71(6):866-871.

96. Xu J, Neale AV, Dailey RK, Eggly S, Schwartz KL. Patient perspective on watchful waiting/active surveillance for localized prostate cancer. J Am Board Fam Med. 2012 Dec;25(6):763-770.

97. Ennis RD, Parikh AB, Sanderson M, Liu M, Isola L. Interpreting Oncology Care Model Data to Drive Value-Based Care: A Prostate Cancer Analysis. J Oncol Pract. 2019 Mar;15(3):e238-e246.

98. Osawa I, Goto T, Yamamoto Y, Tsugawa Y. Machine-learning-based prediction models for high-need high-cost patients using nationwide clinical and claims data. npj Digital Med. 2020 Nov 11;3(1):148.

99. $\mathrm{Hu} \mathrm{L}$, Li L, Ji J, Sanderson M. Identifying and understanding determinants of high healthcare costs for breast cancer: a quantile regression machine learning approach. BMC Health Serv Res. 2020 Nov 23;20(1):1066.

100. US Department of Labor. Bureau of Labor Statistics [Internet]. 2015 [cited 2020 Feb 28]. Available from: http://www.bls.gov/news.release/archives/cpi_06182015.pdf

101. Volpp KG, Loewenstein G, Asch DA. Choosing wisely: low-value services, utilization, and patient cost sharing. JAMA. 2012 Oct 24;308(16):1635-1636.

102. Modi PK, Kaufman SR, Herrel LA, Dupree JM, Luckenbaugh AN, Skolarus TA, et al. Practice-Level Adoption of Conservative Management for Prostate Cancer. J Oncol Pract. 2019 Sep 11;JOP1900o88.

103. Xu J, Janisse J, Ruterbusch JJ, Ager J, Liu J, Holmes-Rovner M, et al. Patients' survival expectations with and without their chosen treatment for prostate cancer. Ann Fam Med. 2016;14(3):208-214.

104. Bill-Axelson A, Holmberg L, Garmo H, Rider JR, Taari K, Busch C, et al. Radical prostatectomy or watchful waiting in early prostate cancer. N Engl J Med. 2014 Mar 6;370(10):932-942.

105. Wilt TJ, Brawer MK, Jones KM, Barry MJ, Aronson WJ, Fox S, et al. Radical prostatectomy versus observation for localized prostate cancer. N Engl J Med. 2012 Jul 19;367(3):203-213.

106. Hamdy FC, Donovan JL, Lane JA, Mason M, Metcalfe C, Holding P, et al. 10-Year Outcomes after Monitoring, Surgery, or Radiotherapy for Localized Prostate Cancer. N Engl J Med. 2016 Oct 13;375(15):1415-1424.

107. Boyd CM, Darer J, Boult C, Fried LP, Boult L, Wu AW. Clinical practice guidelines and quality of care for older patients with multiple comorbid diseases: implications for pay for performance. JAMA. 2005 Aug 10;294(6):716-724.

108. Dawes M. Co-morbidity: we need a guideline for each patient not a guideline for each disease. Fam Pract. 2010 Feb;27(1):1-2.

109. Centers for Medicare and Medicaid Services. Oncology Care Model | CMS Innovation Center [Internet]. Oncology Care Model | CMS Innovation Center. 2020 [cited 2020 Jun 8]. Available from: https://innovation.cms.gov/innovation-models/oncology-care 


\section{Appendices}

Supplemental Figure 7.o Aim 1 Inclusion \& Exclusion Criteria

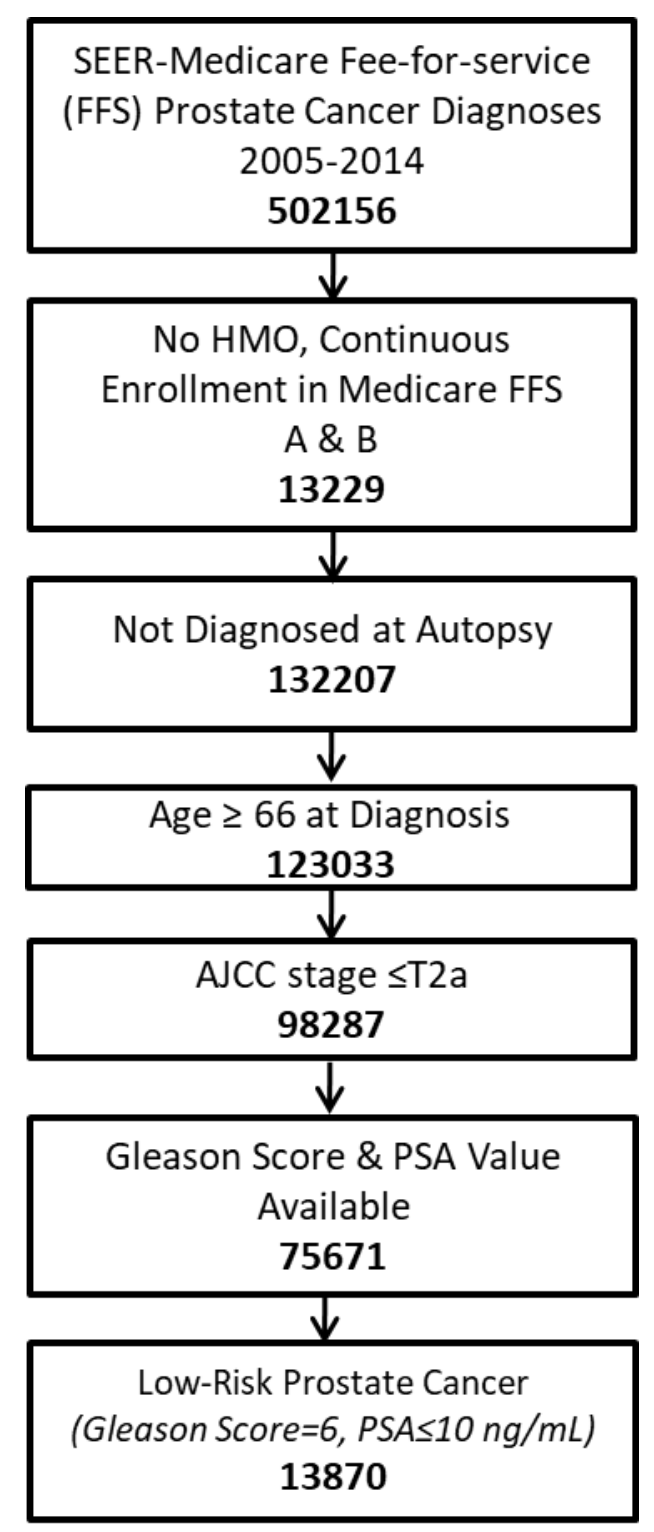




\section{Supplemental Figure 7.1 Feature Importance of Treatment Prediction among Fee-for-Service Medicare Beneficiaries with Incident Low-risk Prostate Cancer using SEER Cancer Registry, 2009-2014 (n=13870)}

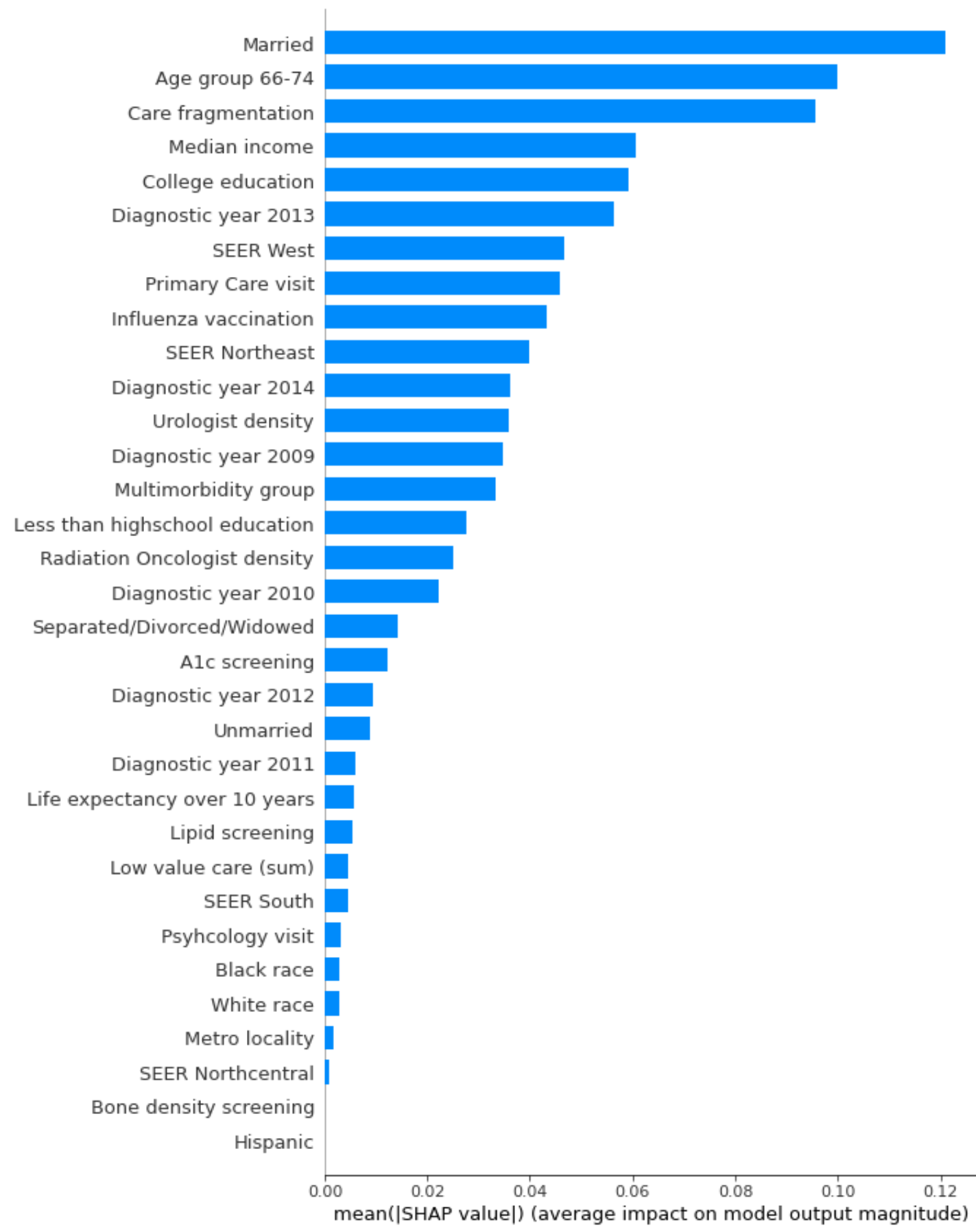

Mean SHAP values in descending order of log-odds. Based on 13870 older (age $\geq 66$ years) Fee-for-Service Medicare beneficiaries, with continuous enrollment in Medicare Part A \& Part B, diagnosed with incident lowrisk prostate cancer (Gleason 6, PSA <10, Stage sT2a) between 2009 and 2014.

Age group included 66-74 and 75 and over categories.

Care fragmentation = Bice-Boxerman continuity of care index to calculate care fragmentation during the 12 month baseline period (See Methods).

SEER = Surveillance, Epidemiology, and End Results cancer Registry. 
Appendix 7.2 Unadjusted and Adjusted Odds Ratios and 95\% Confidence Intervals (CI) of PCCI, Mental Health, Timeliness of Care, Low-risk Prostate Cancer, and Education on Conservative Management Use in among Fee-for-Service Medicare Beneficiaries with Incident Localized Prostate Cancer using Linked SEER Cancer Registry with MCAHPS, 2002-2013 ( $\mathrm{N}=496)$.

\begin{tabular}{|c|c|c|c|c|c|c|}
\hline \multirow[b]{2}{*}{ Prostate Cancer Comorbidity Index } & \multicolumn{2}{|c|}{ UOR [95\% CI] } & \multirow[t]{2}{*}{ p-value } & \multicolumn{2}{|c|}{$\operatorname{AOR}[95 \% \mathrm{CI}]$} & \multirow[t]{2}{*}{ p-value } \\
\hline & & & & & & \\
\hline 5 to 10 years life expectancy & 0.58 & [0.38-0.90] & 0.014 & 0.48 & {$[0.29-0.78]$} & 0.003 \\
\hline$<5$ years life expectancy & 0.58 & {$[0.33-1.03]$} & 0.062 & 0.42 & {$[0.21-0.83]$} & 0.012 \\
\hline \multicolumn{7}{|l|}{$>10$ years life expectancy (Ref.) } \\
\hline Getting Care Quickly & 1.15 & {$[1.05-1.27]$} & 0.003 & 1.20 & {$[1.09-1.34]$} & 0.001 \\
\hline \multicolumn{7}{|l|}{ Low-risk prostate cancer } \\
\hline Yes & 1.41 & {$[0.95-2.08]$} & 0.088 & 1.65 & {$[1.07-2.58]$} & 0.024 \\
\hline \multicolumn{7}{|l|}{ No (Ref.) } \\
\hline \multicolumn{7}{|l|}{ Mental Health } \\
\hline Fair/Poor & 2.97 & {$[1.50-5.90]$} & 0.002 & 5.54 & {$[2.33-13.2]$} & 0.001 \\
\hline \multicolumn{7}{|l|}{ Excellent/Very Good (Ref.) } \\
\hline \multicolumn{7}{|l|}{ Education } \\
\hline College or more & 2.49 & {$[1.30-4.78]$} & 0.006 & 3.28 & {$[1.52-7.10]$} & 0.002 \\
\hline High-school graduate & 2.41 & {$[1.18-4.92]$} & 0.015 & 3.57 & {$[1.60-7.96]$} & 0.002 \\
\hline No high-school grad. (Ref.) & & & & & & \\
\hline
\end{tabular}


Appendix 7.3 Unadjusted and Adjusted Odds Ratios and 95\% Confidence Intervals of PCCI, Multimorbidity, Mental Health, Timeliness of Care, Low-risk Prostate Cancer, and Education on Conservative Management Use up to 24 Months after Incident Prostate Cancer among Fee-for-Service Medicare Beneficiaries using Linked SEER Cancer Registry with MCAHPS, 2002-2013 ( $\mathrm{N}=496)$.

\begin{tabular}{|c|c|c|c|c|c|c|}
\hline & \multicolumn{3}{|c|}{ UOR [95\% CI] } & \multicolumn{2}{|c|}{ AOR $[95 \% \mathrm{CI}]$} & p-value \\
\hline \multicolumn{7}{|c|}{ Multimorbidity Model } \\
\hline \multicolumn{7}{|l|}{ Multimorbidity } \\
\hline Yes & 0.52 & {$[0.35-0.77]$} & 0.001 & 0.42 & {$[0.27-0.65]$} & $<0.001$ \\
\hline \multicolumn{7}{|l|}{ No (Ref.) } \\
\hline Getting Care Quickly & 1.13 & {$[1.02-1.24]$} & 0.014 & 1.21 & {$[1.09-1.35]$} & $<0.001$ \\
\hline \multicolumn{7}{|l|}{ Low-risk prostate cancer } \\
\hline Yes & 1.46 & {$[0.97-2.18]$} & 0.068 & 1.76 & {$[1.14-2.72]$} & 0.01 \\
\hline \multicolumn{7}{|l|}{ No (Ref.) } \\
\hline \multicolumn{7}{|l|}{ Mental Health } \\
\hline Fair/Poor & 3.44 & {$[1.73-6.81]$} & $<0.001$ & 4.32 & {$[1.86-10.1]$} & $<0.001$ \\
\hline \multicolumn{7}{|l|}{ Excellent/Very Good (Ref.) } \\
\hline \multicolumn{7}{|l|}{ Education } \\
\hline College or more & 2.76 & {$[1.35-5.65]$} & 0.006 & 3.21 & {$[1.50-6.89]$} & 0.003 \\
\hline High-school graduate & 3.06 & {$[1.42-6.63]$} & 0.004 & 3.53 & {$[1.59-7.83]$} & 0.002 \\
\hline No high-school graduation (Ref.) & & & & & & \\
\hline \multicolumn{7}{|c|}{ PCCI Model } \\
\hline \multicolumn{7}{|l|}{ Prostate Cancer Comorbidity Index } \\
\hline 5 to 10 years life expectancy & 0.52 & {$[0.33-0.82]$} & 0.005 & 0.39 & {$[0.30-0.70]$} & $<0.001$ \\
\hline$<5$ years life expectancy & 0.56 & {$[0.31-1.01]$} & 0.056 & 0.34 & {$[0.16-0.73]$} & 0.005 \\
\hline \multicolumn{7}{|l|}{$>10$ years life expectancy (Ref.) } \\
\hline Getting Care Quickly & & & & 1.20 & {$[1.08-1.34]$} & 0.001 \\
\hline Low-risk prostate cancer & & & & 1.76 & {$[1.11-2.79]$} & 0.017 \\
\hline \multicolumn{7}{|l|}{ Yes } \\
\hline \multicolumn{7}{|l|}{ No (Ref.) } \\
\hline \multicolumn{7}{|l|}{ Mental Health } \\
\hline Fair/Poor & & & & 7.59 & {$[3.07-18.8]$} & $<0.001$ \\
\hline \multicolumn{7}{|l|}{ Excellent/Very Good (Ref.) } \\
\hline \multicolumn{7}{|l|}{ Education } \\
\hline High-school graduate & & & & 0.26 & {$[0.11-0.60]$} & 0.002 \\
\hline No high-school grad. (Ref.) & & & & & & \\
\hline
\end{tabular}

Note: Based on 496 older (age $\geq 66$ years) Fee-for-Service Medicare beneficiaries, with continuous enrollment in Medicare part A \& Part B, diagnosed with incident localized prostate cancer between 2003 and 2013. Adjusted for age, race, marital status, income, education, health status, urologist density, radiation oncologist density, SEER region, geography, diagnostic year, and low-risk prostate cancer.

$\mathrm{UOR}=$ Unadjusted Odds Ratio, AOR= Adjusted Odds Ratio, N.S.= Not significant, $\mathrm{CI}=$ Confidence interval, Ref= Reference group, SEER = Surveillance, Epidemiology and End Results cancer Registry, MCAHPS= Medicare Consumer Assessment of Healthcare Providers and System surveys, CM= Conservative management, PCCI= Prostate Cancer Comorbidity Index. Statistically significant results displayed. 


\begin{tabular}{|c|c|}
\hline \multicolumn{2}{|c|}{$\begin{array}{l}\text { Appendix 7.4 Prostate cancer treatment codes used within } 12 \text { months of prostate cancer diagnosis to Identify } \\
\text { Conservative Management Use }\end{array}$} \\
\hline Radical prostatectomy & $\begin{array}{l}55840,55842,55845,55866,55810,55812,55815 \text { and } 60.62,17.42,60.5,60.4 \text {, and } \\
60.3(40.3,40.53 \text {, and } 40.59 \text { for lymph node dissection) }\end{array}$ \\
\hline Brachytherapy & $\begin{array}{l}\text { 76873, 55859, 76965, 55860, 55875, 55876, 76873, 76965, 77326, 77327, 77328, } \\
\text { 77761, 77762, 77763, 77799, 77776, 77777, 77778, and 60.99, 92.27, 92.28, and } \\
\text { 92.29, 77781, 77782, 77783, 77784, 77785, 77786, 77787, 77789, 77790, Q3001, } \\
\text { A9527, C1715, C1716, C1717, C1719, C1728, C2616, C2634, C2635, C2636, C2637, } \\
\text { C2638, C2639, C2640, C2641, C2642, C2643, C2698, C2699, C9725 }\end{array}$ \\
\hline $\begin{array}{l}\text { External Beam } \\
\text { Radiotherapy }\end{array}$ & $\begin{array}{l}\text { 77305, 77310, 77315, 77321, 77371,77372, 77373, 77402, 77403, 77404, 77406, } \\
\text { 77407, 77408, 77409, 77411, 77412, 77413, 77414, 77416, 77422, 77423, 92.24, 92.26, } \\
\text { 77301, 77418, 0073T, 77380, 77381, 77520, 77522, 77523, and 77525, 77301,77338, } \\
\text { 77761, 77762, 77763, 77789, 77427 }\end{array}$ \\
\hline Cryotherapy & $50250,50593,55873,55873,60.62$, C2618, Go160, Go161 \\
\hline $\begin{array}{c}\text { Androgen Deprivation } \\
\text { Therapy }\end{array}$ & $\begin{array}{l}\text { J1050, J1051 (Progesterone),J1950 J9217 J9218 J9219, C9430 (Lupron), J9165 } \\
\text { (DES), J9202 (Zoladex), J3315 (Trelstar), J9225 CPT 11981(Vantas), all injections } \\
\text { ICD-99.24, So175 (Flutamide), J8999 (Bicalutamide/Flutamide/Nilutamide) } \\
\text { So165, Jo128, C9216, (Abarelix), J9155 (Degarelix), S9560 (any hormone/adt), } \\
\text { G0356 (any ADT ) }\end{array}$ \\
\hline $\begin{array}{l}\text { Conservative } \\
\text { management }\end{array}$ & nent within one year of prostate cancer diagnosis \\
\hline
\end{tabular}


Appendix 7.5 Patient Characteristics by Conservative Management among Fee-for-Service Medicare Beneficiaries with Incident Localized Prostate Cancer using Linked SEER Cancer Registry, 2005-2014 $(\mathrm{n}=75671)$

\begin{tabular}{|c|c|c|c|c|c|c|c|}
\hline & & \multicolumn{2}{|l|}{$\mathbf{C M}$} & \multicolumn{2}{|l|}{ No CM } & \multirow[b]{2}{*}{$\mathbf{X}^{2}$} & \multirow[b]{2}{*}{ p-value } \\
\hline & & $\mathbf{N}$ & $\%$ & $\mathbf{N}$ & $\%$ & & \\
\hline ALL & & 13662 & 18.1 & 62009 & 82.0 & & \\
\hline \multicolumn{2}{|c|}{ Age in Years } & & & & & 107.6 & $<0.001$ \\
\hline & $66-74$ & 8424 & 17.0 & 41125 & 83.0 & & \\
\hline & $75^{+}$ & 5238 & 20.1 & 20884 & 79.9 & & \\
\hline \multicolumn{2}{|l|}{ Race } & & & & & 145.2 & $<0.001$ \\
\hline & White & 10741 & 17.4 & 50813 & 82.6 & & \\
\hline & Black & 1941 & 22.5 & 6671 & 77.5 & & \\
\hline & Hispanic & 188 & 14.7 & 1091 & $85 \cdot 3$ & & \\
\hline & Other & 750 & 18.6 & 3289 & 81.4 & & \\
\hline \multicolumn{2}{|c|}{ Marital Status } & & & & & $813 \cdot 3$ & $<0.001$ \\
\hline & Married & 8190 & 15.6 & 44303 & 84.4 & & \\
\hline & Unmarried & 997 & 19.4 & 4155 & 80.6 & & \\
\hline & Separated/Divorced/Widowed & 2204 & 23.2 & 7293 & 76.8 & & \\
\hline & Unknown & 2271 & 26.6 & 6258 & 73.4 & & \\
\hline \multicolumn{2}{|c|}{ Income quintiles } & & & & & 35.5 & $<0.001$ \\
\hline & First & 2772 & 18.9 & 11912 & 81.1 & & \\
\hline & Second & 2620 & 17.8 & 12133 & 82.2 & & \\
\hline & Third & 2696 & 18.3 & 12073 & 81.7 & & \\
\hline & Four & 2558 & 17.4 & 12160 & 82.6 & & \\
\hline & Fifth & 2642 & 17.5 & 12426 & 82.5 & & \\
\hline \multicolumn{2}{|c|}{ Education quintiles } & & & & & 102.0 & $<0.001$ \\
\hline & First & 2604 & 17.7 & 12125 & 82.3 & & \\
\hline & Second & 2461 & 16.6 & 12349 & 83.4 & & \\
\hline & Third & 2496 & 16.8 & 12356 & 83.2 & & \\
\hline & Four & 2758 & 18.6 & 12055 & 81.4 & & \\
\hline & Fifth & 2984 & 20.1 & 11874 & 79.9 & & \\
\hline \multicolumn{2}{|c|}{ Urologist density quintiles } & & & & & 33.0 & $<0.001$ \\
\hline & First & 2509 & 16.6 & 12562 & 83.4 & & \\
\hline & Second & 2820 & 18.3 & 12559 & 81.7 & & \\
\hline & Third & 2689 & 18.6 & 11762 & 81.4 & & \\
\hline & Four & 2817 & 18.1 & 12738 & 81.9 & & \\
\hline & Fifth & 2798 & 18.5 & 12313 & 81.5 & & \\
\hline
\end{tabular}




\begin{tabular}{|c|c|c|c|c|c|c|}
\hline \multicolumn{7}{|l|}{ Continued Appendix 7.5} \\
\hline Radiation oncologist quintiles & & & & & 58.18 & $<0.001$ \\
\hline First & 2631 & 17.1 & 12783 & 82.9 & & \\
\hline Second & 2421 & 16.6 & 12183 & 83.4 & & \\
\hline Third & 2756 & 18.6 & 12054 & 81.4 & & \\
\hline Four & 2960 & 18.9 & 12667 & 81.1 & & \\
\hline Fifth & 2865 & 19.0 & 12247 & 81.0 & & \\
\hline Low-risk prostate cancer & & & & & 2600 & $<0.001$ \\
\hline GS $>6$ and/or PSA $\geq 10 n g / m L$ & 6245 & 12.8 & 42513 & 87.2 & & \\
\hline GS 6 and PSA $<10 n g / m L$ & 7417 & 27.6 & 19496 & 72.4 & & \\
\hline Multimorbidity & & & & & 752.3 & $<0.001$ \\
\hline o-2 Chronic conditions & 7465 & 22.4 & 25901 & 77.6 & & \\
\hline$>2$ Chronic conditions & 6197 & 14.6 & 36108 & 85.4 & & \\
\hline Prostate Cancer Comorbidity Index & & & & & 67.6 & $<0.001$ \\
\hline 10 or more years life expectancy & 10780 & 18.6 & 47103 & 81.4 & & \\
\hline 5-10 years life expectancy & 1927 & 15.5 & 10503 & 84.5 & & \\
\hline 5 or less years life expectancy & 955 & 17.8 & 4403 & 82.2 & & \\
\hline Low-value care & & & & & 206.7 & $<0.001$ \\
\hline Low-value care & 3070 & 15.2 & 17140 & 84.8 & & \\
\hline No low-value care & 10592 & 19.1 & 44869 & 80.9 & & \\
\hline Preventative Arc test & & & & & 38.8 & $<0.001$ \\
\hline A1c test & 1590 & 15.8 & 8456 & 84.2 & & \\
\hline No a1c test & 12072 & 18.4 & 53553 & 81.6 & & \\
\hline Preventative flu & & & & & 422.4 & $<0.001$ \\
\hline Influenza vaccination & 4420 & 14.5 & 25966 & 85.5 & & \\
\hline No influenza vaccination & 9242 & 20.4 & 36043 & 79.6 & & \\
\hline Preventative lipid screen & & & & & & $<0.001$ \\
\hline Lipid test & 3161 & 15.2 & 17629 & 84.8 & & \\
\hline No lipid test & 10501 & 19.1 & 44380 & 80.9 & & \\
\hline Metro & & & & & 14.2 & $<0.001$ \\
\hline Metro county & 11586 & 18.2 & 52052 & 81.8 & & \\
\hline Non-metro county & 2047 & 17.2 & 9882 & 82.8 & & \\
\hline SEER Region & & & & & 458.1 & $<0.001$ \\
\hline Northeast & 2137 & 13.8 & 13382 & 86.2 & & \\
\hline South & 3296 & 17.0 & 16067 & 83.0 & & \\
\hline North Central & 1370 & 16.0 & 7193 & 84.0 & & \\
\hline West & 6859 & 21.3 & 25367 & 78.7 & & \\
\hline Year of diagnosis & & & & & 198.4 & $<0.001$ \\
\hline$<2009$ & 6737 & 20.3 & 26481 & 79.7 & & \\
\hline
\end{tabular}


Appendix 7.6 Low-value Care by Conservative Management Use among Fee-for-Service Medicare Beneficiaries with Incident Localized Prostate Cancer using Linked SEER Cancer Registry, 2005-2014 $(\mathrm{n}=75671)$

\begin{tabular}{|c|c|c|c|c|c|c|c|c|}
\hline & $\begin{array}{l}\mathrm{LVC} \\
(\mathrm{n}=19\end{array}$ & 071) & $\begin{array}{l}\mathrm{CM} \\
(\mathrm{n}=136\end{array}$ & & No CM & $(n=62009)$ & & \\
\hline & $\mathbf{N}$ & LVC\% & $\mathbf{N}$ & CM\% & $\mathbf{N}$ & No CM \% & $\mathbf{X}^{2}$ & p-value \\
\hline Preoperative chest radiography & 2010 & 2.66 & & & & & 23.738 & $<0.001^{*}$ \\
\hline Yes & & & 280 & 13.9 & 1730 & 86.1 & & \\
\hline No & & & 13382 & 18.2 & 60279 & 81.8 & & \\
\hline Preoperative echocardiography & 1008 & $1.33 \%$ & & & & & 11.982 & $<0.001^{*}$ \\
\hline Yes & & & 140 & 13.9 & 868 & 86.1 & & \\
\hline No & & & 13522 & 18.1 & 61141 & 81.9 & & \\
\hline $\begin{array}{l}\text { Arthroscopic surgery for knee } \\
\text { osteoarthritis }\end{array}$ & 33 & 0.04 & & & & & 1.793 & 0.181 \\
\hline Yes & & & $* *$ & $* *$ & ** & & & \\
\hline No & & & *** & $* *$ & ** & & & \\
\hline $\begin{array}{l}\text { Hypercoagulability testing for } \\
\text { patients with DVT }\end{array}$ & 78 & 0.10 & & & & & 0.102 & 0.750 \\
\hline Yes & & & 13 & 16.7 & 65 & 83.3 & & \\
\hline No & & & 13649 & 18.1 & 61944 & 81.9 & & \\
\hline $\begin{array}{l}\text { Stress echocardiography for CAD or } \\
\text { risk assessment }\end{array}$ & 1916 & 2.53 & & & & & 12.144 & $<0.001^{*}$ \\
\hline Yes & & & 288 & 15.0 & 1628 & 85.0 & & \\
\hline No & & & 13374 & 18.1 & 60381 & 81.9 & & \\
\hline Laminectomy or spinal fusion & 348 & 0.46 & & & & & 1.196 & 0.274 \\
\hline Yes & & & 55 & 15.8 & 293 & 84.2 & & \\
\hline No & & & 13607 & 18.1 & 61716 & 81.9 & & \\
\hline $\begin{array}{l}\text { Fiberoptic laryngoscopy for } \\
\text { sinusitis }\end{array}$ & 636 & 0.84 & & & & & 4.649 & $0.031^{*}$ \\
\hline Yes & & & 94 & 14.8 & 542 & 85.2 & & \\
\hline No & & & 13568 & 18.1 & 61467 & 81.9 & & \\
\hline $\begin{array}{l}\text { Routine monitoring of digoxin in } \\
\text { CHF patients }\end{array}$ & 613 & 0.81 & & & & & 2.731 & 0.098 \\
\hline Yes & & & 95 & $15 \cdot 5$ & 518 & 84.5 & & \\
\hline No & & & 13567 & 18.1 & 61491 & 81.9 & & \\
\hline $\begin{array}{l}\text { EEG or imaging monitoring in } \\
\text { patients with syncope }\end{array}$ & 1388 & 1.83 & & & & & 0.064 & 0.800 \\
\hline Yes & & & 247 & 17.8 & 1141 & 82.2 & & \\
\hline No & & & 13415 & 18.1 & 60868 & 81.9 & & \\
\hline $\begin{array}{l}\text { Serological tests for helicobacter } \\
\text { pylori }\end{array}$ & 732 & 0.97 & & & & & $5 \cdot 442$ & $0.020^{*}$ \\
\hline Yes & & & 108 & 14.8 & 624 & 85.2 & & \\
\hline No & & & 13554 & 18.1 & 61385 & 81.9 & & \\
\hline
\end{tabular}




\begin{tabular}{|c|c|c|c|c|c|c|c|c|}
\hline $\begin{array}{l}\text { MRI in individuals with traumatic } \\
\text { brain injury }\end{array}$ & 16 & 0.02 & & & & & 0.005 & 0.942 \\
\hline Yes & & & $* *$ & $* *$ & ** & *** & & \\
\hline No & & & $* *$ & $* *$ & *** & *** & & \\
\hline Traction for low back pain & 5759 & 7.61 & & & & & 5.850 & $0.016^{*}$ \\
\hline Yes & & & 510 & 16.4 & 2596 & 83.6 & & \\
\hline No & & & 13152 & 18.1 & 59413 & 81.9 & & \\
\hline $\begin{array}{l}\text { Screening for asymptomatic carotid } \\
\text { artery stenosis }\end{array}$ & 7367 & 9.74 & & & & & 65.614 & $<0.001^{*}$ \\
\hline Yes & & & 1076 & 14.6 & 6291 & 85.4 & & \\
\hline No & & & 12586 & 18.4 & 55718 & 81.6 & & \\
\hline $\begin{array}{l}\text { Immunoglobulin (IgG, IgE) tests } \\
\text { for evaluation of allergy }\end{array}$ & 269 & 0.36 & & & & & 1.444 & 0.230 \\
\hline Yes & & & 41 & 15.2 & 228 & 84.8 & & \\
\hline No & & & 13621 & 18.1 & 61781 & 81.9 & & \\
\hline $\begin{array}{l}\text { MRI Lumbar Spine for Low Back } \\
\text { Pain }\end{array}$ & 5759 & 7.61 & & & & & 25.889 & $<0.001^{*}$ \\
\hline Yes & & & 897 & 15.6 & 4862 & 84.4 & & \\
\hline No & & & 12765 & 18.3 & 57147 & 81.7 & & \\
\hline IVC filters to prevent PE & 41 & 0.05 & & & & & 0.059 & 0.808 \\
\hline Yes & & & ** & ** & *** & *** & & \\
\hline No & & & $* *$ & $* *$ & ** & ** & & \\
\hline Renal angioplasty or stent & 43 & 0.06 & & & & & 1.201 & 0.273 \\
\hline Yes & & & ** & $* *$ & ** & ** & & \\
\hline No & & & ** & *** & *** & *** & & \\
\hline Vitamin D screening & 462 & 0.61 & & & & & 2.648 & 0.104 \\
\hline Yes & & & 70 & 15.2 & 392 & 84.8 & & \\
\hline No & & & 13592 & 18.1 & 61617 & 81.9 & & \\
\hline $\begin{array}{l}\text { Head imaging for uncomplicated } \\
\text { headache }\end{array}$ & 332 & 0.44 & & & & & 0.018 & 0.893 \\
\hline Yes & & & 59 & 17.8 & 273 & 82.2 & & \\
\hline No & & & 13603 & 18.1 & 61736 & 81.9 & & \\
\hline Imaging for plantar fasciitis & 37 & 0.05 & & & & & 0.516 & 0.473 \\
\hline Yes & & & $* *$ & $* *$ & ** & *** & & \\
\hline No & & & $* *$ & $* *$ & $* *$ & ** & & \\
\hline
\end{tabular}


Supplemental Figure 7.7 Aim 3 Inclusion \& Exclusion Criteria

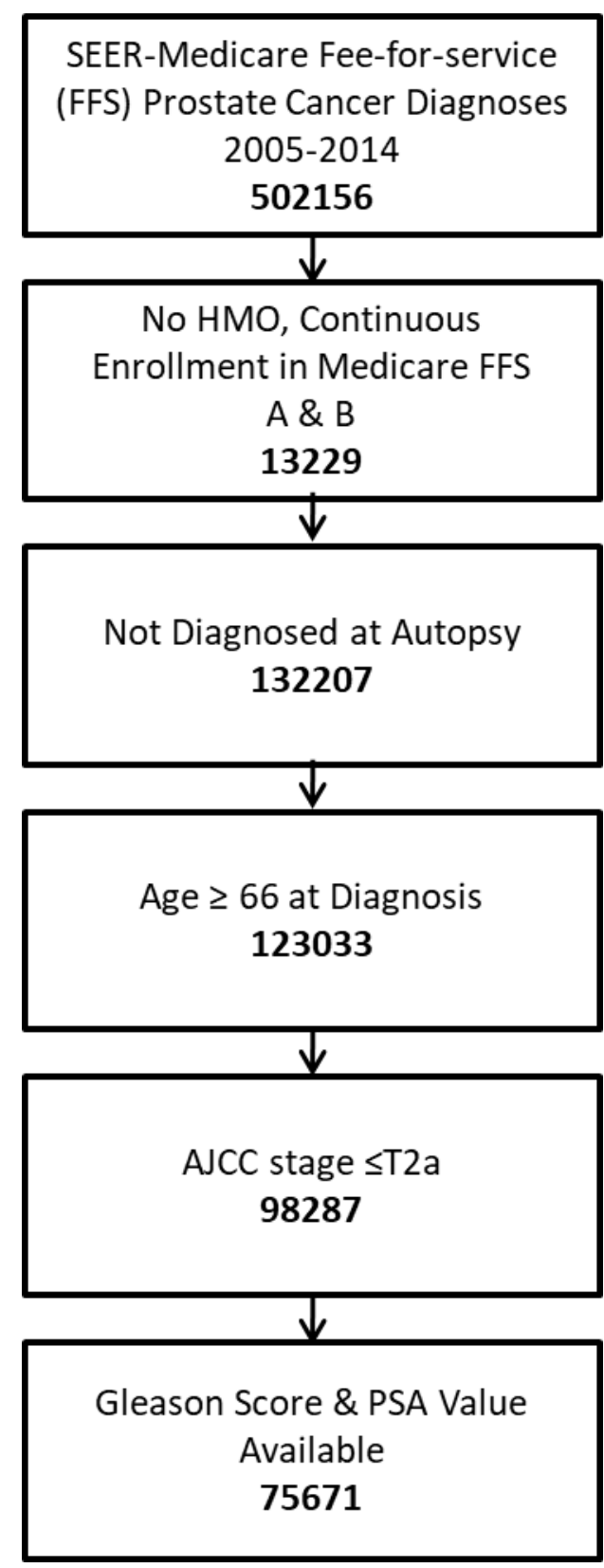




\section{Appendix 7.8 Aim 1 python codes}

import pandas as pd

import numpy as np

feature_names for SHAP analyses below

feature_names = [ "Married", "Age group 66-74", "Median income", "Care fragmentation", "Less than

highschool education", "College education", "SEER Northeast", "Primary Care visit", "Urologist density", "Influenza vaccination", "SEER West", "Multimorbidity group", "A1c screening", "Lipid screening", "Bone density screening", "Psyhcology visit", "Life expectancy over 10 years", "Hispanic", "White race", "Black race", "Metro locality", "SEER South", "SEER Northcentral", "Separated/Divorced/Widowed","Unmarried", "Low value care (sum)", "Radiation Oncologist density", "Diagnostic year 2009", "Diagnostic year 2010", "Diagnostic year 2011", "Diagnostic year 2012", "Diagnostic year 2013", "Diagnostic year 2014" ]

use the list to select a subset of the original DataFrame

$\mathrm{X}=$ dataset[feature_names]

print the first 5 rows

X.head()

\#Instantiate Y with dependent/label var, $X$ with indepdent/feature vars

$\mathrm{y}=$ dataset['trmt']

\#Split data into train and test data sets; 8o/30

from sklearn.model_selection import train_test_split

X_train, X_test, y_train, y_test $=$ train_test_split $(X, y$, test_size $=0.30$, random_state $=21)$

from collections import Counter

\# count \# of examples in each class

counter = Counter $(\mathrm{y})$

\# estimate scale_pos_weight value, assuming the class labels are o and 1. Modify according to your use-case

weight $=$ counter[o] / counter[1]

Calibration before tune

from sklearn.calibration import CalibratedClassifierCV

import seaborn as sns

fig, $a x=$ plt.subplots $(1$, figsize $=(12,6))$

\# Create an uncorrected classifier.

clf $=x g c$

clf.fit(X_train, y_train)

y_test_predict_proba $=$ clf.predict_proba(X_test $)[:, 1]$

fraction_of_positives, mean_predicted_value $=$ calibration_curve(y_test, y_test_predict_proba, n_b ins=10)

plt.plot(mean_predicted_value, fraction_of_positives, 's-', color='red', label='Uncalibrated')

\# Create a corrected classifier.

clf_sigmoid = CalibratedClassifierCV(clf, cv=3, method='sigmoid') 
clf_sigmoid.fit(X_train, y_train)

y_test_predict_proba $=$ clf_sigmoid.predict_proba(X_test) $[:, 1]$

fraction_of_positives, mean_predicted_value = calibration_curve $\left(\mathrm{y} \_t e s t, \mathrm{y}\right.$ _test_predict_proba, $\mathrm{n} \_\mathrm{b}$ ins $=10$ )

plt.plot(mean_predicted_value, fraction_of_positives, 's-', label='Calibrated (Platt)')

plt.plot([0, 1], [0, 1], '--', color='gray')

sns.despine(left=True, bottom=True)

plt.gca().xaxis.set_ticks_position('none')

plt.gca().yaxis.set_ticks_position('none')

plt.gca().legend()

plt.title("\$XGClassifier\$ Sample Calibration Curve", fontsize=20); pass

png

png

from sklearn.metrics import roc_auc_score, accuracy_score, recall_score, precision_score, f1_score, cohen_kappa_score

\# Performance for train

train_y_pred = clf_sigmoid.predict(X_train)

auc $=$ roc_auc_score $\left(y \_\right.$train, train_y_pred)

print("AUC performance for train : ", auc)

\# Calc bal_accuracy, recall, and average positive prediction score

recall $=$ recall_score(y_train, train_y_pred)

print("Recall performance for train : ", recall)

$\mathrm{f} 1=\mathrm{f} 1 \_$score(y_train, train_y_pred)

print("F1 performance for train : ", f1)

prcsn $=$ precision_score $\left(y \_t r a i n\right.$, train_y_pred,labels $=n p$.unique $($ train_y_pred $)$ )

print("Precision performance for train :", presn)

\# Performance for train

y_pred = clf_sigmoid.predict(X_test)

auc_t $=$ roc_auc_score(y_test, $\left.y \_p r e d\right)$

print("AUC performance for test :", auc_t)

\# Calc bal_accuracy, recall, and average positive prediction score

recall_t $=$ recall_score(y_test, $\left.y \_p r e d\right)$

print("Recall performance for test : ", recall_t)

$\mathrm{f} 1 \_\mathrm{t}=\mathrm{f} 1 \_\mathrm{score}\left(\mathrm{y} \_\right.$test, $\mathrm{y} \_$pred $)$

print("F1 performance for test : ", f1_t)

$\operatorname{prcsn} \_\mathrm{t}=$ precision_score $\left(\mathrm{y} \_t\right.$ test, $\mathrm{y} \_$pred,labels $=\mathrm{np}$.unique $\left(\mathrm{y} \_\right.$pred $)$)

print("Precision performance for test : ", prcsn_t)

AUC performance for train : 0.6787989250338197

Recall performance for train : 0.9956783454236765

F1 performance for train : 0.8606497231672336

Precision performance for train : 0.7578712406015038

AUC performance for test : 0.5588647894037931 
Recall performance for test : 0.958139534883721

F1 performance for test : 0.8089412475456879

Precision performance for test : 0.6999477260846837

import xgboost as xgb \# XGBoost

\# Preliminary model $w / o$ tuning or cross-validation

clf_xgb = xgb.XGBClassifier(objective='binary:logistic',scale_pos_weight=weight, seed $=42$ )

clf_xgb.fit(X_train,

y_train,

verbose $=$ True,

\#\# the next three arguments set up early stopping.

early_stopping_rounds $=10$,

eval_metric $=[$ 'auc'],

eval_set $\left.=\left[\left(X \_t e s t, y \_t e s t\right)\right]\right)$

XGBClassifier(base_score $=0.5$, booster='gbtree', colsample_bylevel=1, colsample_bynode $=1$, colsample_bytree $=1$, gamma $=0$, gpu_id $=-1$, importance_type='gain', interaction_constraints=", learning_rate $=0.300000012$, max_delta_step $=0$, max_depth $=6$, min_child_weight $=1$, missing $=$ nan, monotone_constraints='()', n_estimators $=100, n \_j o b s=0$, num_parallel_tree $=1$, random_state $=42$, reg_alpha $=0$, reg_lambda $=1$, scale_pos_weight $=0.4955790381712314$, seed $=42$, subsample $=1$, tree_method='exact', validate_parameters $=1$, verbosity=None)

\# predict probabilities

xgb_probs = clf_xgb.predict_proba(X_test)

\# keep probabilities for the positive outcome only

$\mathrm{xgb} \_$probs $=\mathrm{xgb} \_$probs[:, 1$]$

import matplotlib.pyplot as plt

prediction $=x g b \_$probs

plt.figure $($ figsize $=(15,7))$

plt.hist(prediction[y_test $==0$ ], bins $=50$, label $=$ 'Negatives')

plt.hist(prediction[y_test $==1$ ], bins $=50$, label $=$ 'Positives', alpha $=0.7$, color $=$ 'r')

plt.xlabel('Probability of being Positive Class', fontsize=25)

plt.ylabel('Number of records in each bucket', fontsize $=25$ )

plt.legend(fontsize $=15$ )

plt.tick_params $($ axis $=$ 'both', labelsize $=25$, pad $=5$ )

plt.show()

png

png

\# Performance for train

train_y_pred = clf_xgb.predict(X_train) 
auc $=$ roc_auc_score $\left(y \_\right.$train, train_y_pred $)$

print("AUC performance for train : ", auc)

\# Calc bal_accuracy, recall, and average positive prediction score

recall $=$ recall_score(y_train, train_y_pred)

print("Recall performance for train : ", recall)

$\mathrm{f} 1=\mathrm{f} 1 \_$score(y_train, train_y_pred)

print("F1 performance for train : ", f1)

prcsn $=$ precision_score $\left(y \_t r a i n\right.$, train_y_pred,labels=np.unique(train_y_pred) $)$

print("Precision performance for train : ", prcsn)

\# Performance for train

y_pred = clf_xgb.predict(X_test)

auc_t $=$ roc_auc_score(y_test, $\left.y \_p r e d\right)$

print("AUC performance for test : ", auc_t)

\# Calc bal_accuracy, recall, and average positive prediction score

recall_t $=$ recall_score(y_test, $\left.y \_p r e d\right)$

print("Recall performance for test : ", recall_t)

$\mathrm{f} 1 \_\mathrm{t}=\mathrm{f} 1 \_\mathrm{score}\left(\mathrm{y} \_\right.$test, $\mathrm{y} \_$pred)

print("F1 performance for test : ", f1_t)

prcsn_t $=$ precision_score(y_test, $y \_$pred,labels $=$np.unique(y_pred))

print("Precision performance for test : ", presn_t)

$\mathrm{kp} \_\mathrm{t}=$ cohen_kappa_score(y_test, $\mathrm{y} \_$pred)

print("Cohen Kappa for test : ", kp_t)

AUC performance for train : 0.7268423776363454

Recall performance for train : 0.7471832072850748

F1 performance for train : 0.7892076948157809

Precision performance for train : 0.8362411470029366

AUC performance for test : 0.6110343978606433

Recall performance for test : 0.6715563506261181

F1 performance for test : 0.7101778282255014

Precision performance for test : 0.753512645523886

Cohen Kappa for test : 0.21011514238033613

from sklearn.model_selection import GridSearchCV \# cross validation and tuning from sklearn.model_selection import RepeatedStratifiedKFold

$\mathrm{cv}=$ RepeatedStratifiedKFold(n_splits $=10, \mathrm{n} \_$repeats $=3$, random_state $\left.=1\right)$

\#\# ROUND o

param_grid $=\{$

\}

'scale_pos_weight': [weight]

\#\# NOTE: To speed up cross validiation, and to further prevent overfitting

\#\# we are only using a random subset of the data (90\%) and are only

\#\# using a random subset of the features (columns) (50\%) per tree. 
optimal_params_o = GridSearchCV(

estimator $=x g b . X G B C l a s s i f i e r(o b j e c t i v e='$ binary:logistic',

seed $=42$,

subsample $=0.9$,

param_grid=param_grid,

colsample_bytree $=0.5$ ),

scoring='roc_auc', \#\# see https://scikit-learn.org/stable/modules/model_evaluation.html\#scori

ng-parameter

verbose=0, \# NOTE: If you want to see what Grid Search is doing, set verbose=2

$\mathrm{n} \_$jobs $=-1$,

)

$\mathrm{cr}=\mathrm{cv}$

optimal_params_o.fit(X_train,

y_train,

early_stopping_rounds $=10$,

eval_metric $=[$ 'auc'],

eval_set $=\left[\left(X \_t e s t, y \_t e s t\right)\right]$,

verbose $=$ False)

print(optimal_params_o.best_params_)

\{'scale_pos_weight': 0.4955790381712314$\}$

\# Performance for train

train_y_pred = optimal_params_o.predict(X_train)

auc $=$ roc_auc_score $\left(y \_t r a i n\right.$, train_y_pred)

recall $=$ recall_score $\left(y \_t r a i n, t r a i n \_y \_p r e d\right)$

print("AUC for train : ", auc)

print("Recall for train : ", recall)

AUC for train : 0.6834020319039793

Recall for train : 0.7076709368729742

plot_confusion_matrix(optimal_params_o,

X_test,

y_test,

values_format='d',

display_labels=["No Treatment", "Treatment"])

<sklearn.metrics._plot.confusion_matrix.ConfusionMatrixDisplay at ox1799dd47fao >

png

png

\# Performance for train

train_y_pred = optimal_params_o.predict(X_train)

auc $=$ roc_auc_score(y_train, train_y_pred)

print("AUC performance for train : ", auc)

\# Calc bal_accuracy, recall, and average positive prediction score

recall $=$ recall_score $\left(y \_t r a i n\right.$, train_y_pred $)$ 
print("Recall performance for train : ", recall)

$\mathrm{f} 1=\mathrm{f} 1 \_$score(y_train, train_y_pred)

print("F1 performance for train : ", f1)

prcsn $=$ precision_score $\left(y \_t r a i n\right.$, train_y_pred,labels=np.unique $($ train_y_pred $)$ )

print("Precision performance for train :", presn)

\# Performance for train

y_pred = optimal_params_o.predict(X_test)

auc_t $=$ roc_auc_score(y_test, $\left.y \_p r e d\right)$

print("AUC performance for test : ", auc_t)

\# Calc bal_accuracy, recall, and average positive prediction score

recall_t $=$ recall_score(y_test, $\left.y \_p r e d\right)$

print("Recall performance for test : ", recall_t)

$\mathrm{f} 1 \_\mathrm{t}=\mathrm{f} 1 \_\mathrm{score}\left(\mathrm{y} \_\right.$test, $\mathrm{y} \_$pred $)$

print("F1 performance for test : ", f1_t

$\operatorname{prcsn} \_t=$ precision_score $\left(y \_t e s t, y \_p r e d, l a b e l s=n p . u n i q u e\left(y \_p r e d\right)\right)$

print("Precision performance for test : ", presn_t)

AUC performance for train : 0.6834020319039793

Recall performance for train : 0.7076709368729742

F1 performance for train : 0.7538018906699548

Precision performance for train : 0.8063665142455153

AUC performance for test : 0.5978129739102193

Recall performance for test : 0.643649373881932

F1 performance for test : 0.6911256242796773

Precision performance for test : 0.7461634176690171

\# Use recommended XGboost approach or tune for best performance metric.

weight = optimal_params_o.best_params_['scale_pos_weight']

\#\# ROUND 1

n_estimators $=[20,50,100,200,300,400,500,600,700,800,900]$

learning_rate $=[0.0001,0.001,0.01,0.1,0.2]$

scale_pos_weight $=[$ weight $]$

param_grid_1 = dict(scale_pos_weight=scale_pos_weight, learning_rate=learning_rate,

n_estimators $=$ n_estimators)

$\mathrm{cv}=$ RepeatedStratifiedKFold(n_splits $=10, \mathrm{n} \_$repeats $=3$, random_state $\left.=1\right)$

optimal_params_1 = GridSearchCV(

estimator $=x g b . X G B C l a s s i f i e r(o b j e c t i v e=' b i n a r y: l o g i s t i c '$, seed $=42$,

subsample $=0.9$,

colsample_bytree $=0.5$ ),

param_grid=param_grid_1,

scoring='roc_auc', \#\# see https://scikit-learn.org/stable/modules/model_evaluation.html\#scori ng-parameter 
verbose $=0$, \# NOTE: If you want to see what Grid Search is doing, set verbose $=2$

$$
\begin{aligned}
& \mathrm{n} \_ \text {jobs }=-1, \\
& \mathrm{cv}=\mathrm{cv}
\end{aligned}
$$

)

optimal_params_1.fit(X_train, y_train, early_stopping_rounds $=10$, eval_metric $=[$ 'auc'], eval_set $=\left[\left(X \_\right.\right.$test, $\left.\left.y \_t e s t\right)\right]$, verbose $=$ False)

print(optimal_params_1.best_params_)

\{'learning_rate': 0.01, 'n_estimators': 200, 'scale_pos_weight': 0.4955790381712314$\}$

\# summarize results

print("Best: \%f using \%s" \% (optimal_params_1.best_score_, optimal_params_1.best_params_)) means $=$ optimal_params_1.cv_results_['mean_test_score']

stds = optimal_params_1.cv_results_['std_test_score']

params = optimal_params_1.cv_results_['params']

for mean, stdev, param in zip(means, stds, params):

print("\%f (\%f) with: \%r" \% (mean, stdev, param))

\# Performance for train

train_y_pred $=$ optimal_params_1.predict(X_train)

auc $=$ roc_auc_score $\left(y \_\right.$train, train_y_pred)

print("Performance for train : ", auc)

\# Performance for test

y_pred_rd1 $=$ optimal_params_1.predict(X_test)

auc_t_rd1 = roc_auc_score(y_test, y_pred_rd1)

print("AUC performance for test : ", auc_t_rd1)

\# Calc bal_accuracy, recall, and average positive prediction score

recall_t_rd1 $=$ recall_score(y_test, y_pred_rd1)

print("Recall performance for test : ", recall_t_rd1)

aps_t_rd1 = average_precision_score(y_test, $\left.y \_p r e d\right)$

print("APS performance for test: ", aps_t_rd1)

f1_t_rd1 = f1_score(y_test, $\left.y \_p r e d \_r d 1\right)$

print("F1 performance for test : ", f1_t_rd1)

prcsn_t_rd1 $=$ precision_score(y_test, y_pred_rd1,labels=np.unique(y_pred_rd1)

print("Precision performance for test : ", prcsn_t_rd1)

accry_t $=$ accuracy_score(y_test, $y \_p r e d \_r d 1$ )

print("Accuracy performance for test : ", accry_t)

Performance for train : 0.6850635083482383

AUC performance for test : 0.6114555640824836

Recall performance for test : 0.6826475849731664 
APS performance for test: 0.7196331537267628

F1 performance for test : 0.7158131682611143

Precision performance for test : 0.7523659305993691

Accuracy performance for test : 0.6359048305695746

learning_rate $=$ optimal_params_1.best_params_['learning_rate']

n_estimators $=$ optimal_params_1.best_params_['n_estimators']

\#\# ROUND 2

from sklearn.model_selection import RepeatedStratifiedKFold

param_grid_2 $=\{$

'max_depth': [1,2,3,4,5,6,7,8,9,10,11,12,13,14],

'min_child_weight': [1,2,3,4,5,6,7,8,9,10,11,12,13,14],

'scale_pos_weight': [weight],

'learning_rate':[learning_rate],

\}

'n_estimators':[n_estimators]

$\mathrm{cv}=$ RepeatedStratifiedKFold(n_splits=10, $\mathrm{n} \_$repeats $=3$, random_state $\left.=1\right)$

optimal_params_2= GridSearchCV(

estimator $=x g b . X G B C l a s s i f i e r(o b j e c t i v e=' b i n a r y: l o g i s t i c '$,

seed $=42$,

subsample $=0.9$,

colsample_bytree $=0.5$ ),

param_grid=param_grid_2,

scoring='roc_auc', \#\# see https://scikit-learn.org/stable/modules/model_evaluation.html\#scori

ng-parameter

verbose=3, \# NOTE: If you want to see what Grid Search is doing, set verbose=2

$\mathrm{n} \_$jobs $=-1$,

)

$\mathrm{cV}=\mathrm{cv}$

optimal_params_2.fit(X_train,

y_train,

early_stopping_rounds $=10$,

eval_metric $=[$ 'auc'],

eval_set $=\left[\left(X \_\right.\right.$test, $\left.\left.y \_t e s t\right)\right]$,

verbose $=$ False)

print(optimal_params_2.best_params_)

\{'learning_rate': 0.01, 'max_depth': 12, 'min_child_weight': 10, 'n_estimators': 200, 'scale_pos_weig ht': 0.4955790381712314$\}$

\# Performance for train

train_y_pred = optimal_params_2.predict(X_train)

auc $=$ roc $\_$auc $\_s c o r e\left(y \_t r a i n, t r a i n \_y \_p r e d\right)$

print("Performance for train : ", auc) 
\# Performance for test

y_pred_rd2 = optimal_params_2.predict(X_test)

auc_t_rd2 = roc_auc_score(y_test, y_pred_rd2)

print("AUC performance for test : ", auc_t_rd2)

aps_t_rd2 = average_precision_score(y_test, y_pred_rd2)

print("APS performance for test: ", aps_t_rd2)

\# Calc bal_accuracy, recall, and average positive prediction score

recall_t_rd2 = recall_score(y_test, y_pred_rd2)

print("Recall performance for test : ", recall_t_rd2)

f1_t_rd2=f1_score(y_test, y_pred_rd2)

print("F1 performance for test : ", f1_t_rd2)

prcsn_t_rd2 = precision_score(y_test, $\left.y \_p r e d \_r d 2, l a b e l s=n p . u n i q u e\left(y \_p r e d \_r d 2\right)\right)$

print("Precision performance for test : ", prcsn_t_rd2)

accry_t $=$ accuracy_score(y_test, $\left.y \_p r e d \_r d 2\right)$

print("Accuracy performance for test : ", accry_t)

Performance for train : 0.7176366178513388

AUC performance for test : 0.618159650285361

APS performance for test: 0.7306195103281976

Recall performance for test : 0.6726296958855098

F1 performance for test : 0.7133371276797571

Precision performance for test : 0.7592891760904685

Accuracy performance for test : 0.6368661379476087

min_child_weight $=$ optimal_params_2.best_params_['min_child_weight']

max_depth $=$ optimal_params_2.best_params_['max_depth']

\#\# ROUND 3

param_grid_3 $=\{$

'max_depth': [max_depth],

'min_child_weight': [min_child_weight],

'scale_pos_weight': [weight],

'learning_rate':[learning_rate],

'n_estimators':[n_estimators],

\}

'gamma': [0,0.001,0.002,0.01,0.1,0.2,0.3,0.4,0.5]

$\mathrm{cv}=$ RepeatedStratifiedKFold(n_splits=10, $\mathrm{n} \_$repeats $=3$, random_state $=1$ )

optimal_params_3 = GridSearchCV(

estimator $=x g b . X G B C l a s s i f i e r(o b j e c t i v e='$ binary:logistic',

seed $=42$,

subsample $=0.9$,

colsample_bytree $=0.5$ ),

param_grid=param_grid_3,

scoring='roc_auc', \#\# see https://scikit-learn.org/stable/modules/model_evaluation.html\#scori 


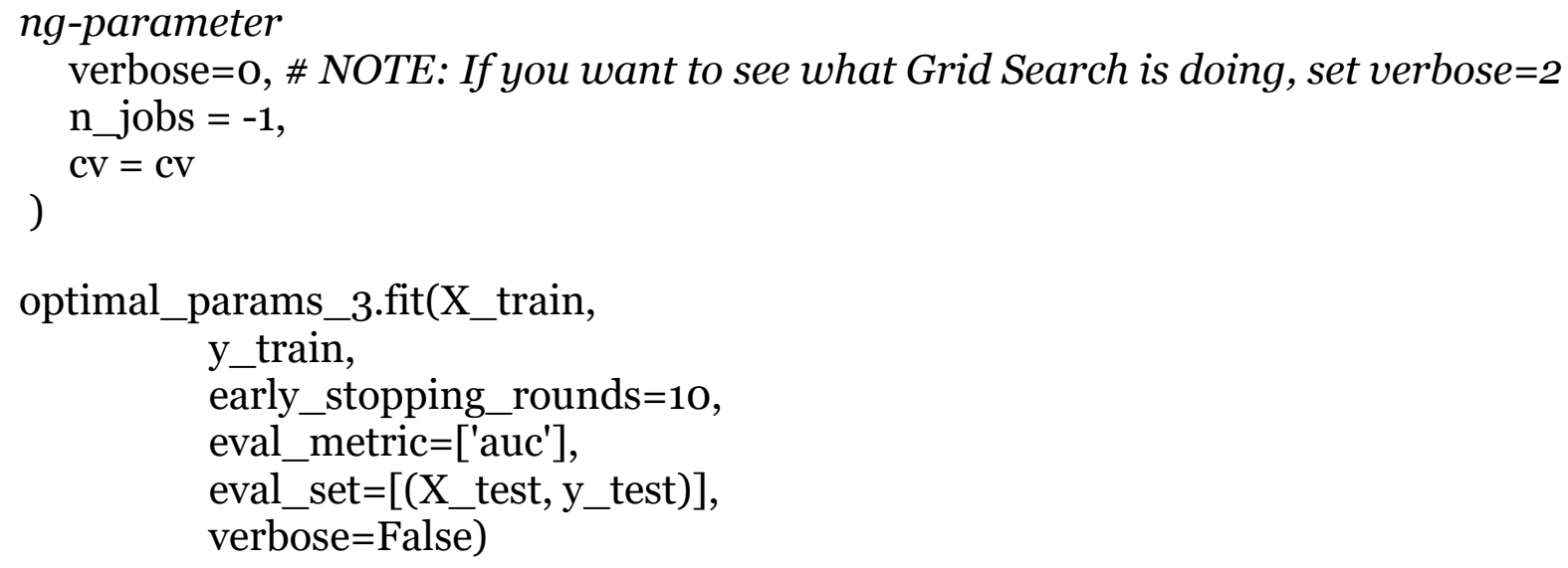

print(optimal_params_3.best_params_)

\{'gamma': 0.01, 'learning_rate': 0.01, 'max_depth': 12, 'min_child_weight': 10, 'n_estimators': 200, 's cale_pos_weight': 0.4955790381712314$\}$

\# Performance for train

train_y_pred = optimal_params_3.predict(X_train)

auc $=$ roc_auc_score $\left(y \_\right.$train, train_y_pred)

print("Performance for train : ", auc)

\section{\# Performance for test}

y_pred_rd3 = optimal_params_3.predict(X_test)

auc_t_rd3 = roc_auc_score(y_test, y_pred_rd3)

print("AUC performance for test : ", auc_t_rd3)

\# average precision score

aps_t_rd3 = average_precision_score(y_test, y_pred_rd3)

print("APS performance for test: ", aps_t_rd3)

\# Calc bal_accuracy, recall, and average positive prediction score

recall_t_rd $3=$ recall_score(y_test, y_pred_rd3)

print("Recall performance for test : ", recall_t_rd3)

f1_t_rd3 = f1_score(y_test, y_pred_rd3)

print("F1 performance for test : ", f1_t_rd3)

prcsn_t_rd3 = precision_score(y_test, $y \_p r e d \_r d 3$,labels=np.unique(y_pred_rd3))

print("Precision performance for test : ", prcsn_t_rd3)

accry_t $=$ accuracy_score(y_test, $\left.y \_p r e d \_r d 3\right)$

print("Accuracy performance for test : ", accry_t)

Performance for train : 0.7176366178513388

AUC performance for test : 0.6183385411619263

APS performance for test: 0.7307162431227368

Recall performance for test : 0.6729874776386404

F1 performance for test : 0.7135811836115326

Precision performance for test : 0.7593863544610416

Accuracy performance for test : 0.6371064647921173 
\# set n_estimaters according to round 3

gamma = optimal_params_3.best_params_['gamma']

\#\# ROUND 4

param_grid_4 $=\{$

'max_depth': [max_depth],

'min_child_weight': [min_child_weight],

'scale_pos_weight': [weight],

'learning_rate':[learning_rate],

'n_estimators':[n_estimators],

'gamma': [gamma],

'reg_lambda':[1,2,3,4,5,10,50],

\}

'reg_alpha':[1,2,3,4,5,10,50]

$\mathrm{cv}=$ RepeatedStratifiedKFold(n_splits=10, $\mathrm{n} \_$repeats $=3$, random_state $\left.=1\right)$

optimal_params_4= GridSearchCV(

estimator=xgb.XGBClassifier(objective='binary:logistic',

seed $=42$,

subsample $=0.9$,

colsample_bytree $=0.5$ ),

param_grid=param_grid_4,

scoring='roc_auc', \#\# see https://scikit-learn.org/stable/modules/model_evaluation.html\#scori ng-parameter

verbose=0, \# NOTE: If you want to see what Grid Search is doing, set verbose=2

$\mathrm{n} \_$jobs $=-1$,

)

$\mathrm{cv}=\mathrm{cv}$

optimal_params_4.fit(X_train,

y_train,

early_stopping_rounds $=10$,

eval_metric=['auc'],

eval_set=[(X_test, y_test $)]$,

verbose $=$ False)

print(optimal_params_4.best_params_)

\{'gamma': 0.01, 'learning_rate': o.01, 'max_depth': 12, 'min_child_weight': 10, 'n_estimators': 200, 'r eg_alpha': 1, 'reg_lambda': 3, 'scale_pos_weight': 0.4955790381712314$\}$

\# Performance for train

train_y_pred $=$ optimal_params_4.predict(X_train)

auc $=$ roc_auc_score $\left(y \_\right.$train, train_y_pred)

print("Performance for train : ", auc)

\# Performance for test

y_pred_rd $4=$ optimal_params_4.predict(X_test)

auc_t_rd4 = roc_auc_score(y_test, y_pred_rd4) 
print("AUC performance for test : ", auc_t_rd4)

\# Calc bal_accuracy, recall, and average positive prediction score

recall_t_rd $4=$ recall_score(y_test, y_pred_rd4)

print("Recall performance for test : ", recall_t_rd4)

\# average precision score

aps_t_rd4 = average_precision_score(y_test, y_pred_rd4)

print("APS performance for test: ", aps_t_rd4)

f1_t_rd4 = f1_score(y_test, y_pred_rd4)

print("F1 performance for test : ", f1_t_rd4)

prcsn_t_rd4 = precision_score(y_test, $y \_p r e d \_r d 4$, labels=np.unique(y_pred_rd4) $)$

print("Precision performance for test : ", prcsn_t_rd4)

accry_t $=$ accuracy_score(y_test, y_pred_rd4)

print("Accuracy performance for test : ", accry_t)

Performance for train : 0.6860186303260307

AUC performance for test : 0.6065291241156949

Recall performance for test : 0.6654740608228981

APS performance for test: 0.7242139745672891

F1 performance for test : 0.7054807509956381

Precision performance for test : 0.7506053268765133

Accuracy performance for test : 0.6267724104782504

reg_lambda $=$ optimal_params_4.best_params_['reg_lambda']

reg_alpha $=$ optimal_params_4.best_params_['reg_alpha']

\#\# ROUND 5

param_grid_ $5=\{$

'max_depth': [max_depth],

'min_child_weight': [min_child_weight],

'scale_pos_weight': [weight],

'learning_rate':[learning_rate],

'n_estimators':[n_estimators],

'gamma': [gamma],

'reg_lambda':[reg_lambda],

'reg_alpha':[reg_alpha],

'subsample':[0.3,0.5,0.7,0.9],

\}

'colsample_bytree':[0.3,0.5,0.7,0.9]

$\mathrm{cv}=$ RepeatedStratifiedKFold(n_splits=10, $\mathrm{n} \_$repeats $=3$, random_state $=1$ )

optimal_params_5 = GridSearchCV(

estimator $=x g b . X G B C l a s s i f i e r(o b j e c t i v e=' b i n a r y: l o g i s t i c '$,

seed $=42$

)

param_grid=param_grid $\_5$,

scoring='roc_auc', \#\# see https://scikit-learn.org/stable/modules/model_evaluation.html\#scori 


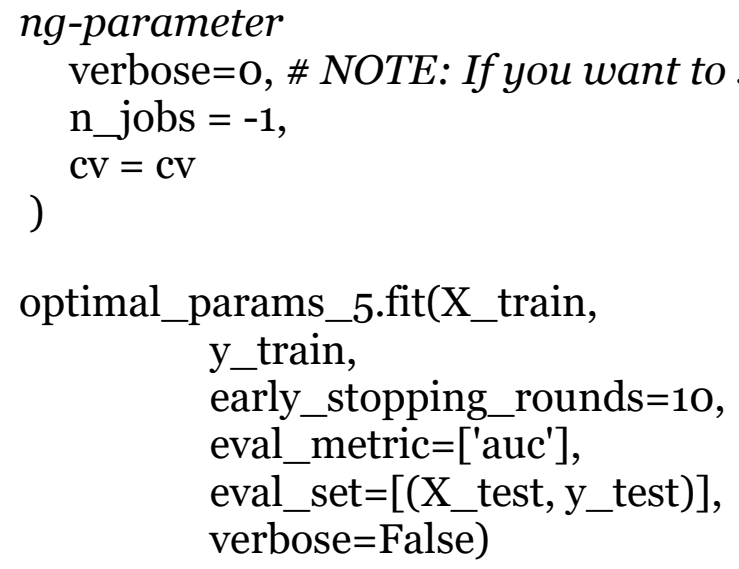

print(optimal_params_5.best_params_)

\{'colsample_bytree': 0.5, 'gamma': o.01, 'learning_rate': 0.01, 'max_depth': 12, 'min_child_weight': 1 o, 'n_estimators': 200, 'reg_alpha': 1, 'reg_lambda': 3, 'scale_pos_weight': 0.4955790381712314 , 'sub sample': 0.9\}

\# Performance for train

train_y_pred = optimal_params_5.predict(X_train)

auc $=$ roc_auc_score $\left(y \_\right.$train, train_y_pred $)$

print("Performance for train : ", auc)

\# Performance for test

y_pred_rd5 $=$ optimal_params_5.predict(X_test)

auc_t_rd5 = roc_auc_score(y_test, y_pred_rd5)

print("AUC performance for test : ", auc_t_rd5)

\# average precision score

aps_t_rd5 = average_precision_score(y_test, y_pred_rd5)

print("APS performance for test: ", aps_t_rd5)

\# Calc bal_accuracy, recall, and average positive prediction score

recall_t_rd5 = recall_score(y_test, y_pred_rd5)

print("Recall performance for test : ", recall_t_rd5)

f1_t_rd5 = f1_score(y_test, y_pred_rd5)

print("F1 performance for test : ", f1_t_rd5)

prcsn_t_rd5 = precision_score(y_test, y_pred_rd5,labels=np.unique(y_pred_rd5))

print("Precision performance for test : ", prcsn_t_rd5)

accry_t $=$ accuracy_score(y_test, $\left.y \_p r e d \_r d 5\right)$

print("Accuracy performance for test : ", accry_t)

Performance for train : 0.6860186303260307

AUC performance for test : 0.6065291241156949

APS performance for test: 0.7242139745672891

Recall performance for test : 0.6654740608228981

F1 performance for test : 0.7054807509956381

Precision performance for test : 0.7506053268765133

Accuracy performance for test : 0.6267724104782504 
subsample = optimal_params_5.best_params_['subsample']

colsample_bytree = optimal_params_5.best_params_['colsample_bytree']

\#\# ROUND 6

param_grid_6 $=\{$

'max_depth': [max_depth],

'min_child_weight': [min_child_weight],

'scale_pos_weight': [weight],

'learning_rate':[learning_rate],

'n_estimators':[n_estimators],

'gamma': [gamma],

'reg_lambda':[reg_lambda],

'reg_alpha':[reg_alpha],

'subsample':[subsample],

\}

'colsample_bytree':[colsample_bytree]

$\mathrm{cv}=$ RepeatedStratifiedKFold(n_splits=10, $\mathrm{n} \_$repeats $=3$, random_state $\left.=1\right)$

optimal_params_6 = GridSearchCV(

estimator=xgb.XGBClassifier(objective='binary:logistic', seed $=42$ ),

param_grid=param_grid_6,

scoring='roc_auc', \#\# see https://scikit-learn.org/stable/modules/model_evaluation.html\#scori ng-parameter

verbose=0, \# NOTE: If you want to see what Grid Search is doing, set verbose=2

$\mathrm{n} \_$jobs $=-1$,

)

$\mathrm{cv}=\mathrm{cv}$

optimal_params_6.fit(X_train,

y_train,

early_stopping_rounds $=10$,

eval_metric $=[$ 'auc'],

eval_set=[(X_test, y_test $)]$,

verbose $=$ False)

print(optimal_params_6.best_params_)

\{'colsample_bytree': 0.5, 'gamma': 0.01, 'learning_rate': 0.01, 'max_depth': 12, 'min_child_weight': 1 o, 'n_estimators': 200, 'reg_alpha': 1, 'reg_lambda': 3, 'scale_pos_weight': 0.4955790381712314 , 'sub sample': 0.9\}

$\operatorname{print}\left(f^{\prime} B e s t:\{\right.$ optimal_params_6.best_score_\} using \{optimal_params_6.best_params_\}','\n')

Best: 0.6860864943064999 using \{'colsample_bytree': 0.5, 'gamma': 0.01, 'learning_rate': 0.01, 'max _depth': 12, 'min_child_weight': 10, 'n_estimators': 200, 'reg_alpha': 1, 'reg_lambda': 3, 'scale_pos_ weight': 0.4955790381712314 , 'subsample': 0.9$\}$

from sklearn.model_selection import cross_validate 
xgbcl = optimal_params_6.best_estimator_

kfold $=$ RepeatedStratifiedKFold(n_splits=10, n_repeats=3, random_state=1)

\#refit the model on $k$-folds to get stable avg error metrics

scores $=$ cross_validate(estimator $=x g b c l, X=X \_t r a i n, y=y \_t r a i n, c v=k f o l d, n \_j o b s=-1$, scoring=['accuracy', 'roc_auc','average_precision','precision', 'recall', 'f1','neg_log_loss'])

print('Training 5-fold Cross Validation Results: $\backslash \mathrm{n}$ ')

print('AUC: ', scores['test_roc_auc'].mean())

print('Average precision: ', scores['test_average_precision'].mean())

print('Accuracy: ', scores['test_accuracy'].mean())

print('Precision: ', scores['test_precision'].mean())

print('Recall: ', scores['test_recall'].mean())

print('F1: ', scores['test_f1'].mean())

print('Neg.LogLoss: ', scores['test_neg_log_loss'].mean(), '\n')

Training 5 -fold Cross Validation Results:

AUC: 0.6876457369330226

Average precision: 0.7995369612229754

Accuracy: 0.6535853497120976

Precision: 0.763820977300308

Recall: 0.6962997945567067

F1: 0.7284155332975544

Neg.LogLoss: -0.6369035750460006

AUC: 0.6921795663616259 Accuracy: 0.7015148941290553 Precision: 0.7124730885535536 Recall:

0.926738078882592 F1: 0.8055823014055182 Neg.LogLoss: -0.579314435521734

import sklearn.metrics as metrics

\#Fit the final model

xgbcl.fit(X_train, y_train)

\#Generate predictions against our training and test data

pred_train $=$ xgbcl.predict $\left(X \_\right.$train $)$

proba_train $=$ xgbcl.predict_proba $\left(X \_t r a i n\right)$

pred_test $=$ xgbcl.predict $\left(\mathrm{X} \_\right.$test $)$

proba_test $=$ xgbcl.predict_proba $\left(X \_\right.$test $)$

\# Print model report

print("Classification report (Test): \n")

print(metrics.classification_report(y_test, pred_test))

print("Confusion matrix (Test): $\backslash \mathrm{n} ")$

$\operatorname{print}($ metrics.confusion_matrix(y_test, pred_test)/len(y_test))

print ('\nTrain Accuracy:', metrics.accuracy_score(y_train, pred_train))

print ('Test Accuracy:', metrics.accuracy_score(y_test, pred_test))

print ('\nTrain AUC:', metrics.roc_auc_score(y_train, proba_train[:,1]))

print ('Test AUC:', metrics.roc_auc_score(y_test, proba_test[:,1])) 
\# calculate the fpr and tpr for all thresholds of the classification

train_fpr, train_tpr, train_threshold = metrics.roc_curve(y_train, proba_train[:,1])

test_fpr, test_tpr, test_threshold = metrics.roc_curve(y_test, proba_test[:,1])

train_roc_auc $=$ metrics.auc(train_fpr, train_tpr)

test_roc_auc $=$ metrics.auc(test_fpr, test_tpr)

import matplotlib.pyplot as plt

fig, ax $=$ plt.subplots(figsize $=[7,5]$ )

plt.title('Receiver Operating Characteristic')

plt.plot(train_fpr, train_tpr, 'b', label = 'Train AUC = \%o.2f' \% train_roc_auc)

plt.plot(test_fpr, test_tpr, 'g', label $=$ 'Test AUC $=\% 0.2 f^{\prime} \%$ test_roc_auc)

plt.legend(loc $=$ 'lower right')

plt.plot([0, 1], [o, 1],'r--')

plt.xlim([o, 1])

plt.ylim([o, 1])

plt.ylabel('True Positive Rate')

plt.xlabel('False Positive Rate')

plt.show()

\# plot feature importance

xgb.plot_importance(xgbcl, importance_type='gain');

Classification report (Test):

precision recall f1-score support
0.0
0.44
0.55
$0.49 \quad 1366$
1.0
0.75
0.67
$0.71 \quad 2795$

accuracy

macro avg

$\begin{array}{llll} & & 0.63 & 4161 \\ 0.60 & 0.61 & 0.60 & 4161\end{array}$

$\begin{array}{lllll}\text { weighted avg } & 0.65 & 0.63 & 0.63 & 4161\end{array}$

Confusion matrix (Test):

[[0.17976448 0.14852199$]$

$\left[\begin{array}{lll}0.2247056 & 0.44700793\end{array}\right]$

Train Accuracy: 0.6966731898238747

Test Accuracy: 0.6267724104782504

Train AUC: 0.755213509518965

Test AUC: 0.6567936626008062

png

png

png

png 
from sklearn.calibration import CalibratedClassifierCV

fig, ax = plt.subplots $(1$, figsize $=(12,6))$

\# Create an uncorrected classifier.

$\mathrm{clf}=\mathrm{xgc}$

clf.fit(X_train, y_train)

y_test_predict_proba $=$ clf.predict_proba(X_test) $[:, 1]$

fraction_of_positives, mean_predicted_value = calibration_curve $\left(\mathrm{y} \_t\right.$ test, $\mathrm{y} \_$test_predict_proba, $\mathrm{n} \_\mathrm{b}$ ins $=10$ )

plt.plot(mean_predicted_value, fraction_of_positives, 's-', color='red', label='Uncalibrated')

\# Create a corrected classifier.

clf_sigmoid $=$ CalibratedClassifierCV(clf, cv=3, method='sigmoid')

clf_sigmoid.fit(X_train, y_train)

y_test_predict_proba $=$ clf_sigmoid.predict_proba(X_test) $[:, 1]$

fraction_of_positives, mean_predicted_value = calibration_curve $\left(\mathrm{y} \_t\right.$ test, $\mathrm{y} \_t$ test_predict_proba, $\mathrm{n} \_\mathrm{b}$ ins $=10$ )

plt.plot(mean_predicted_value, fraction_of_positives, 's-', label='Calibrated (Platt)')

plt.plot([0, 1], [0, 1], '--', color='gray')

\# Create a corrected classifier.

clf_sigmoid_tune $=$ CalibratedClassifierCV $(\mathrm{xgbcl}, \mathrm{cv}=3$, method='sigmoid')

clf_sigmoid_tune.fit(X_train, y_train)

y_test_predict_proba $=$ clf_sigmoid_tune.predict_proba(X_test)[:, 1$]$

fraction_of_positives, mean_predicted_value = calibration_curve $\left(\mathrm{y} \_t e s t, \mathrm{y} \_t\right.$ test_predict_proba, $\mathrm{n} \_\mathrm{b}$ ins $=10$ )

plt.plot(mean_predicted_value, fraction_of_positives, 's-', label='Calibrated (Platt), After tune')

plt.plot([0, 1], [0, 1], '--', color='green')

sns.despine(left=True, bottom=True)

plt.gca().xaxis.set_ticks_position('none')

plt.gca().yaxis.set_ticks_position('none')

plt.gca().legend()

plt.title("\$XGClassifier\$ Sample Calibration Curve", fontsize=20); pass

png

png

import sklearn.metrics as metrics

\#Fit the final model

clf_sigmoid_tune.fit(X_train, y_train)

\#Generate predictions against our training and test data

pred_train = clf_sigmoid_tune.predict(X_train)

proba_train $=$ clf_sigmoid_tune.predict_proba $\left(X \_t r a i n\right)$

pred_test $=$ clf_sigmoid_tune.predict(X_test)

proba_test $=$ clf_sigmoid_tune.predict_proba(X_test) 
\# Print model report

print("Classification report (Test): \n")

print(metrics.classification_report(y_test, pred_test))

print("Confusion matrix (Test): \n")

print(metrics.confusion_matrix(y_test, pred_test)/len(y_test))

print ('\nTrain Accuracy:', metrics.accuracy_score(y_train, pred_train))

print ('Test Accuracy:', metrics.accuracy_score(y_test, pred_test))

print ('\nTrain AUC:', metrics.roc_auc_score(y_train, proba_train[:,1]))

print ('Test AUC:', metrics.roc_auc_score(y_test, proba_test[:,1]))

print ('\nTrain PR-AUC:', metrics.average_precision_score(y_train, proba_train[:,1]))

print ('Test PR-AUC:', metrics.average_precision_score(y_test, proba_test[:,1]))

\# calculate the fpr and tpr for all thresholds of the classification

train_fpr, train_tpr, train_threshold = metrics.roc_curve(y_train, proba_train[:,1])

test_fpr, test_tpr, test_threshold = metrics.roc_curve(y_test, proba_test[:,1])

train_roc_auc $=$ metrics.auc $($ train_fpr, train_tpr)

test_roc_auc $=$ metrics.auc(test_fpr, test_tpr)

import matplotlib.pyplot as plt

fig, ax $=$ plt.subplots(figsize $=[7,5])$

plt.title('Receiver Operating Characteristic')

plt.plot(train_fpr, train_tpr, 'b', label = 'Train AUC = \%o.2f' \% train_roc_auc)

plt.plot(test_fpr, test_tpr, ' $\mathrm{g}$ ', label $=$ 'Test AUC $=\% 0.2 \mathrm{f}^{\prime} \%$ test_roc_auc)

plt.legend $($ loc $=$ 'lower right')

plt.plot([o, 1], [o, 1],'r--')

plt.xlim([o, 1])

plt.ylim([0, 1])

plt.ylabel('True Positive Rate')

plt.xlabel('False Positive Rate')

plt.show()

\# plot feature importance

xgb.plot_importance(xgbcl, importance_type='gain');

Classification report (Test):

precision recall f1-score support

$\begin{array}{lllll}0.0 & 0.60 & 0.23 & 0.33 & 1366\end{array}$

$\begin{array}{lllll}1.0 & 0.71 & 0.92 & 0.80 & 2795\end{array}$

accuracy

macro avg

weighted avg
$\begin{array}{llll}0.65 & 0.58 & 0.57 & 4161\end{array}$
$\begin{array}{llll}0.67 & 0.70 & 0.65 & 4161\end{array}$ 
Confusion matrix (Test):

[[0.0752223 0.25306417$]$

[0.05118962 0.62052391]

Train Accuracy: 0.7289113193943764

Test Accuracy: 0.695746214852199

Train AUC: 0.7814297155324872

Test AUC: 0.6598860127240391

Train PR-AUC: 0.8725053236929292

Test PR-AUC: 0.7798344142467664

\# predicted probabilities using tuning and calibration curve

final_model = clf_sigmoid_tune.fit(X_train, np.ravel(y_train))

predictions $=$ final_model.predict(X_test)

preds = final_model.predict_proba(X_test)

\# Create a dataframe for the probabilities of treatment

preds_df $=$ pd.DataFrame(preds[:,1], columns $=$ ['prob_treatment'])

print("Accuracy Score Before and After Thresholding: \{\}$,\{\}$ ".format(accuracy_score(y_test, predictio ns), accuracy_score(y_test, roc_predictions)))

print("Precision Score Before and After Thresholding: \{\}$,\{\}$ ".format(precision_score(y_test, predictio ns), precision_score(y_test, roc_predictions)))

print("Recall Score Before and After Thresholding: \{\}$,\{\}$ ".format(recall_score(y_test, predictions), re call_score(y_test, roc_predictions)) )

print("F1 Score Before and After Thresholding: \{\}$,\{\} "$.format(f1_score(y_test, predictions), f1_score( y_test, roc_predictions)))

print("Roc Score Before and After Thresholding: \{\}$,\{\}$ ".format(roc_auc_score(y_test, predictions), ro c_auc_score(y_test, roc_predictions))

Accuracy Score Before and After Thresholding: 0.695746214852199, o.6534486902186974

Precision Score Before and After Thresholding: 0.7103163686382393, 0.7420393559928443

Recall Score Before and After Thresholding: 0.9237924865831842, 0.7420393559928443

F1 Score Before and After Thresholding: 0.8031104199066873, 0.7420393559928443

Roc Score Before and After Thresholding: 0.5764643252828073, 0.6071104539847092

from sklearn.calibration import CalibratedClassifierCV

fig, ax = plt.subplots $(1$, figsize $=(12,6))$

\# Create an uncorrected classifier.

clf $=$ xgbcl

clf.fit(X_train, y_train)

y_test_predict_proba $=$ clf.predict_proba $\left(X \_t e s t\right)[:, 1]$

fraction_of_positives, mean_predicted_value = calibration_curve $\left(\mathrm{y} \_t e s t, \mathrm{y}\right.$ _test_predict_proba, $\mathrm{n} \_\mathrm{b}$ ins $=10$ )

plt.plot(mean_predicted_value, fraction_of_positives, 's-', color='red', label='Uncalibrated') 
\# Create a corrected classifier.

clf_sigmoid $=$ CalibratedClassifierCV(clf, cv=3, method='sigmoid')

clf_sigmoid.fit(X_train, y_train)

y_test_predict_proba $=$ clf_sigmoid.predict_proba(X_test $)[:, 1]$

fraction_of_positives, mean_predicted_value = calibration_curve $\left(\mathrm{y} \_t\right.$ test, $\mathrm{y} \_t$ test_predict_proba, $\mathrm{n} \_\mathrm{b}$ ins $=10$ )

plt.plot(mean_predicted_value, fraction_of_positives, 's-', label='Calibrated (Platt)')

plt.plot([0, 1], [0, 1], '--', color='gray')

\# Create a corrected classifier.

clf_sigmoid_tune $=$ CalibratedClassifierCV(optimal_params_6.best_estimator_, cv=3, method='sig moid')

clf_sigmoid_tune.fit(X_train, y_train)

y_test_predict_proba $=$ clf_sigmoid_tune.predict_proba(X_test)[:, 1]

fraction_of_positives, mean_predicted_value = calibration_curve $\left(\mathrm{y} \_t\right.$ test, $\mathrm{y}$ _test_predict_proba, $\mathrm{n} \_\mathrm{b}$ ins $=10$ )

plt.plot(mean_predicted_value, fraction_of_positives, 's-', label='Calibrated (Platt), After tune')

plt.plot([0, 1], [0, 1], '--', color='green')

\# Create a corrected classifier.

fraction_of_positives, mean_predicted_value = calibration_curve $\left(\mathrm{y} \_t e s t\right.$, roc_predictions, $\mathrm{n} \_b i n s=1$ o)

plt.plot(mean_predicted_value, fraction_of_positives, 's-', label='Calibrated (Platt), After tune \& thre shold')

plt.plot([0, 1], [0, 1], '--', color='yellow')

sns.despine(left=True, bottom=True)

plt.gca().xaxis.set_ticks_position('none')

plt.gca().yaxis.set_ticks_position('none')

plt.gca().legend()

plt.title("\$XGClassifier\$ Sample Calibration Curve", fontsize=20); pass

png

png

import shap

shap_values_list $=[]$

shap_values_list_1000 $=[]$

for calibrated_classifier in clf_sigmoid_tune.calibrated_classifiers_:

explainer $=$ shap.TreeExplainer(calibrated_classifier.base_estimator)

shap_values $=$ explainer.shap_values(X_train)

shap_values_1000 $=$ explainer.shap_values(X_test[:100o])

shap_values_list_1000.append(shap_values_1000)

shap_values_list.append(shap_values)

shap_values $=$ np.array $\left(\operatorname{shap} \_v a l u e s \_l i s t\right) . s u m($ axis $=0) /$ len $\left(\operatorname{shap} \_v a l u e s \_l i s t\right)$

First summary plot is tune 
\# Shap values for positive class

shap.summary_plot(shap_values, X_train, max_display=15, cmap=plt.get_cmap("cool"))

\# Shap values for positive class

shap.summary_plot(shap_values, X_train, max_display=15, plot_type="bar", cmap=plt.get_cmap(" cool"))

\# Shap values for positive class

shap.summary_plot(shap_values, X_train, max_display=50,plot_type="bar", cmap=plt.get_cmap(" cool"))

shap.dependence_plot("Married", shap_values, X_train.values, feature_names=feature_names,inter action_index=None, cmap=plt.get_cmap("cool"))

shap.dependence_plot("Age group 66-74", shap_values, X_train.values, feature_names=feature_na mes,interaction_index=None, cmap=plt.get_cmap("cool"))

shap.dependence_plot("Care fragmentation", shap_values, X_train.values, feature_names=feature_ names,interaction_index=None, cmap=plt.get_cmap("cool"))

shap.dependence_plot("Median income", shap_values, X_train.values, feature_names=feature_nam es,interaction_index=None, cmap=plt.get_cmap("cool"))

shap.dependence_plot("Life expectancy over 10 years", shap_values, X_train.values, feature_names= feature_names,interaction_index=None, cmap=plt.get_cmap("cool"))

shap.dependence_plot("Multimorbidity group", shap_values, X_train.values, feature_names=feature _names,interaction_index=None, cmap=plt.get_cmap("cool")) 
Appendix 7.8 Aim 3 python codes

import pandas as pd

import numpy as np

from scipy.stats import stats, randint

import random

from sklearn.model_selection import GridSearchCV, cross_val_score, learning_curve

from sklearn.model_selection import train_test_split

import xgboost as xgb

from collections import Counter

import itertools

import matplotlib.pyplot as plt

import numpy as np

import seaborn as sns

X_vars $=[$

"Age at diagnosis",

"Hispanic",

"Metro locality",

"Physical condition total","Mental health condition total",

"A1c screening","Influenza vaccination","Lipid screening",

"SEER Northcentral","SEER Northeast","SEER South","SEER West",

"White race","Black race","Other race",

"Psyhcology visit","Primary Care visit",

"Median income",

"Urologist density","Radiation Oncologist density",

"College education","Less than highschool education",

"PCCI",

"Married","Separated/Divorced/Widowed","Unmarried","Marital status unknown",

"Low-risk prostate cancer",

"Low value care (sum)",

"Conservative management",

"Care fragmentation",

"Diagnostic year 2005",

'Diagnostic year 2006',

'Diagnostic year 2007',

'Diagnostic year 2008',

'Diagnostic year 2009',

'Diagnostic year 2010',

'Diagnostic year 2011',

'Diagnostic year 2012',

'Diagnostic year 2013',

'Diagnostic year 2014'

]

\# use the list to select a subset of the original DataFrame

$\mathrm{X}=$ dataset[X_vars] 
$\mathrm{y}=\operatorname{dataset}[$ 'notx_12_24maft']

y.head()

o $\quad 3404.767935$

$1 \quad 1613.494700$

$2 \quad 54756.131876$

$3 \quad 143.520000$

$4 \quad 3225.969687$

Name: notx_12_24maft, dtype: float64

import matplotlib.pyplot as plt

import seaborn as sns; sns.set()

\section{\#Organize Data}

SR_y = pd.Series(y, name="y (Target Vector Distribution)")

\section{\#Plot Data}

fig, ax = plt.subplots()

sns.distplot(SR_y, bins=25, color $=" g ", a x=a x)$

plt.show()

png

png

\#X_train, $y \_t r a i n$

XGB regressor with grid search

import numpy as np

import pandas as pd

from sklearn import preprocessing

import xgboost as xgb

from xgboost.sklearn import XGBRegressor

import datetime

from sklearn.model_selection import GridSearchCV

from sklearn.metrics import r2_score

y_train_full $=$ dataset['notx_12_24maft']

x_train_full $=$ dataset.drop $(["$ notx_12_24maft"] , axis=1)

y_train_full = np.log1p $\left(y \_t r a i n \_f u l l\right)$

Confirm y_ln distribution

import matplotlib.pyplot as plt

import seaborn as sns; sns.set()

\#Organize Data

SR_y_ln = pd.Series(y_train_full, name="y (Target Vector Distribution)")

\#Plot Data

fig, ax = plt.subplots() 
sns.distplot(SR_y_ln, bins=25, color="g", ax=ax)

plt.show()

png

png

\# Split, check distribution of train/test features

from sklearn.model_selection import train_test_split

$\mathrm{X} \_$train, $\mathrm{X} \_$test, $y \_$train, $y \_$test $=$train_test_split(x_train_full, $y \_t r a i n \_f u l l, t e s t \_s i z e=0.3$, random_ state $=1$ )

print(X_train.shape)

print(X_test.shape)

print(y_train.shape)

print(y_test.shape)

$(52969,45)$

$(22702,45)$

$(52969$,

(22702,)

import xgboost as xgb

from sklearn.metrics import r2_score, mean_squared_error, mean_absolute_error

\#set cross-validation and defaults

$\mathrm{cv}=10$

seed $=33$

\#

n_estimators $=[500,670,675]$

learning_rate $=[0.008,0.009,0.01]$

param_grid_1 = dict(learning_rate=learning_rate, n_estimators $=n \_$_estimators)

xgb_model $=$ XGBRegressor $($ objective $=$ 'reg:squarederror', seed=seed)

optimal_params_1 $=$ GridSearchCV(

estimator $=x g b \_$model,

param_grid = param_grid_1,

scoring = 'r2', \#R2 tune

\#scoring = 'neg_mean_squared_error', \#MSE

$\mathrm{cv}=\mathrm{cv}$,

$\mathrm{n} \_$jobs $=-1$,

verbose $=1$

)

optimal_params_1.fit(X_train,

y_train,

early_stopping_rounds $=10$,

eval_set=[(X_test, y_test $)]$, 


$$
\text { verbose }=\text { False) }
$$

print("Learning rate: ", optimal_params_1.best_params_['learning_rate'])

print("n_estimators: ",optimal_params_1.best_params_['n_estimators'])

Fitting 10 folds for each of 9 candidates, totalling 90 fits

[Parallel(n_jobs=-1)]: Using backend LokyBackend with 12 concurrent workers.

[Parallel(n_jobs=-1)]: Done 26 tasks | elapsed: $3.8 \mathrm{~min}$

[Parallel(n_jobs=-1)]: Done 90 out of 90 | elapsed: 10.1min finished

Learning rate: 0.009

n_estimators: 670

\# summarize results

print("Best: \%f using \%s" \% (optimal_params_1.best_score_,optimal_params_1.best_params_)) means $=$ optimal_params_1.cv_results_['mean_test_score']

stds = optimal_params_1.cv_results_['std_test_score']

params = optimal_params_1.cv_results_['params']

for mean, stdev, param in zip(means, stds, params):

print("\%f (\%f) with: \%r" \% (mean, stdev, param))

Best: 0.344432 using \{'learning_rate': 0.009, 'n_estimators': 670\}

0.339254 (0.025079) with: \{'learning_rate': 0.008, 'n_estimators': 500\}

0.344367 (0.024457) with: \{'learning_rate': o.008, 'n_estimators': 670\}

0.344394 (o.024453) with: \{'learning_rate': 0.008, 'n_estimators': 675$\}$

0.342533 (0.024821) with: \{'learning_rate': 0.009, 'n_estimators': 500\}

0.344432 (o.024475) with: \{'learning_rate': 0.009, 'n_estimators': 670\}

o.344430 (0.024477) with: \{'learning_rate': 0.009, 'n_estimators': 675\}

0.343783 (0.024534) with: \{'learning_rate': 0.01, 'n_estimators': 500\}

0.344329 (0.024393) with: \{'learning_rate': o.01, 'n_estimators': 670\}

0.344329 (o.024393) with: \{'learning_rate': 0.01, 'n_estimators': 675\}

y_train_pred = optimal_params_1.best_estimator_.predict(X_train)

y_test_pred = optimal_params_1.best_estimator_.predict(X_test)

print('XGBregressor evaluating result:')

print("Train MAE: ", sklearn.metrics.mean_absolute_error(y_train, y_train_pred))

print("Train RMSE: ", np.sqrt(sklearn.metrics.mean_squared_error(y_train, y_train_pred)))

print("Train R2: ", np.sqrt(sklearn.metrics.r2_score(y_train, y_train_pred)))

print("Test MAE: ", sklearn.metrics.mean_absolute_error(y_test, y_test_pred))

print("Test RMSE: ", np.sqrt(sklearn.metrics.mean_squared_error(y_test, y_test_pred)))

print("Test R2: ", np.sqrt(sklearn.metrics.r2_score(y_test, y_test_pred)))

XGBregressor evaluating result:

Train MAE: 1.1343200343381408

Train RMSE: 1.5691095294270383

Train R2: 0.6387264523125636

Test MAE: 1.1727194767606826 
Test RMSE: 1.627370752580571

Test R2: 0.5810544023298005

learning_rate $=$ optimal_params_1.best_params_['learning_rate']

n_estimators $=$ optimal_params_1.best_params_['n_estimators']

param_grid_2 = \{'learning_rate':[learning_rate],

'n_estimators':[n_estimators],

'max_depth':[4,5,6],

'min_child_weight':[11,12,13]

\}

xgb_model $=$ XGBRegressor $($ objective $=$ 'reg:squarederror', seed=seed)

optimal_params_2 = GridSearchCV(

estimator $=x g b \_$model,

param_grid = param_grid_2,

scoring = 'r2',

\#scoring = 'neg_mean_absolute_error', \#MAE

\#scoring = 'neg_mean_squared_error', \#MSE

$\mathrm{cv}=\mathrm{cv}$,

$\mathrm{n} \_$jobs $=-1$,

verbose $=1$

)

optimal_params_2.fit(X_train,

y_train,

early_stopping_rounds $=10$,

eval_set $=\left[\left(X \_\right.\right.$test, $\left.\left.y \_t e s t\right)\right]$,

verbose $=$ False)

print("max_depth: ", optimal_params_2.best_params_['max_depth'])

print("min_child_weight: ",optimal_params_2.best_params_['min_child_weight'])

Fitting 10 folds for each of 9 candidates, totalling 90 fits

[Parallel(n_jobs=-1)]: Using backend LokyBackend with 12 concurrent workers.

[Parallel(n_jobs=-1)]: Done 26 tasks | elapsed: 2.9min

[Parallel(n_jobs=-1)]: Done 90 out of 90 | elapsed: $9.2 \mathrm{~min}$ finished

max_depth: 5

min_child_weight: 12

max_depth = optimal_params_2.best_params_['max_depth']

min_child_weight $=$ optimal_params_2.best_params_['min_child_weight']

y_train_pred $=$ optimal_params_2.best_estimator_.predict(X_train)

y_test_pred = optimal_params_2.best_estimator_.predict(X_test) 
print('XGBregressor evaluating result:')

print("Train MAE: ", sklearn.metrics.mean_absolute_error(y_train, y_train_pred))

print("Train RMSE: ", np.sqrt(sklearn.metrics.mean_squared_error(y_train, y_train_pred)))

print("Train R2: ", np.sqrt(sklearn.metrics.r2_score(y_train, y_train_pred)))

print("Test MAE: ", sklearn.metrics.mean_absolute_error(y_test, y_test_pred))

print("Test RMSE: ", np.sqrt(sklearn.metrics.mean_squared_error(y_test, y_test_pred)))

print("Test R2: ", np.sqrt(sklearn.metrics.r2_score(y_test, y_test_pred)))

XGBregressor evaluating result:

Train MAE: 1.1557842730348065

Train RMSE: 1.6082375631598405

Train R2: 0.6148797875288853

Test MAE: 1.1723220292261036

Test RMSE: 1.6260654683448836

Test R2: 0.5819676548322498

\section{\# gamma tune}

param_grid_3 = \{'learning_rate':[learning_rate],

'n_estimators':[n_estimators],

'max_depth':[max_depth],

'min_child_weight':[min_child_weight],

'gamma':[0.003,0.004,0.005,0.006,0.007] \}

xgb_model $=$ XGBRegressor $($ objective $=$ 'reg:squarederror', seed $=$ seed)

optimal_params_3 = GridSearchCV(

estimator $=x g b \_$model,

param_grid $=$ param_grid $\_3$,

scoring = 'r2', \#MAE

\#scoring = 'neg_mean_squared_error', \#MSE

$\mathrm{cv}=\mathrm{cv}$,

$\mathrm{n} \_$jobs $=-1$,

verbose $=1$

)

optimal_params_3.fit(X_train,

y_train,

early_stopping_rounds $=10$,

eval_set $=\left[\left(\mathrm{X} \_\right.\right.$test, $\mathrm{y} \_$test $\left.)\right]$,

verbose $=$ False)

print("Gamma: ", optimal_params_3.best_params_['gamma'])

Fitting 10 folds for each of 5 candidates, totalling 50 fits

[Parallel(n_jobs=-1)]: Using backend LokyBackend with 12 concurrent workers.

[Parallel(n_jobs=-1)]: Done 26 tasks | elapsed: 3.5min 
[Parallel(n_jobs=-1)]: Done 50 out of 50 | elapsed: 5.3 min finished

Gamma: 0.003

gamma $=$ optimal_params_3.best_params_['gamma']

y_train_pred $=$ optimal_params_3.best_estimator_predict(X_train)

y_test_pred = optimal_params_3.best_estimator_.predict(X_test)

print('XGBregressor evaluating result:')

print("Train MAE: ", sklearn.metrics.mean_absolute_error(y_train, y_train_pred))

print("Train RMSE: ", np.sqrt(sklearn.metrics.mean_squared_error(y_train, y_train_pred))) print("Train R2: ", np.sqrt(sklearn.metrics.r2_score(y_train, y_train_pred)))

print("Test MAE: ", sklearn.metrics.mean_absolute_error(y_test, y_test_pred))

print("Test RMSE: ", np.sqrt(sklearn.metrics.mean_squared_error(y_test, y_test_pred)))

print("Test R2: ", np.sqrt(sklearn.metrics.r2_score(y_test, y_test_pred)))

XGBregressor evaluating result:

Train MAE: 1.1557842730348065

Train RMSE: 1.6082375631598405

Train R2: 0.6148797875288853

Test MAE: 1.1723220292261036

Test RMSE: 1.6260654683448836

Test R2: 0.5819676548322498

\# max_delta_step

param_grid_4 = \{'learning_rate':[learning_rate], 'n_estimators':[n_estimators],

'max_depth':[max_depth],

'min_child_weight':[min_child_weight], 'gamma':[gamma], 'max_delta_step':[10,11,12], \}

xgb_model $=$ XGBRegressor $($ objective $=$ 'reg:squarederror', seed=seed)

optimal_params_4 = GridSearchCV(

estimator $=x g b \_$model,

param_grid = param_grid_4,

scoring = 'r2', \#MAE

\#scoring = 'neg_mean_squared_error', \#MSE

$\mathrm{cv}=\mathrm{cv}$,

$\mathrm{n} \_$jobs $=-1$,

verbose $=1$

)

optimal_params_4.fit(X_train,

y_train,

early_stopping_rounds $=10$, 
eval_set=[(X_test, y_test $)]$,

verbose $=$ False)

print("max_delta_step: ",optimal_params_4.best_params_['max_delta_step'])

Fitting 10 folds for each of 5 candidates, totalling 50 fits

[Parallel(n_jobs=-1)]: Using backend LokyBackend with 12 concurrent workers.

[Parallel(n_jobs=-1)]: Done 26 tasks | elapsed: $3.6 \mathrm{~min}$

[Parallel(n_jobs=-1)]: Done 50 out of 50 | elapsed: $5.5 \mathrm{~min}$ finished

max_delta_step: 11

max_delta_step $=$ optimal_params_4.best_params_['max_delta_step']

y_train_pred $=$ optimal_params_4.best_estimator_.predict(X_train)

y_test_pred = optimal_params_4.best_estimator_.predict(X_test)

print('XGBregressor evaluating result:')

print("Train MAE: ", sklearn.metrics.mean_absolute_error(y_train, y_train_pred))

print("Train RMSE: ", np.sqrt(sklearn.metrics.mean_squared_error(y_train, y_train_pred)))

print("Train R2: ", np.sqrt(sklearn.metrics.r2_score(y_train, y_train_pred)))

print("Test MAE:", sklearn.metrics.mean_absolute_error(y_test, y_test_pred))

print("Test RMSE: ", np.sqrt(sklearn.metrics.mean_squared_error(y_test, y_test_pred)))

print("Test R2: ", np.sqrt(sklearn.metrics.r2_score(y_test, y_test_pred)))

XGBregressor evaluating result:

Train MAE: 1.1557842730348065

Train RMSE: 1.6082375631598405

Train R2: 0.6148797875288853

Test MAE: 1.1723220292261036

Test RMSE: 1.6260654683448836

Test R2: 0.5819676548322498

\# reg_lambda, alpha

param_grid_5 $=\{$ 'learning_rate':[learning_rate $]$,

'n_estimators':[n_estimators],

'max_depth':[max_depth],

'min_child_weight':[min_child_weight],

'gamma':[gamma],

'max_delta_step':[max_delta_step],

'reg_lambda':[1,2,3,4,5],

'reg_alpha':[1,2,3,4,5]

\}

xgb_model $=$ XGBRegressor $($ objective $=$ 'reg:squarederror', seed=seed)

optimal_params_5 = GridSearchCV( 


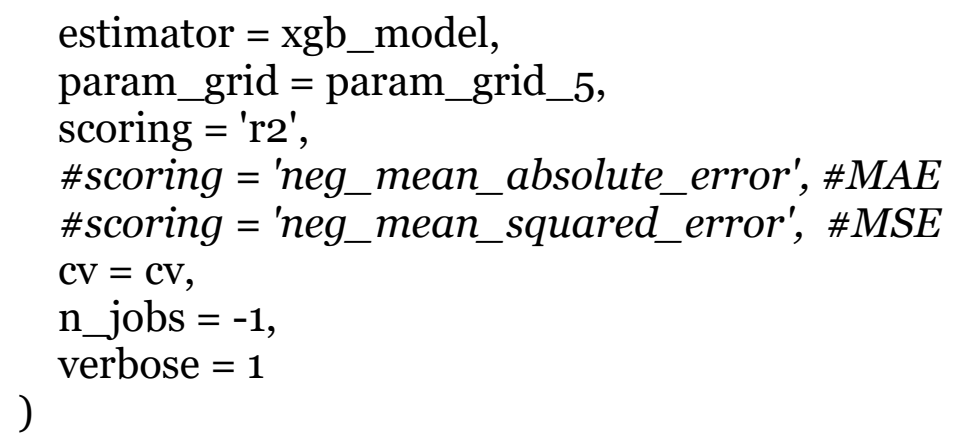

optimal_params_5.fit(X_train, y_train, early_stopping_rounds $=10$, eval_set $=\left[\left(\mathrm{X}\right.\right.$ _test, $\mathrm{y} \_$test $\left.)\right]$, verbose $=$ False)

print("Reg_lambda: ", optimal_params_5.best_params_['reg_lambda']) print("Reg_alpha: ", optimal_params_5.best_params_['reg_alpha'])

Fitting 10 folds for each of 25 candidates, totalling 250 fits

[Parallel(n_jobs=-1)]: Using backend LokyBackend with 12 concurrent workers.

[Parallel(n_jobs=-1)]: Done 26 tasks | elapsed: $3.8 \mathrm{~min}$

[Parallel(n_jobs=-1)]: Done 176 tasks | elapsed: $18.5 \mathrm{~min}$

[Parallel(n_jobs=-1)]: Done 250 out of 250 | elapsed: $25.6 \mathrm{~min}$ finished

Reg_lambda: 2

Reg_alpha: 1

reg_lambda $=$ optimal_params_5.best_params_['reg_lambda']

reg_alpha $=$ optimal_params_5.best_params_['reg_alpha']

y_train_pred $=$ optimal_params_5.best_estimator_predict(X_train)

y_test_pred = optimal_params_5.best_estimator_.predict(X_test)

print('XGBregressor evaluating result:')

print("Train MAE: ", sklearn.metrics.mean_absolute_error(y_train, y_train_pred))

print("Train RMSE:", np.sqrt(sklearn.metrics.mean_squared_error(y_train, y_train_pred)))

print("Train R2: ", np.sqrt(sklearn.metrics.r2_score(y_train, y_train_pred)))

print("Test MAE: ", sklearn.metrics.mean_absolute_error(y_test, y_test_pred))

print("Test RMSE: ", np.sqrt(sklearn.metrics.mean_squared_error(y_test, y_test_pred)))

print("Test R2: ", np.sqrt(sklearn.metrics.r2_score(y_test, y_test_pred)))

XGBregressor evaluating result:

Train MAE: 1.1564939892972832

Train RMSE: 1.6098665862703325

Train R2: 0.613853885972447

Test MAE: 1.1725238251572232

Test RMSE: 1.6260069515321633

Test R2: 0.5820085458766149 
\# subsample, colsample_bytree

param_grid_6 = \{'learning_rate':[learning_rate],

'n_estimators':[n_estimators],

'max_depth':[max_depth],

'min_child_weight':[min_child_weight],

'gamma':[gamma],

'max_delta_step':[max_delta_step],

'reg_lambda':[reg_lambda],

'reg_alpha':[reg_alpha],

'colsample_bytree':[0.5,0.7,0.9],

'subsample':[0.5,0.7,0.9]

\}

xgb_model $=$ XGBRegressor $($ objective $=$ 'reg:squarederror', seed=seed)

optimal_params_6 = GridSearchCV(

estimator $=x g b \_$model,

param_grid = param_grid_6,

scoring = 'r2',

\#scoring = 'neg_mean_absolute_error', \#MAE

\#scoring = 'neg_mean_squared_error', \#MSE

$\mathrm{cv}=\mathrm{cv}$,

$\mathrm{n} \_$jobs $=-1$,

verbose $=1$

)

optimal_params_6.fit(X_train,

y_train,

early_stopping_rounds $=10$,

eval_set $=\left[\left(X \_\right.\right.$test, $\left.\left.y \_t e s t\right)\right]$,

verbose $=$ False)

print("colsample_bytree: ", optimal_params_6.best_params_['colsample_bytree']) print("subsample: ", optimal_params_6.best_params_['subsample'])

Fitting 10 folds for each of 9 candidates, totalling 90 fits

[Parallel(n_jobs=-1)]: Using backend LokyBackend with 12 concurrent workers.

[Parallel(n_jobs=-1)]: Done 26 tasks | elapsed: $2.6 \mathrm{~min}$

[Parallel(n_jobs=-1)]: Done 90 out of 90 | elapsed: $8.2 \mathrm{~min}$ finished

colsample_bytree: 0.7

subsample: 0.7

colsample_bytree = optimal_params_6.best_params_['colsample_bytree']

subsample = optimal_params_6.best_params_['subsample'] 
y_train_pred $=$ optimal_params_6.best_estimator_.predict(X_train)

y_test_pred = optimal_params_6.best_estimator_.predict(X_test)

print('XGBregressor evaluating result:')

print("Train MAE: ", sklearn.metrics.mean_absolute_error(y_train, y_train_pred))

print("Train RMSE: ", np.sqrt(sklearn.metrics.mean_squared_error(y_train, y_train_pred)))

print("Train R2: ", np.sqrt(sklearn.metrics.r2_score(y_train, y_train_pred)))

print("Test MAE: ", sklearn.metrics.mean_absolute_error(y_test, y_test_pred))

print("Test RMSE: ", np.sqrt(sklearn.metrics.mean_squared_error(y_test, y_test_pred)))

print("Test R2: ", np.sqrt(sklearn.metrics.r2_score(y_test, y_test_pred)))

XGBregressor evaluating result:

Train MAE: 1.1572504735959093

Train RMSE: 1.608848084830042

Train R2: 0.6144956247430783

Test MAE: 1.1714188531336023

Test RMSE: 1.6218698997451828

Test R2: 0.5848885253562806

model $=$ XGBRegressor $($ booster $=$ 'gbtree', learning_rate= learning_rate,

reg_alpha=reg_alpha,

colsample_bytree=colsample_bytree,

gamma $=$ gamma,

reg_lambda= reg_lambda,

max_delta_step= max_delta_step,

max_depth=max_depth,

min_child_weight $=$ min_child_weight,

n_estimators $=$ n_estimators,

sampling_method='uniform',

subsample $=$ subsample,

seed=seed)

model.fit(X_train, y_train)

XGBRegressor(base_score $=0.5$, booster $=$ 'gbtree', colsample_bylevel $=1$,

colsample_bynode $=1$, colsample_bytree $=0.7$, gamma $=0.003$, gpu_id $=-1$,

importance_type='gain', interaction_constraints=",

learning_rate $=0.009$, max_delta_step $=11$, max_depth $=5$,

min_child_weight $=12$, missing $=$ nan, monotone_constraints $={ }^{\prime}()^{\prime}$,

n_estimators $=670, \mathrm{n} \_$jobs $=0$, num_parallel_tree $=1$, random_state $=33$,

reg_alpha $=1$, reg_lambda $=2$, sampling_method='uniform',

scale_pos_weight $=1$, seed $=33$, subsample $=0.7$, tree_method='exact', validate_parameters $=1$, verbosity $=$ None)

variables $=[$

"Age at diagnosis",

"Hispanic",

"Metro locality",

"Physical condition total","Mental health condition total",

"A1c screening","Influenza vaccination","Lipid screening", 
"SEER Northcentral","SEER Northeast","SEER South","SEER West",

"White race","Black race","Other race",

"Psyhcology visit","Primary Care visit",

"Median income",

"Urologist density","Radiation Oncologist density",

"College education","Less than highschool education",

"PCCI",

"Married","Separated/Divorced/Widowed","Unmarried","Marital status unknown",

"Low-risk prostate cancer",

"Low value care (sum)",

"Conservative management",

]

"Care fragmentation"

import shap

explainer $=$ shap.TreeExplainer $($ model $)$

shap_values = explainer.shap_values(X_train)

\# Shap values for positive class

shap.summary_plot(shap_values, X_train, max_display=10)

\# Shap values for positive class

shap.summary_plot(shap_values, X_train, max_display=10, plot_type="bar", cmap=plt.get_cmap(" cool"))

shap.dependence_plot("Physical condition total", shap_values, X_train, interaction_index = None)

shap.dependence_plot("Care fragmentation", shap_values, X_train, interaction_index = None)

shap.dependence_plot("Low value care (sum)", shap_values, X_train, interaction_index = None)

shap.dependence_plot("Conservative management", shap_values, X_train, interaction_index = None )

shap.dependence_plot("crfrg_10x", shap_values, X_train, interaction_index = "sum_lowval_12mbef ")

shap.dependence_plot("mm_pcci", shap_values, X_train, interaction_index = "sum_lowval_12mbef ")

shap.dependence_plot("mm_pcci", shap_values, X_train, interaction_index = "crfrg_10x")

shap.dependence_plot("Low value care (sum)", shap_values, X_train, interaction_index = "Physical condition total") 